Supporting Information for

\title{
Synthesis of $\beta$-Fluoro- $\alpha, \beta$-Unsaturated Amides from the Fragmentation of Morpholine 3,3,3-Trifluoropropanamide by Grignard Reagents
}

\author{
Amna T. Adam, ${ }^{\dagger}$ Frank R. Fronczek, ${ }^{\ddagger}$ and David A. Colby ${ }^{\dagger, *}$ \\ ${ }^{\dagger}$ Department of BioMolecular Sciences, University of Mississippi, University, Mississippi 38677 \\ * Department of Chemistry, Louisiana State University, Baton Rouge, Louisiana 70803
}

\section{Table of Contents:}

I. Experimental and Characterization Data.......................................S1

II. Mechanistic Experiments...........................................................S8

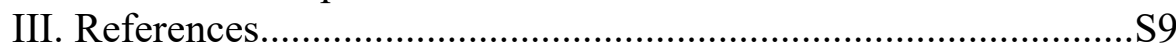

IV. X-ray Experimental and Crystallographic Data..........................S9

V. Spectral Data................................................................................. 11

\section{Experimental and Characterization Data}

General Procedures. Experiments requiring anhydrous conditions were performed under argon atmosphere and organic solvents were dried over molecular sieves. All solvents and reagents were purchased from commercial sources unless otherwise noted. Thin-layer chromatography was conducted using MilliporeSigma TLC silica gel $60 \mathrm{~F}_{254}$ plates. Preparative thin-layer chromatography was performed using Sorbent Technologies silica G prep TLC plates with UV254. Flash chromatography was conducted using SiliCycle Siliaflash silica gel P60 $(40-63 \mu \mathrm{m}) 60 \AA$. Melting points were taken on an OptiMelt apparatus from Stanford Research Systems and are not corrected. NMR spectra were recorded on a Bruker Topspin Avance III HD $500 \mathrm{MHz}$ spectrometer equipped with prodigy cryoprobe or a Bruker Avance III HD $400 \mathrm{MHz}$ spectrometer. The residual solvent peaks were used as an internal standard for ${ }^{1} \mathrm{H}$ and ${ }^{13} \mathrm{C}$ NMR spectra whereas trifluorotoluene was used as an added internal standard for ${ }^{19} \mathrm{~F}$ NMR spectra. Mass spectrometry were acquired by the Department of Chemistry at the University of Mississippi using SYNAPT HD Mass Spectrometer from Waters. Infrared spectra were recorded on Agilent Technologies Cary 630 FTIR. X-ray crystallography was performed at the X-ray Facility at the Louisiana State University. Elemental analyses were carried out by Midwest Microlab.

HO $\mathrm{CF}_{3} \frac{\mathrm{EDCl}, \mathrm{Et} \mathrm{t}_{3} \mathrm{~N}, \mathrm{HOBT}}{\begin{array}{c}\text { morpholine, THF/DMF, } \Delta, \\ \text { quant. }\end{array}}$

Morpholine 3,3,3-trifluoropropanamide (1). To a solution of 3,3,3-trifluoropropionic acid in THF/DMF $(9: 1,150 \mathrm{~mL})$ at $\mathrm{rt}$ was added morpholine $(1.5 \mathrm{~mL}, 17 \mathrm{mmol})$, triethylamine $(1.8 \mathrm{~mL}$, $12 \mathrm{mmol}), 1$-hydroxybenzotriazole hydrate $(1.76 \mathrm{~g}, 13.0 \mathrm{mmol})$, and $N$-(3-dimethylaminopropyl)$\mathrm{N}$-ethylcarbodiimide $\mathrm{HCl}(2.5 \mathrm{~g}, 13 \mathrm{mmol})$. The reaction mixture was heated for $24 \mathrm{~h}$ in an oil bath 
at $65^{\circ} \mathrm{C}$. Next, the reaction mixture was cooled to rt, diluted with EtOAc $(50 \mathrm{~mL})$, quenched with a saturated aqueous $\mathrm{NH}_{4} \mathrm{Cl}(30 \mathrm{~mL})$, and extracted with EtOAc $(3 \times 25 \mathrm{~mL})$. The organics were washed with $1.0 \mathrm{~N} \mathrm{HCl}$ solution $(30 \mathrm{~mL})$, saturated aqueous $\mathrm{NaHCO}_{3}(30 \mathrm{~mL})$, saturated aqueous $\mathrm{NaCl}(50 \mathrm{~mL})$, dried over $\mathrm{Na}_{2} \mathrm{SO}_{4}$, and concentrated under reduced pressure. The crude reaction mixture was purified by $\mathrm{SiO}_{2}$ flash chromatography $(10 \rightarrow 30 \%$ EtOAc in hexanes) to yield the title compound 1 as a colorless solid (2.24 g, quant.). Recrystallization from a 1:1 solution of hexanes and cyclohexanes (by slow evaporation) provided a crystalline solid suitable for X-ray structure analysis: $\mathrm{mp} 76{ }^{\circ} \mathrm{C} ;{ }^{1} \mathrm{H} \mathrm{NMR}\left(500 \mathrm{MHz}, \mathrm{CDCl}_{3}\right) \delta 3.68(\mathrm{~m}, 6 \mathrm{H}), 3.47(\mathrm{t}, J=4.8 \mathrm{~Hz}, 2 \mathrm{H}), 3.23(\mathrm{q}$, $J=10.1 \mathrm{~Hz}, 2 \mathrm{H}) ;{ }^{13} \mathrm{C} \mathrm{NMR}\left(125 \mathrm{MHz}, \mathrm{CDCl}_{3}\right) \delta 161.6\left(\mathrm{q}, J_{\mathrm{CF}}=3.3 \mathrm{~Hz}, 1 \mathrm{C}\right), 124.0\left(\mathrm{q}, J_{\mathrm{CF}}=275.3\right.$ $\mathrm{Hz}, 1 \mathrm{C}), 66.7,66.4,46.8,42.3,38.0\left(\mathrm{q}, J_{\mathrm{CF}}=29.0 \mathrm{~Hz}, 1 \mathrm{C}\right) ;{ }^{19} \mathrm{~F} \mathrm{NMR}\left(376 \mathrm{MHz}, \mathrm{CDCl}_{3}\right) \delta-62.41$ (t, $J_{\mathrm{HF}}=10.0 \mathrm{~Hz}, 3 \mathrm{~F}$ ); IR (film) $v_{\max } 2862,1653,1439,1094 \mathrm{~cm}^{-1}$; HRMS (ESI-TOF) $\mathrm{m} / z$ calcd for $\mathrm{C}_{7} \mathrm{H}_{10} \mathrm{~F}_{3} \mathrm{NO}_{2} \mathrm{Cs}[\mathrm{M}+\mathrm{Cs}]^{+} 329.9718$, found 329.9741; Anal. Calcd for $\mathrm{C}_{7} \mathrm{H}_{10} \mathrm{~F}_{3} \mathrm{NO}_{2} \cdot 0.2 \mathrm{C}_{6} \mathrm{H}_{6}$ : $\mathrm{C}$, 46.29; H, 5.46; N, 6.58. Found: C, 46.62; H, 5.46; N, 6.79.

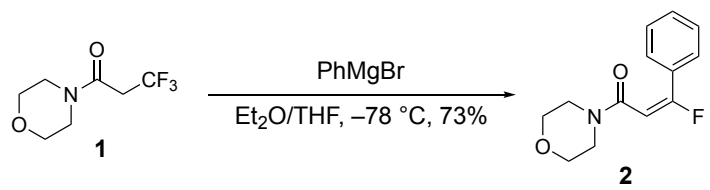

(E)-3-Fluoro-1-morpholino-3-phenylprop-2-en-1-one (2). A solution of 1 (100 mg, $0.51 \mathrm{mmol})$ in $\mathrm{THF} / \mathrm{Et}_{2} \mathrm{O}(1: 1,2 \mathrm{~mL})$ was cooled to $-78{ }^{\circ} \mathrm{C}$, treated with a solution of phenylmagnesium bromide (1.0 mL, 1.0 M in THF), and stirred for $4 \mathrm{~h}$. The reaction mixture was quenched with 0.2 $\mathrm{N} \mathrm{HCl}$ solution $(2 \mathrm{~mL})$ and extracted with $\mathrm{CH}_{2} \mathrm{Cl}_{2}(3 \times 5 \mathrm{~mL})$. The combined organics were washed with saturated aqueous $\mathrm{NaCl}(10 \mathrm{~mL})$, dried over $\mathrm{Na}_{2} \mathrm{SO}_{4}$, and concentrated under reduced pressure. $\mathrm{SiO}_{2}$ flash chromatography $(10 \rightarrow 90 \%$ EtOAc in hexanes $)$ afforded the title compound 2 as a pale yellow oil $\left(88 \mathrm{mg}, 73 \%\right.$ yield): ${ }^{1} \mathrm{H} \mathrm{NMR}\left(400 \mathrm{MHz}, \mathrm{CDCl}_{3}\right) \delta 7.56(\mathrm{~d}, J=6.8 \mathrm{~Hz}, 2 \mathrm{H})$, $7.41(\mathrm{~d}, J=7.5 \mathrm{~Hz}, 2 \mathrm{H}), 7.40(\mathrm{~m}, 1 \mathrm{H}), 5.86(\mathrm{~d}, J=19.3 \mathrm{~Hz}, 1 \mathrm{H}), 3.61(\mathrm{~d}, J=5.3 \mathrm{~Hz}, 4 \mathrm{H}), 3.34(\mathrm{~d}$, $J=5.2 \mathrm{~Hz}, 2 \mathrm{H}), 3.30(\mathrm{~d}, J=5.0 \mathrm{~Hz}, 2 \mathrm{H}) ;{ }^{13} \mathrm{C} \mathrm{NMR}\left(125 \mathrm{MHz}, \mathrm{CDCl}_{3}\right) \delta 164.4\left(\mathrm{~d}, J_{\mathrm{CF}}=18.6 \mathrm{~Hz}\right.$, $1 \mathrm{C}), 161.7\left(\mathrm{~d}, J_{\mathrm{CF}}=257.8 \mathrm{~Hz}, 1 \mathrm{C}\right), 130.7,130.4\left(\mathrm{~d}, J_{\mathrm{CF}}=28.2 \mathrm{~Hz}, 1 \mathrm{C}\right), 128.5(2 \mathrm{C}), 127.2\left(\mathrm{~d}, J_{\mathrm{CF}}\right.$ $=6.0 \mathrm{~Hz}, 2 \mathrm{C}), 101.7\left(\mathrm{~d}, J_{\mathrm{CF}}=27.9 \mathrm{~Hz}, 1 \mathrm{C}\right), 66.3(2 \mathrm{C}), 46.8,41.8 ;{ }^{19} \mathrm{~F} \mathrm{NMR}\left(376 \mathrm{MHz}, \mathrm{CDCl}_{3}\right) \delta$ $-96.7\left(\mathrm{~d}, J_{\mathrm{HF}}=19.3 \mathrm{~Hz}, 1 \mathrm{~F}\right)$; IR (film) $v_{\max } 2857,1627,1433,1109 \mathrm{~cm}^{-1}$; HRMS (ESI-TOF) $\mathrm{m} / z$ calcd for $\mathrm{C}_{13} \mathrm{H}_{14} \mathrm{FNO}_{2} \mathrm{Cs}[\mathrm{M}+\mathrm{Cs}]^{+}$368.0063, found 368.0076 .

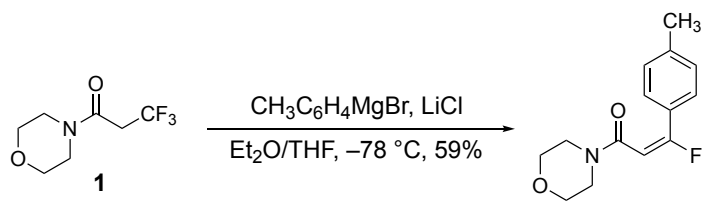

3

(E)-3-Fluoro-1-morpholino-3-(p-tolyl)prop-2-en-1-one (3). A solution of 1 (100 $\mathrm{mg}, 0.51$ $\mathrm{mmol})$ and $\mathrm{LiCl}(43.0 \mathrm{mg}, 1.0 \mathrm{mmol})$ in $\mathrm{THF} / \mathrm{Et}_{2} \mathrm{O}(1: 1,8 \mathrm{~mL})$ was cooled to $-78{ }^{\circ} \mathrm{C}$, treated with

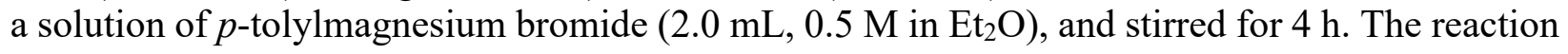
mixture was quenched with $0.2 \mathrm{~N} \mathrm{HCl}$ solution $(2 \mathrm{~mL})$ and extracted with $\mathrm{CH}_{2} \mathrm{Cl}_{2}(3 \times 5 \mathrm{~mL})$. The combined organics were washed with saturated aqueous $\mathrm{NaCl}(10 \mathrm{~mL})$, dried over $\mathrm{Na}_{2} \mathrm{SO}_{4}$, and concentrated under reduced pressure. $\mathrm{SiO}_{2}$ flash chromatography $(20 \rightarrow 80 \%$ EtOAc in hexanes) afforded the title compound 3 as a pale yellow oil $(75 \mathrm{mg}, 59 \%):{ }^{1} \mathrm{H} \mathrm{NMR}\left(400 \mathrm{MHz}, \mathrm{CDCl}_{3}\right) \delta$ $7.45(\mathrm{~d}, J=8.3 \mathrm{~Hz}, 2 \mathrm{H}), 7.20(\mathrm{~d}, J=8.4 \mathrm{~Hz}, 2 \mathrm{H}), 5.81(\mathrm{~d}, J=19.3 \mathrm{~Hz}, 1 \mathrm{H}), 3.62$ (app d, $J=2.6$ $\mathrm{Hz}, 4 \mathrm{H}), 3.34(\operatorname{app} \mathrm{d}, J=4.0 \mathrm{~Hz}, 4 \mathrm{H}), 2.38(\mathrm{~s}, 3 \mathrm{H}) ;{ }^{13} \mathrm{C} \mathrm{NMR}\left(100 \mathrm{MHz}, \mathrm{CDCl}_{3}\right) \delta 164.6\left(\mathrm{~d}, J_{\mathrm{CF}}\right.$ 
$=18.7 \mathrm{~Hz}, 1 \mathrm{C}), 162.0\left(\mathrm{~d}, J_{\mathrm{CF}}=257.5 \mathrm{~Hz}, 1 \mathrm{C}\right), 141.1,129.2(2 \mathrm{C}), 127.5\left(\mathrm{~d}, J_{\mathrm{CF}}=28.3 \mathrm{~Hz}, 1 \mathrm{C}\right)$, $127.1\left(\mathrm{~d}, J_{\mathrm{CF}}=5.9 \mathrm{~Hz}, 2 \mathrm{C}\right), 100.9\left(\mathrm{~d}, J_{\mathrm{CF}}=28.3 \mathrm{~Hz}, 1 \mathrm{C}\right), 66.3(2 \mathrm{C}), 46.8,41.8,21.4 ;{ }^{19} \mathrm{~F} \mathrm{NMR}$ $\left(376 \mathrm{MHz}, \mathrm{CDCl}_{3}\right) \delta-96.3\left(\mathrm{~d}, J_{\mathrm{HF}}=19.2 \mathrm{~Hz}, 1 \mathrm{~F}\right)$; IR (film) $v_{\max } 2855,1627,1431,1113 \mathrm{~cm}^{-1}$; HRMS (ESI-TOF) $m / z$ calcd for $\mathrm{C}_{14} \mathrm{H}_{17} \mathrm{FNO}_{2}[\mathrm{M}+\mathrm{H}]^{+} 250.1243$, found 250.1273 .

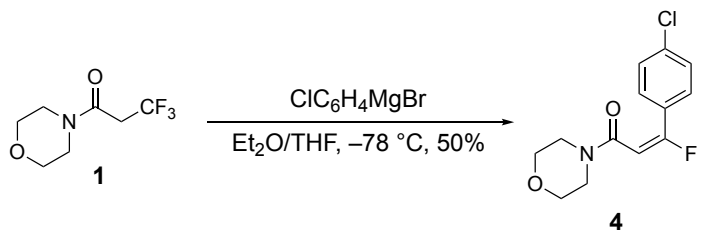

(E)-3-(4-chlorophenyl)-3-fluoro-1-morpholinoprop-2-en-1-one (4). A solution of 1 (100 mg, $0.51 \mathrm{mmol})$ in $\mathrm{THF} / \mathrm{Et}_{2} \mathrm{O}(1: 1,2 \mathrm{~mL})$ was cooled to $-78{ }^{\circ} \mathrm{C}$, treated with a solution of 4 chlorophenylmagnesium bromide $\left(1.0 \mathrm{~mL}, 1.0 \mathrm{M} \mathrm{in} \mathrm{Et}_{2} \mathrm{O}\right)$, and stirred for $4 \mathrm{~h}$. The reaction mixture was quenched with $0.2 \mathrm{~N} \mathrm{HCl}$ solution $(2 \mathrm{~mL})$ and extracted with $\mathrm{CH}_{2} \mathrm{Cl}_{2}(3 \times 5 \mathrm{~mL})$. The combined organics were washed with saturated aqueous $\mathrm{NaCl}(10 \mathrm{~mL})$, dried over $\mathrm{Na}_{2} \mathrm{SO}_{4}$, and concentrated under reduced pressure. $\mathrm{SiO}_{2}$ flash chromatography in $(5 \rightarrow 70 \%$ EtOAc in hexanes) afforded the title compound 4 as a colorless oil (69 mg, 50\%): ${ }^{1} \mathrm{H} \mathrm{NMR}\left(400 \mathrm{MHz}, \mathrm{CDCl}_{3}\right) \delta 7.52$ $(\mathrm{d}, J=8.6 \mathrm{~Hz}, 2 \mathrm{H}), 7.36(\mathrm{~d}, J=8.4 \mathrm{~Hz}, 2 \mathrm{H}), 5.90(\mathrm{~d}, J=19.9 \mathrm{~Hz}, 1 \mathrm{H}), 3.61(\mathrm{~m}, 4 \mathrm{H}), 3.39$ (app d, $J=6.0 \mathrm{~Hz}, 4 \mathrm{H}) ;{ }^{13} \mathrm{C} \mathrm{NMR}\left(100 \mathrm{MHz}, \mathrm{CDCl}_{3}\right) \delta 163.9\left(\mathrm{~d}, J_{\mathrm{CF}}=18.5 \mathrm{~Hz}, 1 \mathrm{C}\right), 161.1\left(\mathrm{~d}, J_{\mathrm{CF}}=257.0\right.$ $\mathrm{Hz}, 1 \mathrm{C}), 136.8,128.7\left(\mathrm{~d}, J_{\mathrm{CF}}=28.7 \mathrm{~Hz}, 1 \mathrm{C}\right), 128.7(2 \mathrm{C}), 128.6\left(\mathrm{~d}, J_{\mathrm{CF}}=6.2 \mathrm{~Hz}, 2 \mathrm{C}\right), 102.1\left(\mathrm{~d}, J_{\mathrm{CF}}\right.$ $=28.0 \mathrm{~Hz}, 1 \mathrm{C}), 66.4(2 \mathrm{C}), 46.7,41.8 ;{ }^{19} \mathrm{~F} \mathrm{NMR}\left(376 \mathrm{MHz}, \mathrm{CDCl}_{3}\right) \delta-96.1\left(\mathrm{~d}, J_{\mathrm{HF}}=19.9 \mathrm{~Hz}, 1 \mathrm{~F}\right)$; IR (film) $v_{\max } 2855,1623,1431,1113 \mathrm{~cm}^{-1}$; HRMS (ESI-TOF) $\mathrm{m} / z$ calcd for $\mathrm{C}_{13} \mathrm{H}_{14} \mathrm{ClFNO}_{2}$ $[\mathrm{M}+\mathrm{H}]^{+} 270.0697$, found 270.0699 .

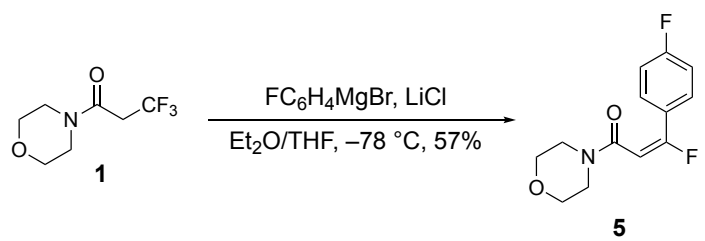

(E)-3-fluoro-3-(4-fluorophenyl)-1-morpholinoprop-2-en-1-one (5). A solution of 1 (100 mg, $0.51 \mathrm{mmol})$ and $\mathrm{LiCl}(43.0 \mathrm{mg}, 1.0 \mathrm{mmol})$ in $\mathrm{THF} / \mathrm{Et}_{2} \mathrm{O}(1: 1,8 \mathrm{~mL})$ was cooled to $-78{ }^{\circ} \mathrm{C}$, treated with a solution of 4-fluorophenylmagnesium bromide $\left(0.5 \mathrm{~mL}, 2.0 \mathrm{M}\right.$ in $\left.\mathrm{Et}_{2} \mathrm{O}\right)$, and stirred for $4 \mathrm{~h}$. The reaction mixture was quenched with $0.2 \mathrm{~N} \mathrm{HCl}$ solution $(8 \mathrm{~mL})$ and extracted with $\mathrm{CH}_{2} \mathrm{Cl}_{2}(3$ $\times 5 \mathrm{~mL})$. The combined organics were washed with saturated aqueous $\mathrm{NaCl}(10 \mathrm{~mL})$, dried over $\mathrm{Na}_{2} \mathrm{SO}_{4}$, and concentrated under reduced pressure. $\mathrm{SiO}_{2}$ flash chromatography $(20 \rightarrow 80 \%$ EtOAc in hexanes) afforded the title compound $\mathbf{5}$ as an orange-yellow oil (74 $\mathrm{mg}, 57 \%):{ }^{1} \mathrm{H}$ NMR (500 $\left.\mathrm{MHz} \mathrm{CDCl}_{3}\right) \delta 7.59(\mathrm{dd}, J=8.7,5.3 \mathrm{~Hz}, 2 \mathrm{H}), 7.08(\mathrm{t}, J=8.6 \mathrm{~Hz}, 2 \mathrm{H}), 5.87(\mathrm{~d}, J=19.8 \mathrm{~Hz}, 1 \mathrm{H})$, $3.62(\mathrm{~m}, 4 \mathrm{H}), 3.39(\mathrm{~m}, 4 \mathrm{H}) ;{ }^{13} \mathrm{C} \mathrm{NMR}\left(125 \mathrm{MHz}, \mathrm{CDCl}_{3}\right) \delta 164.1\left(\mathrm{~d}, J_{\mathrm{CF}}=18.6 \mathrm{~Hz}, 1 \mathrm{C}\right), 163.9$ $\left(\mathrm{d}, J_{\mathrm{CF}}=250.8 \mathrm{~Hz}, 1 \mathrm{C}\right), 161.2\left(\mathrm{~d}, J_{\mathrm{CF}}=257.2 \mathrm{~Hz}, 1 \mathrm{C}\right), 129.6\left(\mathrm{dd}, J_{\mathrm{CF}}=8.7,6.2 \mathrm{~Hz}, 2 \mathrm{C}\right), 126.5$ $\left(\mathrm{dd}, J_{\mathrm{CF}}=28.8,3.4 \mathrm{~Hz}, 1 \mathrm{C}\right), 115.7\left(\mathrm{~d}, J_{\mathrm{CF}}=22.0 \mathrm{~Hz}, 2 \mathrm{C}\right), 101.5\left(\mathrm{~d}, J_{\mathrm{CF}}=28.2 \mathrm{~Hz}, 1 \mathrm{C}\right), 66.4(2 \mathrm{C})$, 46.8, 41.9; ${ }^{19} \mathrm{~F}$ NMR $\left(471 \mathrm{MHz}, \mathrm{CDCl}_{3}\right) \delta-95.7\left(\mathrm{~d}, J_{\mathrm{HF}}=19.8 \mathrm{~Hz}, 1 \mathrm{~F}\right),-108.3\left(\mathrm{ddd}, J_{\mathrm{HF}}=13.6\right.$, 8.5, $5.3 \mathrm{~Hz}, 1 \mathrm{~F}$ ); IR (film) $v_{\max } 2855,1625,1508,1228,1113 \mathrm{~cm}^{-1}$; HRMS (ESI-TOF) $\mathrm{m} / z$ calcd for $\mathrm{C}_{13} \mathrm{H}_{14} \mathrm{~F}_{2} \mathrm{NO}_{2}[\mathrm{M}+\mathrm{H}]^{+}$254.0993, found 254.0978. 


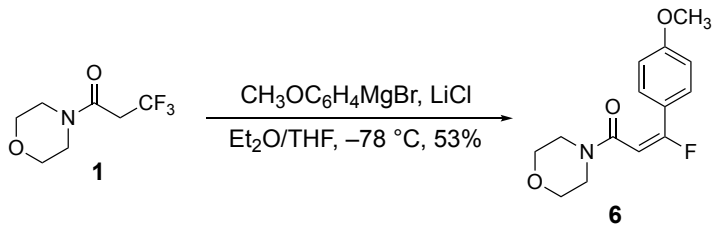

(E)-3-fluoro-3-(4-methoxyphenyl)-1-morpholinoprop-2-en-1-one (6). A solution of 1 (100 mg, $0.51 \mathrm{mmol})$ and $\mathrm{LiCl}(65 \mathrm{mg}, 1.5 \mathrm{mmol})$ in $\mathrm{THF} / \mathrm{Et}_{2} \mathrm{O}(1: 1,6 \mathrm{~mL})$ was cooled to $-78{ }^{\circ} \mathrm{C}$, treated with a solution of 4-methoxyphenylmagnesium bromide (1.1 mL, 1.0 M in THF), and stirred for $4 \mathrm{~h}$. The reaction mixture was quenched with $0.2 \mathrm{~N} \mathrm{HCl}$ solution $(6 \mathrm{~mL})$ and extracted with $\mathrm{CH}_{2} \mathrm{Cl}_{2}$ $(3 \times 5 \mathrm{~mL})$. The combined organics were washed with saturated aqueous $\mathrm{NaCl}(10 \mathrm{~mL})$, dried over $\mathrm{Na}_{2} \mathrm{SO}_{4}$, and concentrated under reduced pressure. $\mathrm{SiO}_{2}$ flash chromatography $(20 \rightarrow 80 \%$ EtOAc in hexanes) afforded the title compound 6 as a yellow oil $(72 \mathrm{mg}, 53 \%):{ }^{1} \mathrm{H} \mathrm{NMR} \mathrm{(500} \mathrm{MHz,}$ $\left.\mathrm{CDCl}_{3}\right) \delta 7.51(\mathrm{~d}, J=8.7 \mathrm{~Hz}, 2 \mathrm{H}), 6.89(\mathrm{~d}, J=8.6 \mathrm{~Hz}, 2 \mathrm{H}), 5.76(\mathrm{~d}, J=19.5 \mathrm{~Hz}, 1 \mathrm{H}), 3.82(\mathrm{~s}, 3 \mathrm{H})$, $3.61(\mathrm{~m}, 4 \mathrm{H}), 3.36(\mathrm{~m}, 4 \mathrm{H}) ;{ }^{13} \mathrm{C} \mathrm{NMR}\left(125 \mathrm{MHz}, \mathrm{CDCl}_{3}\right) \delta 164.7\left(\mathrm{~d}, J_{\mathrm{CF}}=19.0 \mathrm{~Hz}, 1 \mathrm{C}\right), 162.1$ $\left(\mathrm{d}, J_{\mathrm{CF}}=255.1 \mathrm{~Hz}, 1 \mathrm{C}\right), 161.4,128.9\left(\mathrm{~d}, J_{\mathrm{CF}}=6.3 \mathrm{~Hz}, 2 \mathrm{C}\right), 122.7\left(\mathrm{~d}, J_{\mathrm{CF}}=28.8 \mathrm{~Hz}, 1 \mathrm{C}\right), 113.8$ (2C), $99.9\left(\mathrm{~d}, J_{\mathrm{CF}}=29.0 \mathrm{~Hz}, 1 \mathrm{C}\right), 66.4(2 \mathrm{C}), 55.3,46.8,41.8 ;{ }^{19} \mathrm{~F}$ NMR $\left(376 \mathrm{MHz}, \mathrm{CDCl}_{3}\right) \delta-$ $96.2\left(\mathrm{~d}, J_{\mathrm{HF}}=19.5 \mathrm{~Hz}, 1 \mathrm{~F}\right)$; IR (film) $v_{\max } 2853,1604,1429,1237,1111 \mathrm{~cm}^{-1}$; HRMS (ESI-TOF) $m / z$ calcd for $\mathrm{C}_{14} \mathrm{H}_{16} \mathrm{FNO}_{3} \mathrm{Cs}[\mathrm{M}+\mathrm{Cs}]^{+} 398.0169$, found 398.0168 .

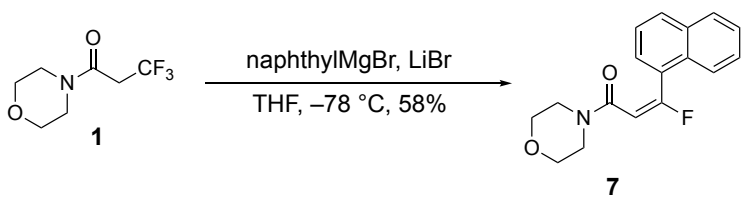

(E)-3-fluoro-1-morpholino-3-(naphthalen-1-yl)prop-2-en-1-one (7). A solution of $\mathrm{LiBr}$ (310 $\mathrm{mg}, 3.57 \mathrm{mmol})$ in THF $(24 \mathrm{~mL})$ was treated with a solution of naphthylmagnesium bromide $(21.4$ $\mathrm{mL}, 0.5 \mathrm{M}$ in MeTHF) and stirred for $1 \mathrm{~h}$ at $\mathrm{rt}$. Next, the reaction mixture was cooled to $-78{ }^{\circ} \mathrm{C}$ and a solution of $1(352 \mathrm{mg}, 1.79 \mathrm{mmol})$ in THF $(10 \mathrm{~mL})$ was added dropwise. The mixture was stirred for $4 \mathrm{~h}$ at $-78{ }^{\circ} \mathrm{C}$ and then quenched with $1 \mathrm{~N} \mathrm{HCl}$ solution $(10 \mathrm{~mL})$. The resultant mixture was diluted with EtOAc $(15 \mathrm{~mL})$ and extracted with EtOAc $(3 \times 25 \mathrm{~mL})$. The combined organics were dried over $\mathrm{Na}_{2} \mathrm{SO}_{4}$ and concentrated under reduced pressure. Recrystallization from a solution of hexanes/EtOAc (8:2, by slow evaporation) followed by $\mathrm{SiO}_{2}$ flash chromatography $(8: 2 \rightarrow 7: 3$ hexanes/EtOAc) afforded the title compound 7 as a colorless solid $(297 \mathrm{mg}, 58 \%): \mathrm{mp}$ $139-141{ }^{\circ} \mathrm{C} ;{ }^{1} \mathrm{H}$ NMR $\left(400 \mathrm{MHz}, \mathrm{CDCl}_{3}\right) \delta 8.01(\mathrm{dd}, J=8.0,2.8 \mathrm{~Hz}, 1 \mathrm{H}), 7.97(\mathrm{~d}, J=8.7 \mathrm{~Hz}$, $1 \mathrm{H}), 7.90(\mathrm{~d}, J=8.5 \mathrm{~Hz}, 1 \mathrm{H}), 7.65(\mathrm{~d}, J=7.1 \mathrm{~Hz}, 1 \mathrm{H}), 7.56(\mathrm{~m}, 2 \mathrm{H}), 7.49(\mathrm{t}, J=7.7 \mathrm{~Hz}, 1 \mathrm{H}), 6.18$ $(\mathrm{d}, J=16.7 \mathrm{~Hz}, 1 \mathrm{H}), 3.42(\mathrm{~m}, 2 \mathrm{H}), 3.33(\mathrm{~m}, 2 \mathrm{H}), 3.23(\mathrm{~m}, 2 \mathrm{H}), 2.83(\mathrm{~m}, 2 \mathrm{H}) ;{ }^{13} \mathrm{C} \mathrm{NMR}(125 \mathrm{MHz}$, $\left.\mathrm{CDCl}_{3}\right) \delta 163.9\left(\mathrm{~d}, J_{\mathrm{CF}}=17.4 \mathrm{~Hz}, 1 \mathrm{C}\right), 163.5\left(\mathrm{~d}, J_{\mathrm{CF}}=263.0 \mathrm{~Hz}, 1 \mathrm{C}\right), 133.4\left(\mathrm{~d}, J_{\mathrm{CF}}=1.3 \mathrm{~Hz}, 1 \mathrm{C}\right)$, $131.4\left(\mathrm{~d}, J_{\mathrm{CF}}=2.0 \mathrm{~Hz}, 1 \mathrm{C}\right), 130.3\left(\mathrm{~d}, J_{\mathrm{CF}}=1.1 \mathrm{~Hz}, 1 \mathrm{C}\right), 128.8\left(\mathrm{~d}, J_{\mathrm{CF}}=4.7 \mathrm{~Hz}, 1 \mathrm{C}\right), 128.7,128.0$ $\left(\mathrm{d}, J_{\mathrm{CF}}=24.9 \mathrm{~Hz}, 1 \mathrm{C}\right), 127.3\left(\mathrm{~d}, J_{\mathrm{CF}}=1.2 \mathrm{~Hz}, 1 \mathrm{C}\right), 126.5,124.9,124.6\left(\mathrm{~d}, J_{\mathrm{CF}}=3.3 \mathrm{~Hz}, 1 \mathrm{C}\right), 104.9$ $\left(\mathrm{d}, J_{\mathrm{CF}}=26.9 \mathrm{~Hz}, 1 \mathrm{C}\right), 66.5\left(\mathrm{~d}, J_{\mathrm{CF}}=30.4 \mathrm{~Hz}, 1 \mathrm{C}\right), 66.1\left(\mathrm{~d}, J_{\mathrm{CF}}=38.7 \mathrm{~Hz}, 1 \mathrm{C}\right), 46.9,41.8 ;{ }^{19} \mathrm{~F}$ NMR (471 MHz, $\left.\mathrm{CDCl}_{3}\right) \delta-82.9\left(\mathrm{~d}, J_{\mathrm{HF}}=16.9 \mathrm{~Hz}, 1 \mathrm{~F}\right)$; IR (film) $v_{\max } 3010,1670,1618,1437$, $1113 \mathrm{~cm}^{-1}$; HRMS (ESI-TOF) $m / z$ calcd for $\mathrm{C}_{17} \mathrm{H}_{17} \mathrm{FNO}_{2}[\mathrm{M}+\mathrm{H}]^{+} 286.1243$, found 286.1244.

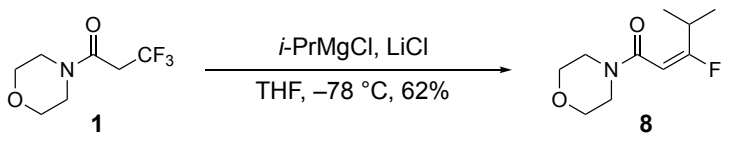


(E)-3-fluoro-4-methyl-1-morpholinopent-2-en-1-one (8). A solution of isopropylmagnesium chloride (2.2 mL, 2.0 M in THF) and $\mathrm{LiCl}(184 \mathrm{mg}, 4.34 \mathrm{mmol})$ in THF (36 mL) was stirred at $\mathrm{rt}$ for $1 \mathrm{~h}$, cooled to $-78{ }^{\circ} \mathrm{C}$, treated with a solution of 1 (428 mg, $\left.2.17 \mathrm{mmol}\right)$ in THF (7 mL), and stirred for $4 \mathrm{~h}$. The reaction mixture was quenched with $1.0 \mathrm{~N} \mathrm{HCl}$ solution $(20 \mathrm{~mL})$ and extracted with $\mathrm{CH}_{2} \mathrm{Cl}_{2}(3 \times 25 \mathrm{~mL})$. The combined organics were washed with saturated aqueous $\mathrm{NaCl}(30$ $\mathrm{mL}$ ), dried over $\mathrm{Na}_{2} \mathrm{SO}_{4}$, and concentrated under reduced pressure. $\mathrm{SiO}_{2}$ flash chromatography $(20 \rightarrow 60 \%$ EtOAc in hexanes) afforded the title compound 8 as a colorless oil $(272.4 \mathrm{mg}, 62 \%)$ : ${ }^{1} \mathrm{H}$ NMR $\left(500 \mathrm{MHz}, \mathrm{CDCl}_{3}\right) ; \delta 5.67(\mathrm{~d}, J=20.9 \mathrm{~Hz}, 1 \mathrm{H}), 3.70-3.58(\mathrm{~m}, 6 \mathrm{H}), 3.55(\mathrm{~m}, 1 \mathrm{H}), 3.48$ $(\mathrm{m}, 2 \mathrm{H}), 1.15(\mathrm{~d}, J=6.9 \mathrm{~Hz}, 6 \mathrm{H}) ;{ }^{13} \mathrm{C} \mathrm{NMR}\left(125 \mathrm{MHz}, \mathrm{CDCl}_{3}\right) \delta 177.0\left(\mathrm{~d}, J_{\mathrm{CF}}=272.9 \mathrm{~Hz}, 1 \mathrm{C}\right)$, $164.6\left(\mathrm{~d}, J_{\mathrm{CF}}=21.5 \mathrm{~Hz}, 1 \mathrm{C}\right), 98.1\left(\mathrm{~d}, J_{\mathrm{CF}}=27.5 \mathrm{~Hz}, 1 \mathrm{C}\right), 66.8,66.6,46.6,41.9,28.7\left(\mathrm{~d}, J_{\mathrm{CF}}=23.1\right.$ $\mathrm{Hz}, 1 \mathrm{C}), 18.8(2 \mathrm{C}) ;{ }^{19} \mathrm{~F}$ NMR $\left(471 \mathrm{MHz}, \mathrm{CDCl}_{3}\right) \delta-98.2\left(\mathrm{dd}, J_{\mathrm{HF}}=34.0,21.0 \mathrm{~Hz}, 1 \mathrm{~F}\right)$; IR (film) $v_{\max } 2857,1675,1618,1429,1113 \mathrm{~cm}^{-1}$; HRMS (ESI-TOF) $m / z$ calcd for $\mathrm{C}_{10} \mathrm{H}_{16} \mathrm{FNO}_{2} \mathrm{Cs}[\mathrm{M}+\mathrm{Cs}]^{+}$ 334.0220 , found 334.0236 .

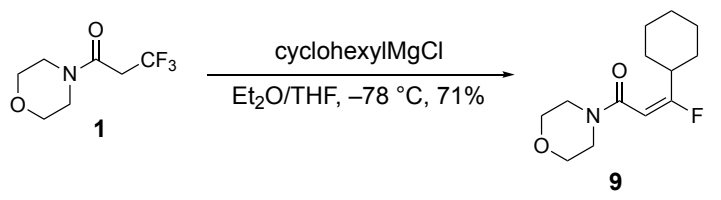

(E)-3-cyclohexyl-3-fluoro-1-morpholinoprop-2-en-1-one (9). A solution of 1 (100 mg, 0.51 $\mathrm{mmol})$ in $\mathrm{THF} / \mathrm{Et}_{2} \mathrm{O}(1: 1,3 \mathrm{~mL})$ was cooled to $-78{ }^{\circ} \mathrm{C}$, treated with a solution of cyclohexylmagnesium chloride $(0.92 \mathrm{~mL}, 1.3 \mathrm{M}$ in 1:1 THF/toluene), and stirred for $4 \mathrm{~h}$. The reaction mixture was quenched with $0.2 \mathrm{~N} \mathrm{HCl}$ solution $(3 \mathrm{~mL})$ and extracted with $\mathrm{CH}_{2} \mathrm{Cl}_{2}(3 \times 5$ $\mathrm{mL})$. The combined organics were washed with saturated aqueous $\mathrm{NaCl}(10 \mathrm{~mL})$, dried over $\mathrm{Na}_{2} \mathrm{SO}_{4}$, and concentrated under reduced pressure. $\mathrm{SiO}_{2}$ flash chromatography $(20 \rightarrow 80 \%$ EtOAc in hexanes) afforded the title compound 9 as a colorless oil $(87 \mathrm{mg}, 71 \%):{ }^{1} \mathrm{H} \mathrm{NMR}(500 \mathrm{MHz}$, $\left.\mathrm{CDCl}_{3}\right) \delta 5.65(\mathrm{~d}, J=21.2 \mathrm{~Hz}, 1 \mathrm{H}), 3.77-3.55(\mathrm{~m}, 8 \mathrm{H}), 3.20(\mathrm{dt}, J=33.2,11.9 \mathrm{~Hz}, 1 \mathrm{H}), 1.76-1.63$ $(\mathrm{m}, 5 \mathrm{H}), 1.43(\mathrm{q}, J=12.4 \mathrm{~Hz}, 2 \mathrm{H}), 1.31(\mathrm{q}, J=12.9 \mathrm{~Hz}, 2 \mathrm{H}), 1.17(\mathrm{~m}, 1 \mathrm{H}) ;{ }^{13} \mathrm{C} \mathrm{NMR}(100 \mathrm{MHz}$, $\left.\mathrm{CDCl}_{3}\right) \delta 176.6\left(\mathrm{~d}, J_{\mathrm{CF}}=272.0 \mathrm{~Hz}, 1 \mathrm{C}\right), 164.5\left(\mathrm{~d}, J_{\mathrm{CF}}=21.7 \mathrm{~Hz}, 1 \mathrm{C}\right), 98.1\left(\mathrm{~d}, J_{\mathrm{CF}}=27.9 \mathrm{~Hz}, 1 \mathrm{C}\right)$, 66.6, 66.5, 46.4, 41.7, $38.3\left(\mathrm{~d}, J_{\mathrm{CF}}=22.0 \mathrm{~Hz}, 1 \mathrm{C}\right), 31.5,28.5(2 \mathrm{C}), 25.5(2 \mathrm{C}) ;{ }^{19} \mathrm{~F} \mathrm{NMR}(471 \mathrm{MHz}$, $\left.\mathrm{CDCl}_{3}\right) \delta-93.4\left(\mathrm{dd}, J_{\mathrm{HF}}=33.0,21.2 \mathrm{~Hz}, 1 \mathrm{~F}\right)$; IR (film) $v_{\max } 2928,2853,1631,1433,1114 \mathrm{~cm}^{-1}$; HRMS (ESI-TOF) $m / z$ calcd for $\mathrm{C}_{13} \mathrm{H}_{20} \mathrm{FNO}_{2} \mathrm{Cs}[\mathrm{M}+\mathrm{Cs}]^{+} 374.0533$, found 374.0541.

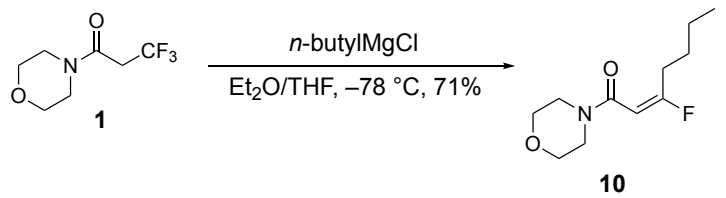

(E)-3-fluoro-1-morpholinohept-2-en-1-one (10). A solution of $\mathbf{1}(100 \mathrm{mg}, 0.51 \mathrm{mmol})$ in $\mathrm{THF} / \mathrm{Et}_{2} \mathrm{O}(1: 1,3 \mathrm{~mL})$ was cooled to $-78^{\circ} \mathrm{C}$, treated with a solution of $n$-butylmagnesium chloride $\left(1.0 \mathrm{~mL}, 2.0 \mathrm{M}\right.$ in $\mathrm{Et}_{2} \mathrm{O}$ ), and stirred for $4 \mathrm{~h}$. The reaction mixture was quenched with $0.2 \mathrm{~N} \mathrm{HCl}$ solution $(3 \mathrm{~mL})$ and extracted with $\mathrm{CH}_{2} \mathrm{Cl}_{2}(3 \times 5 \mathrm{~mL})$. The combined organics were washed with saturated aqueous $\mathrm{NaCl}(10 \mathrm{~mL})$, dried over $\mathrm{Na}_{2} \mathrm{SO}_{4}$, and concentrated under reduced pressure. $\mathrm{SiO}_{2}$ flash chromatography $(20 \rightarrow 80 \%$ EtOAc in hexanes $)$ afforded the title compound $\mathbf{1 0}$ as a colorless oil $(78 \mathrm{mg}, 71 \%):{ }^{1} \mathrm{H}$ NMR $\left(500 \mathrm{MHz} \mathrm{CDCl}_{3}\right) \delta 5.78(\mathrm{~d}, \mathrm{~J}=20.8 \mathrm{~Hz}, 1 \mathrm{H}), 3.68-3.45$ $(\mathrm{m}, 8 \mathrm{H}), 2.68(\mathrm{dt}, J=25.2,7.6 \mathrm{~Hz}, 2 \mathrm{H}), 1.57(\mathrm{p}, J=7.6 \mathrm{~Hz}, 2 \mathrm{H}), 1.38(\mathrm{q}, J=7.5 \mathrm{~Hz}, 2 \mathrm{H}), 0.91(\mathrm{t}$, $J=7.4 \mathrm{~Hz}, 3 \mathrm{H}) ;{ }^{13} \mathrm{C} \mathrm{NMR}\left(100 \mathrm{MHz}, \mathrm{CDCl}_{3}\right) \delta 173.9\left(\mathrm{~d}, J_{\mathrm{CF}}=269.0 \mathrm{~Hz}, 1 \mathrm{C}\right), 164.6\left(\mathrm{~d}, J_{\mathrm{CF}}=\right.$ $21.5 \mathrm{~Hz}, 1 \mathrm{C}), 99.7\left(\mathrm{~d}, J_{\mathrm{CF}}=27.1 \mathrm{~Hz}, 1 \mathrm{C}\right), 66.8,66.6,46.5,41.8,29.5$ (d, $\left.J_{\mathrm{CF}}=23.4 \mathrm{~Hz}, 1 \mathrm{C}\right), 28.0$, 
22.2, 13.7; ${ }^{19} \mathrm{~F} \mathrm{NMR}\left(376 \mathrm{MHz}, \mathrm{CDCl}_{3}\right) \delta-81.7\left(\mathrm{td}, J_{\mathrm{HF}}=25.2,21.0 \mathrm{~Hz}, 1 \mathrm{~F}\right) ; \mathrm{IR}\left(\right.$ film) $v_{\max } 2859$, $1675,1619,1426,1113 \mathrm{~cm}^{-1}$; HRMS (ESI-TOF) $\mathrm{m} / z$ calcd for $\mathrm{C}_{11} \mathrm{H}_{18} \mathrm{FNO}_{2} \mathrm{Cs}[\mathrm{M}+\mathrm{Cs}]^{+} 348.0376$, found 348.0348 .

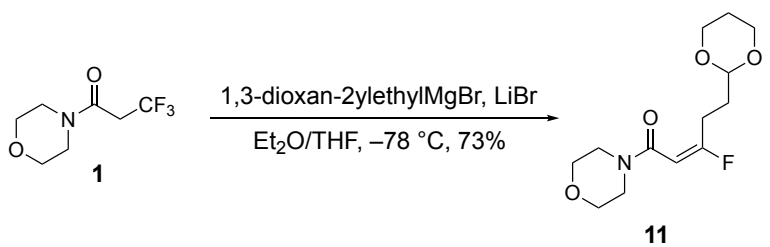

(E)-5-(1,3-dioxan-2-yl)-3-fluoro-1-morpholinopent-2-en-1-one (11). A solution of 1 (212 mg, $1.07 \mathrm{mmol})$ and $\mathrm{LiBr}(186 \mathrm{mg}, 2.2 \mathrm{mmol})$ in THF/Et $2 \mathrm{O}(1: 1,2 \mathrm{~mL})$ was cooled to $-78^{\circ} \mathrm{C}$, treated with a solution of 1,3-dioxan-2-ylethylmagnesium bromide (4.0 mL, $0.5 \mathrm{M}$ in THF), and stirred for $4 \mathrm{~h}$. The reaction mixture was quenched with $0.2 \mathrm{~N} \mathrm{HCl}$ solution $(2 \mathrm{~mL})$ and extracted with $\mathrm{CH}_{2} \mathrm{Cl}_{2}(3 \times 5 \mathrm{~mL})$. The combined organics were washed with saturated aqueous $\mathrm{NaCl}(10 \mathrm{~mL})$, dried over $\mathrm{Na}_{2} \mathrm{SO}_{4}$, and concentrated under reduced pressure. $\mathrm{SiO}_{2}$ flash chromatography $(20 \rightarrow 80 \%$ EtOAc in hexanes) afforded the title compound $\mathbf{1 1}$ as a solid $(213 \mathrm{mg}, 73 \%)$. Recrystallization from toluene (by slow evaporation) provided a crystalline solid suitable for Xray structure analysis: $\mathrm{mp} 55-56{ }^{\circ} \mathrm{C} ;{ }^{1} \mathrm{H} \mathrm{NMR}\left(500 \mathrm{MHz}, \mathrm{CDCl}_{3}\right) \delta 5.79(\mathrm{~d}, J=20.4 \mathrm{~Hz}, 1 \mathrm{H}), 4.58$ $(\mathrm{t}, J=5.1 \mathrm{~Hz}, 1 \mathrm{H}), 4.09$ (dd, $J=10.7,5.0 \mathrm{~Hz}, 2 \mathrm{H}), 3.74(\mathrm{td}, J=12.4,2.5 \mathrm{~Hz}, 2 \mathrm{H}), 3.69-3.60(\mathrm{~m}$, $6 \mathrm{H}), 3.50-3.44(\mathrm{~m}, 2 \mathrm{H}), 2.81(\mathrm{t}, J=8.0 \mathrm{~Hz}, 1 \mathrm{H}), 2.76(\mathrm{t}, J=8.0 \mathrm{~Hz}, 1 \mathrm{H}), 2.06(\mathrm{dtt}, J=13.4,12.5$, $5.0 \mathrm{~Hz}, 1 \mathrm{H}), 1.90-1.84(\mathrm{~m}, 2 \mathrm{H}), 1.32(\mathrm{dt}, J=13.5,1.4 \mathrm{~Hz}, 1 \mathrm{H}) ;{ }^{13} \mathrm{C}$ NMR $\left(100 \mathrm{MHz}, \mathrm{CDCl}_{3}\right) \delta$ $172.9\left(\mathrm{~d}, J_{\mathrm{CF}}=269.4 \mathrm{~Hz}, 1 \mathrm{C}\right), 164.4\left(\mathrm{~d}, J_{\mathrm{CF}}=20.7 \mathrm{~Hz}, 1 \mathrm{C}\right), 101.1,100.1\left(\mathrm{~d}, J_{\mathrm{CF}}=26.5 \mathrm{~Hz}, 1 \mathrm{C}\right)$, $66.8(2 \mathrm{C}), 66.6(2 \mathrm{C}), 46.5,41.9,31.3,25.7,24.7$ (d, $\left.J_{\mathrm{CF}}=23.9 \mathrm{~Hz}, 1 \mathrm{C}\right) ;{ }^{19} \mathrm{~F}$ NMR $(376 \mathrm{MHz}$, $\left.\mathrm{CDCl}_{3}\right) \delta-82.6\left(\mathrm{td}, J_{\mathrm{HF}}=24.3,20.9 \mathrm{~Hz}, 1 \mathrm{~F}\right)$; IR (film) $v_{\max } 2851,1677,1619,1429,1113 \mathrm{~cm}^{-1}$; HRMS (ESI-TOF) $m / z$ calcd for $\mathrm{C}_{13} \mathrm{H}_{20} \mathrm{FNO}_{4} \mathrm{Cs}[\mathrm{M}+\mathrm{Cs}]^{+} 406.0431$, found 406.0439.

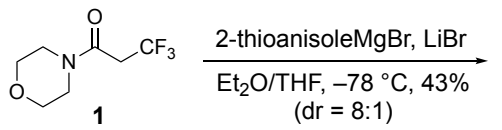

(E)-3-fluoro-3-(2-(methylthio)phenyl)-1-morpholinoprop-2-en-1-one (12). A solution of 2thioanisolemagnesium bromide $(2.0 \mathrm{~mL}, 0.5 \mathrm{M}$ in THF) and $\mathrm{LiBr}(88.6 \mathrm{mg}, 1.0 \mathrm{mmol})$ in $1: 1$ $\mathrm{THF} / \mathrm{Et}_{2} \mathrm{O}(10 \mathrm{~mL})$ was stirred at $\mathrm{rt}$ for $1 \mathrm{~h}$, cooled to $-78^{\circ} \mathrm{C}$, treated with a solution of $\mathbf{1}(100 \mathrm{mg}$, $0.5 \mathrm{mmol})$ in THF $(7 \mathrm{~mL})$, and then stirred for $8 \mathrm{~h}$. The reaction mixture was quenched with $0.2 \mathrm{~N}$ $\mathrm{HCl}$ solution $(2 \mathrm{~mL})$ and extracted with $\mathrm{CH}_{2} \mathrm{Cl}_{2}(3 \times 5 \mathrm{~mL})$. The combined organics were washed with saturated aqueous $\mathrm{NaCl}(10 \mathrm{~mL})$, dried over $\mathrm{Na}_{2} \mathrm{SO}_{4}$, and concentrated under reduced pressure. $\mathrm{SiO}_{2}$ flash chromatography $(5 \rightarrow 80 \%$ EtOAc in hexanes) then preparative TLC (25\% $\mathrm{Et}_{2} \mathrm{O}$ in $\left.\mathrm{CH}_{2} \mathrm{Cl}_{2}\right)$ afforded the title compound 12 as a pale yellow oil $(62 \mathrm{mg}, 43 \%)$ as a 8:1 mixture of E/Z-isomers: ${ }^{1} \mathrm{H}$ NMR $\left(400 \mathrm{MHz}, \mathrm{CDCl}_{3}\right) \delta 7.43-7.37(\mathrm{~m}, 2 \mathrm{H}), 7.31(\mathrm{~d}, J=8.0 \mathrm{~Hz}, 1 \mathrm{H}), 7.19$ $(\mathrm{t}, J=7.5 \mathrm{~Hz}, 1 \mathrm{H}), 6.05(\mathrm{~d}, J=16.5,1 \mathrm{H}), 5.84(\mathrm{~d}, J=37.5 \mathrm{~Hz}, 1 \mathrm{H})^{*}, 3.48(\mathrm{~m}, 4 \mathrm{H}), 3.41(\mathrm{~m}, 2 \mathrm{H})$, $3.26(\mathrm{~m}, 2 \mathrm{H}), 2.49(\mathrm{~s}, 3 \mathrm{H}) ;{ }^{13} \mathrm{C} \mathrm{NMR}\left(100 \mathrm{MHz}, \mathrm{CDCl}_{3}\right) \delta 163.7\left(\mathrm{~d}, J_{\mathrm{CF}}=17.4 \mathrm{~Hz}, 1 \mathrm{C}\right), 163.3(\mathrm{~d}$, $\left.J_{\mathrm{CF}}=263.3 \mathrm{~Hz}, 1 \mathrm{C}\right), 138.3,131.1\left(\mathrm{~d}, J_{\mathrm{CF}}=2.2 \mathrm{~Hz}, 1 \mathrm{C}\right), 130.6\left(\mathrm{~d}, J_{\mathrm{CF}}=3.4 \mathrm{~Hz}, 1 \mathrm{C}\right), 129.9\left(\mathrm{~d}, J_{\mathrm{CF}}\right.$ $=24.8 \mathrm{~Hz}, 1 \mathrm{C}), 126.2,124.9,104.5\left(\mathrm{~d}, J_{\mathrm{CF}}=26.8 \mathrm{~Hz}, 1 \mathrm{C}\right), 66.5(2 \mathrm{C}), 46.9,41.9,16.2 ;{ }^{19} \mathrm{~F} \mathrm{NMR}$ $\left(376 \mathrm{MHz}, \mathrm{CDCl}_{3}\right) \delta-83.1(\mathrm{~d}, J=16.4 \mathrm{~Hz}),-87.8(\mathrm{~d}, J=37.7 \mathrm{~Hz})^{*}$; IR (film) $v_{\max } 2920,2855$, $1675,1627,1435,1112 \mathrm{~cm}^{-1}$; HRMS (ESI-TOF) $\mathrm{m} / z$ calcd for $\mathrm{C}_{14} \mathrm{H}_{17} \mathrm{FNO}_{2} \mathrm{~S}[\mathrm{M}+\mathrm{H}]^{+} 282.0964$, found 282.0944 . *denotes the minor $(Z)$-isomer. 

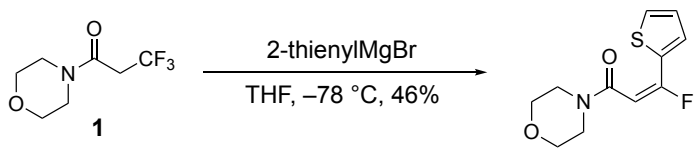

13

(E)-3-fluoro-1-morpholino-3-(thiophen-2-yl)prop-2-en-1-one (13). A solution of 1 (100 mg, 0.51 $\mathrm{mmol})$ in THF $(17 \mathrm{~mL})$ was cooled to $-78{ }^{\circ} \mathrm{C}$, treated with a solution of 2-thienylmagnesium bromide (1.0 mL, 1.0 M in THF), and stirred for $4 \mathrm{~h}$. The reaction mixture was quenched with 0.2 $\mathrm{N} \mathrm{HCl}$ solution $(3 \mathrm{~mL})$ and extracted with $\mathrm{CH}_{2} \mathrm{Cl}_{2}(3 \times 5 \mathrm{~mL})$. The combined organics were washed with saturated aqueous $\mathrm{NaCl}(10 \mathrm{~mL})$, dried over $\mathrm{Na}_{2} \mathrm{SO}_{4}$, and concentrated under reduced pressure. $\mathrm{SiO}_{2}$ flash chromatography $(5 \rightarrow 60 \%$ EtOAc in hexanes) afforded the title compound 13 as a colorless oil $(57 \mathrm{mg}, 46 \%):{ }^{1} \mathrm{H} \mathrm{NMR}\left(500 \mathrm{MHz}, \mathrm{CDCl}_{3}\right) \delta 7.57(\mathrm{dd}, J=3.8,1.2 \mathrm{~Hz}, 1 \mathrm{H}), 7.47$ (ddd, $J=5.6,2.8,1.4 \mathrm{~Hz}, 1 \mathrm{H}), 7.05(\mathrm{ddd}, J=5.1,3.9,1.6 \mathrm{~Hz}, 1 \mathrm{H}), 5.84(\mathrm{~d}, J=21.4 \mathrm{~Hz}, 1 \mathrm{H}), 3.67$ $(\mathrm{m}, 4 \mathrm{H}), 3.55(\mathrm{~m}, 2 \mathrm{H}), 3.48(\mathrm{~m}, 2 \mathrm{H}) ;{ }^{13} \mathrm{C} \mathrm{NMR}\left(100 \mathrm{MHz}, \mathrm{CDCl}_{3}\right) \delta 163.8\left(\mathrm{~d}, J_{\mathrm{CF}}=19.1 \mathrm{~Hz}\right)$, $158.7\left(\mathrm{~d}, J_{\mathrm{CF}}=249.4 \mathrm{~Hz}\right), 131.5\left(\mathrm{~d}, J_{\mathrm{CF}}=33.3 \mathrm{~Hz}\right), 130.0,129.9\left(\mathrm{~d}, J_{\mathrm{CF}}=6.9 \mathrm{~Hz}\right), 127.0,99.1(\mathrm{~d}$, $\left.J_{\mathrm{CF}}=29.8 \mathrm{~Hz}\right), 66.5(2 \mathrm{C}), 46.7,41.8 ;{ }^{19} \mathrm{~F} \mathrm{NMR}\left(471 \mathrm{MHz}, \mathrm{CDCl}_{3}\right) \delta-92.1(\mathrm{~d}, J=21.0 \mathrm{~Hz})$; IR (film) $v_{\max } 2853,2201,1620,1415,1111 \mathrm{~cm}^{-1}$; HRMS (ESI-TOF) $\mathrm{m} / z$ calcd for $\mathrm{C}_{11} \mathrm{H}_{13} \mathrm{FNO}_{2} \mathrm{~S}$ $[\mathrm{M}+\mathrm{H}]^{+} 242.0651$, found 242.0670 .

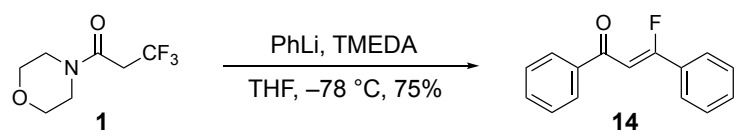

(Z)-3-fluoro-1,3-diphenylprop-2-en-1-one (14). A solution of 1 (100 $\mathrm{mg}, 0.51 \mathrm{mmol})$ and TMEDA $(0.15 \mathrm{ml}, 1.02 \mathrm{mmol})$ in THF $(2 \mathrm{~mL})$ was cooled to $-78^{\circ} \mathrm{C}$, treated with a solution of phenyllithium $(1.0 \mathrm{~mL}, 1.9 \mathrm{M}$ in dibutylether), and stirred for $4 \mathrm{~h}$. The reaction mixture was quenched with $0.2 \mathrm{~N} \mathrm{HCl}$ solution $(2 \mathrm{~mL})$ and extracted with $\mathrm{CH}_{2} \mathrm{Cl}_{2}(3 \times 5 \mathrm{~mL})$. The combined organics were washed with saturated aqueous $\mathrm{NaCl}(10 \mathrm{~mL})$, dried over $\mathrm{Na}_{2} \mathrm{SO}_{4}$, and concentrated under reduced pressure. $\mathrm{SiO}_{2}$ flash chromatography $(5 \rightarrow 80 \%$ EtOAc in hexanes $)$ afforded the title compound 14 as yellow oil (105 mg, 75\%): ${ }^{1} \mathrm{H}$ NMR $\left(400 \mathrm{MHz}, \mathrm{CDCl}_{3}\right) \delta 7.97(\mathrm{~d}, J=7.4 \mathrm{~Hz}$, 2H), $7.75(\mathrm{~d}, J=6.8 \mathrm{~Hz}, 2 \mathrm{H}), 7.75(\mathrm{~d}, J=6.8 \mathrm{~Hz}, 1 \mathrm{H}), 7.52-7.44(\mathrm{~m}, 5 \mathrm{H}), 6.80(\mathrm{~d}, J=34.2 \mathrm{~Hz}$, $1 \mathrm{H}) ;{ }^{13} \mathrm{C} \mathrm{NMR}\left(100 \mathrm{MHz}, \mathrm{CDCl}_{3}\right) \delta 188.9,165.2\left(\mathrm{~d}, J_{\mathrm{CF}}=276.5 \mathrm{~Hz}, 1 \mathrm{C}\right), 138.6,132.9,131.6$, $130.9\left(\mathrm{~d}, J_{\mathrm{CF}}=26.2 \mathrm{~Hz}, 1 \mathrm{C}\right), 128.9\left(\mathrm{~d}, J_{\mathrm{CF}}=2.1 \mathrm{~Hz}, 2 \mathrm{C}\right), 128.6(2 \mathrm{C}), 128.3(2 \mathrm{C}), 125.8\left(\mathrm{~d}, J_{\mathrm{CF}}=\right.$ $8.0 \mathrm{~Hz}, 2 \mathrm{C}), 101.7\left(\mathrm{~d}, J_{\mathrm{CF}}=6.9 \mathrm{~Hz}, 1 \mathrm{C}\right) ;{ }^{19} \mathrm{~F} \mathrm{NMR}\left(376 \mathrm{MHz}, \mathrm{CDCl}_{3}\right) \delta-97.6\left(\mathrm{~d}, J_{\mathrm{HF}}=34.2 \mathrm{~Hz}\right.$, 1F); IR (film) $v_{\max } 3058,1636,1284,1208 \mathrm{~cm}^{-1}$; HRMS (ESI-TOF) $\mathrm{m} / z$ calcd for $\mathrm{C}_{15} \mathrm{H}_{11} \mathrm{FOCs}$ $[\mathrm{M}+\mathrm{Cs}]^{+}$358.9848, found 358.9846. All data matched the reported data. ${ }^{1}$

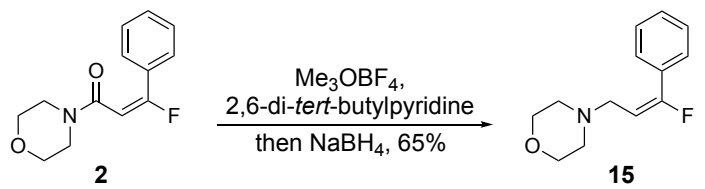

(E)-4-(3-fluoro-3-phenylallyl)morpholine (15). A solution of amide 2 (341 $\mathrm{mg}, 1.45 \mathrm{mmol})$ in $\mathrm{CH}_{2} \mathrm{Cl}_{2}(24 \mathrm{~mL})$ was treated with trimethyloxonium tetrafluoroborate $(578 \mathrm{mg}, 3.91 \mathrm{mmol})$ and 2,6-di-tert-butylpyridine $(0.94 \mathrm{~mL}, 4.22 \mathrm{mmol})$, and the reaction mixture was stirred for $24 \mathrm{~h}$ at $\mathrm{rt}$. Next, the reaction mixture was cooled to $0{ }^{\circ} \mathrm{C}$, diluted with methanol $(15 \mathrm{~mL})$, and stirred for 20 min at $0{ }^{\circ} \mathrm{C} . \mathrm{NaBH}_{4}(564 \mathrm{mg}, 14.9 \mathrm{mmol})$ was added and the resultant mixture was stirred for 30 min at $0{ }^{\circ} \mathrm{C}$. The reaction mixture was quenched with saturated aqueous $\mathrm{NaHCO}_{3}(20 \mathrm{~mL})$ and diluted with $\mathrm{CH}_{2} \mathrm{Cl}_{2}(15 \mathrm{~mL})$. The aqueous layer was extracted with $\mathrm{CH}_{2} \mathrm{Cl}_{2}(3 \times 20 \mathrm{~mL})$. The 
combined organics were dried over $\mathrm{Na}_{2} \mathrm{SO}_{4}$ and concentrated under reduced pressure. $\mathrm{SiO}_{2}$ flash chromatography ( $7: 3$ hexanes/EtOAc with $0.5 \% \mathrm{AcOH} \rightarrow 7: 3$ hexanes/EtOAc with $\left.0.5 \% \mathrm{Et}_{3} \mathrm{~N}\right)$ afforded the title compound 15 as colorless oil $(27 \mathrm{mg}, 65 \%):{ }^{1} \mathrm{H}$ NMR $\left(400 \mathrm{MHz}, \mathrm{CDCl}_{3}\right) \delta 7.49$ $7.45(\mathrm{~m}, 2 \mathrm{H}), 7.43-7.38(\mathrm{~m}, 3 \mathrm{H}), 5.53(\mathrm{dt}, J=21.4,7.5 \mathrm{~Hz}, 1 \mathrm{H}), 3.72(\mathrm{t}, J=4.7 \mathrm{~Hz}, 4 \mathrm{H}), 3.15(\mathrm{~d}$, $J=7.5 \mathrm{~Hz}, 2 \mathrm{H}), 2.47(\mathrm{~m}, 4 \mathrm{H}) ;{ }^{13} \mathrm{C} \mathrm{NMR}\left(100 \mathrm{MHz}, \mathrm{CDCl}_{3}\right) \delta 159.3\left(\mathrm{~d}, J_{\mathrm{CF}}=246.0 \mathrm{~Hz}, 1 \mathrm{C}\right), 131.4$ $\left(\mathrm{d}, J_{\mathrm{CF}}=29.4 \mathrm{~Hz}, 1 \mathrm{C}\right), 129.4\left(\mathrm{~d}, J_{\mathrm{CF}}=1.4 \mathrm{~Hz}, 1 \mathrm{C}\right), 128.3(2 \mathrm{C}), 128.0\left(\mathrm{~d}, J_{\mathrm{CF}}=4.8 \mathrm{~Hz}, 2 \mathrm{C}\right), 104.5$ $\left(\mathrm{d}, J_{\mathrm{CF}}=26.7 \mathrm{~Hz}, 1 \mathrm{C}\right), 66.8(2 \mathrm{C}), 54.4\left(\mathrm{~d}, J_{\mathrm{CF}}=10.1 \mathrm{~Hz}, 1 \mathrm{C}\right), 53.3(2 \mathrm{C}) ;{ }^{19} \mathrm{~F}$ NMR $(376 \mathrm{MHz}$, $\left.\mathrm{CDCl}_{3}\right) \delta-95.0\left(\mathrm{~d}, J_{\mathrm{HF}}=21.1 \mathrm{~Hz}, 1 \mathrm{~F}\right)$; IR (film) $v_{\max } 2959,2918,2853,2808,1677,1115,1060$ $\mathrm{cm}^{-1}$; HRMS (ESI-TOF) $\mathrm{m} / z$ calcd for $\mathrm{C}_{13} \mathrm{H}_{17} \mathrm{FNO}[\mathrm{M}+\mathrm{H}]^{+} 222.1294$ found 222.1296 .

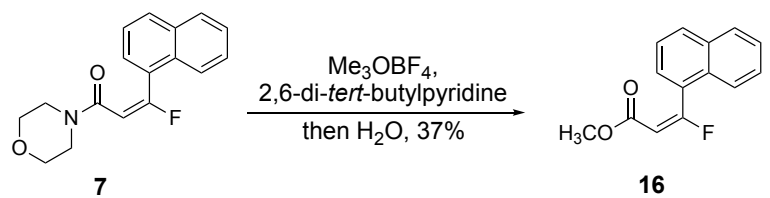

Methyl (E)-3-fluoro-3-(naphthalen-1-yl)acrylate (16). A solution of amide 7 (38 $\mathrm{mg}, 0.13$ $\mathrm{mmol})$ in $\mathrm{CH}_{2} \mathrm{Cl}_{2}(5 \mathrm{~mL})$ was treated with trimethyloxonium tetrafluoroborate (97 $\left.\mathrm{mg}, 0.66 \mathrm{mmol}\right)$ and stirred for $24 \mathrm{~h}$ at $\mathrm{rt}$. The resultant mixture was concentrated under reduced pressure, diluted with THF $(3 \mathrm{~mL})$, treated with $\mathrm{H}_{2} \mathrm{O}(2 \mathrm{~mL})$, and stirred for $1 \mathrm{~h}$ at $\mathrm{rt}$. Next, the mixture was added to $\mathrm{H}_{2} \mathrm{O}(4 \mathrm{~mL})$ and acidified to $\mathrm{pH}$. Then, the mixture was extracted with $\mathrm{Et}_{2} \mathrm{O}(3 \times 10 \mathrm{~mL})$. The combined organics were dried over $\mathrm{Na}_{2} \mathrm{SO}_{4}$ and concentrated under reduced pressure. $\mathrm{SiO}_{2}$ flash chromatography (8:2 hexanes/EtOAc) afforded the title compound $\mathbf{1 6}$ as a colorless oil (11.1 mg, 37\%): ${ }^{1} \mathrm{H}$ NMR (500 MHz, $\left.\mathrm{CDCl}_{3}\right) \delta$ 7.99-7.89 (m, 3H), $7.64(\mathrm{~m}, 1 \mathrm{H}), 7.57-7.49(\mathrm{~m}, 3 \mathrm{H}), 6.18$ $(\mathrm{d}, J=16.8 \mathrm{~Hz}, 1 \mathrm{H}), 3.55(\mathrm{~s}, 3 \mathrm{H}) ;{ }^{13} \mathrm{C} \mathrm{NMR}\left(125 \mathrm{MHz}, \mathrm{CDCl}_{3}\right) \delta 170.8\left(\mathrm{~d}, J_{\mathrm{CF}}=271.0 \mathrm{~Hz}, 1 \mathrm{C}\right)$, $165.2\left(\mathrm{~d}, J_{\mathrm{CF}}=23.5 \mathrm{~Hz}, 1 \mathrm{C}\right), 133.2\left(\mathrm{~d}, J_{\mathrm{CF}}=1.7 \mathrm{~Hz}, 1 \mathrm{C}\right), 131.2\left(\mathrm{~d}, J_{\mathrm{CF}}=3.0 \mathrm{~Hz}, 1 \mathrm{C}\right), 130.6,128.8$, $128.8,128.5,128.0\left(\mathrm{~d}, J_{\mathrm{CF}}=24.0 \mathrm{~Hz}, 1 \mathrm{C}\right), 127.1\left(\mathrm{~d}, J_{\mathrm{CF}}=1.2 \mathrm{~Hz}, 1 \mathrm{C}\right), 126.3,124.6,104.3\left(\mathrm{~d}, J_{\mathrm{CF}}\right.$ $=32.0 \mathrm{~Hz}, 1 \mathrm{C}), 51.5 ;{ }^{19} \mathrm{~F} \mathrm{NMR}\left(376 \mathrm{MHz}, \mathrm{CDCl}_{3}\right) \delta-66.2\left(\mathrm{~d}, J_{\mathrm{HF}}=16.8 \mathrm{~Hz}, 1 \mathrm{~F}\right)$; IR (film) $v_{\max }$ 2952, 1731, 1666, 1202, $1101 \mathrm{~cm}^{-1}$; HRMS (ESI-TOF) $\mathrm{m} / z$ calcd for $\mathrm{C}_{14} \mathrm{H}_{10} \mathrm{FO}_{2}[\mathrm{M}-\mathrm{H}]^{-}$ 229.0665 , found 229.0663 .

\section{Mechanistic Experiments}

Observation of diagnostic peaks the $\beta, \beta$-difluoroacrylamide by ${ }^{19} \mathbf{F}$ NMR. A solution of isopropylmagnesium chloride in THF- $d_{8}$ was cooled to $-78{ }^{\circ} \mathrm{C}$, treated with a solution of $\mathbf{1}$ (one equiv.) in THF- $d_{8}$, and then stirred for $18 \mathrm{~h}$ at $\mathrm{rt}$. The mixture was analyzed by ${ }^{19} \mathrm{~F}$ NMR (471 $\mathrm{MHz}, \mathrm{THF}-d_{8}$ ) at rt. The diagnostic peaks are observed at -71.2 and $-76.0 \mathrm{ppm}$ (Figure S1, see below) and they are similar to the $\beta, \beta$-difluoroacrylamide intermediate reported by Shimada, Konno, and Ishihara. ${ }^{2}$ 


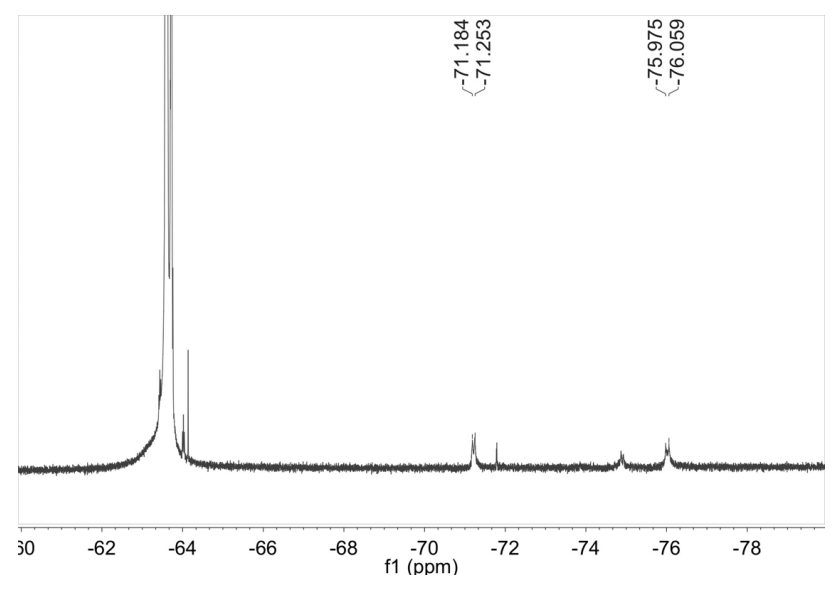

Figure S1. ${ }^{19} \mathrm{~F}$ NMR data for the $\beta, \beta$-difluoroacrylamide intermediate was obtained from $\mathbf{1}$ and isopropylmagnesium chloride in THF- $d_{8}$ at $471 \mathrm{~Hz}$ at $\mathrm{rt}$ with trifluorotoluene as an internal standard.

\section{References}

1. Zhang, J.; Liu, L.; Duan, J.; Gu, L.; Chen, B.; Sun, T.; Gong, Y. Stereoselective One-Pot Sequential Dehydrochlorination/trans-Hydrofluorination Reaction of $\beta$-Chloro- $\alpha, \beta$ unsaturated Aldehydes or Ketones: Facile Access to (Z)- $\beta$-Fluoro- $\beta$-arylenals/ $\beta$-Fluoro- $\beta$ arylenones. Adv. Synth. Catal. 2017, 359, 4348-4358.

2. Shimada, T.; Konno, T.; Ishihara, T. A New Access to 3-Halo-3,3-difluoropropanoic Acid Derivatives via Fluorine-Halogen Exchange Reaction of Silyl Enolates of 3,3,3Trifluoropropanoic Acid Derivatives. Chem. Lett. 2007, 36, 636-637.

\section{X-ray Experimental and Crystallographic Data}

The X-ray crystal structures of $\mathbf{1}$ and $\mathbf{1 1}$ were determined at T=90K from data collected with Mo $\mathrm{K} \alpha$ radiation on a Bruker Kappa Apex-II DUO diffractometer equipped with a Triumph curved monochromator. Refinement was by SHELXL using all data, with $\mathrm{H}$ atoms in idealized positions. 1: Recrystallization from a 1:1 solution of hexanes and cyclohexanes (by slow evaporation) provided a crystalline solid suitable for $\mathrm{X}$-ray structure analysis. $\mathrm{C}_{7} \mathrm{H}_{10} \mathrm{~F}_{3} \mathrm{NO}_{2}, \mathrm{FW}=197.16$, monoclinic space group $\mathrm{P} 21 / \mathrm{n}, \mathrm{a}=12.5889(4), \mathrm{b}=10.7958(3), \mathrm{c}=12.6859(4) \AA, \beta=96.670(2)^{\circ}$, $\mathrm{V}=1712.44(9) \AA^{3}, \mathrm{Z}=8.14,586$ data measured to $\theta_{\max }=33.2^{\circ}$ yielded 6524 unique data $\left(\mathrm{R}_{\text {int }}=0.039\right)$, of which 3902 had $\mathrm{I}>2 \sigma(\mathrm{I}), \mathrm{R}=0.048$. There are two independent (unrelated by symmetry) molecules, one of which exhibits disorder of the morpholine ring, the two conformations being present in 0.927/0.073 ratio. The CIF has been deposited at the Cambridge Crystallographic Data Centre, CCDC 1948771.

11: Recrystallization from toluene (by slow evaporation) provided a crystalline solid suitable for X-ray structure analysis. $\mathrm{C}_{13} \mathrm{H}_{20} \mathrm{FNO}_{4}, \mathrm{FW}=273.30$, orthorhombic space group $\mathrm{P} 2{ }_{1} 2_{1} 2_{1}$, $\mathrm{a}=4.2875(5), \mathrm{b}=10.9693(12), \mathrm{c}=27.265(4) \AA, \mathrm{V}=1282.3(3) \AA^{3}, \mathrm{Z}=4.4579$ data measured to $\theta_{\max }=26.4^{\circ}$ yielded 2501 unique data $\left(R_{\text {int }}=0.048\right)$, of which 1750 had $I>2 \sigma(I), R=0.066$. The 
morpholine ring has disorder similar to that seen in $\mathbf{1}$, the two conformations being present in 0.888/0.112 ratio. CCDC 1948772.

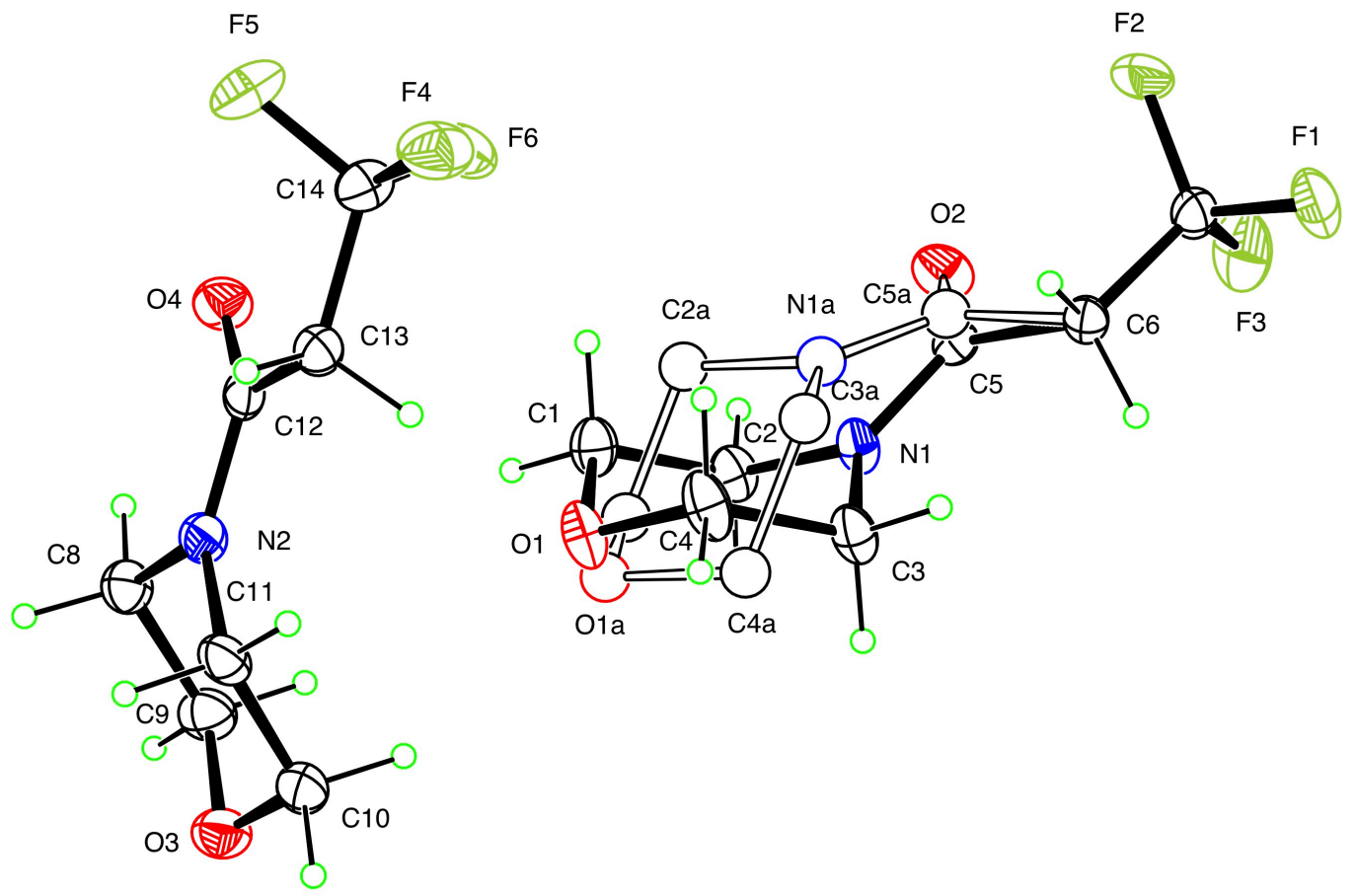

Figure S2. ORTEP diagram of 1 with 50\% ellipsoids, showing disorder of the morpholine ring.

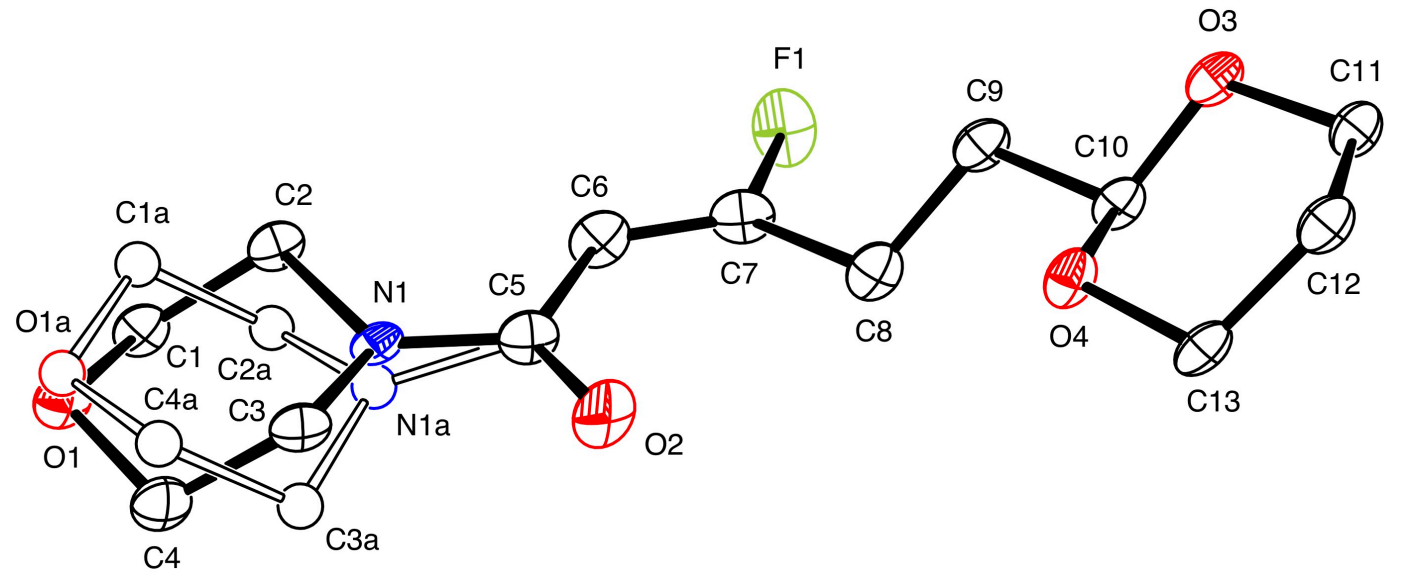

Figure S3. ORTEP diagram of 11 with 50\% ellipsoids, showing disorder of the morpholine ring. $\mathrm{H}$ atoms are not shown. 


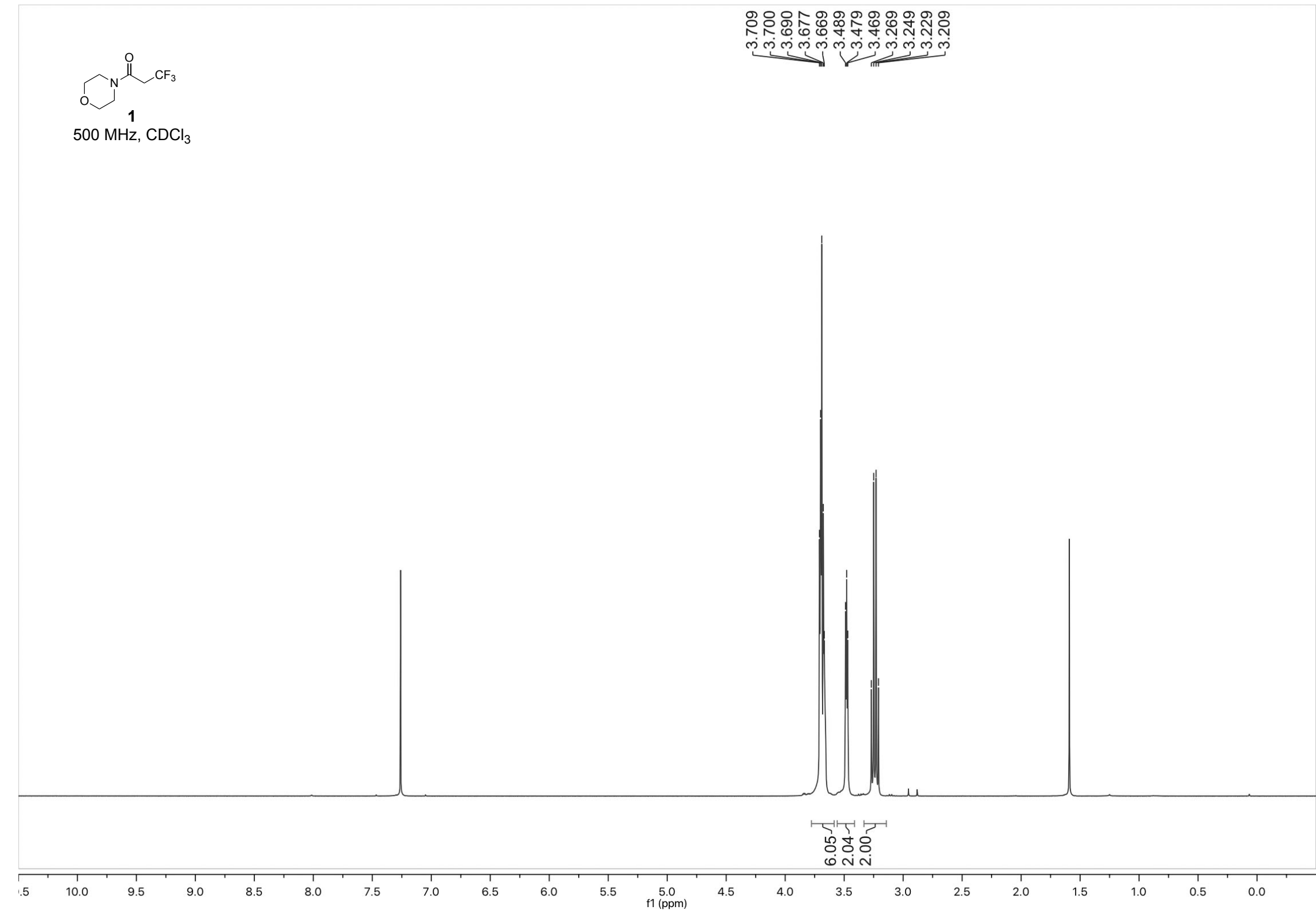




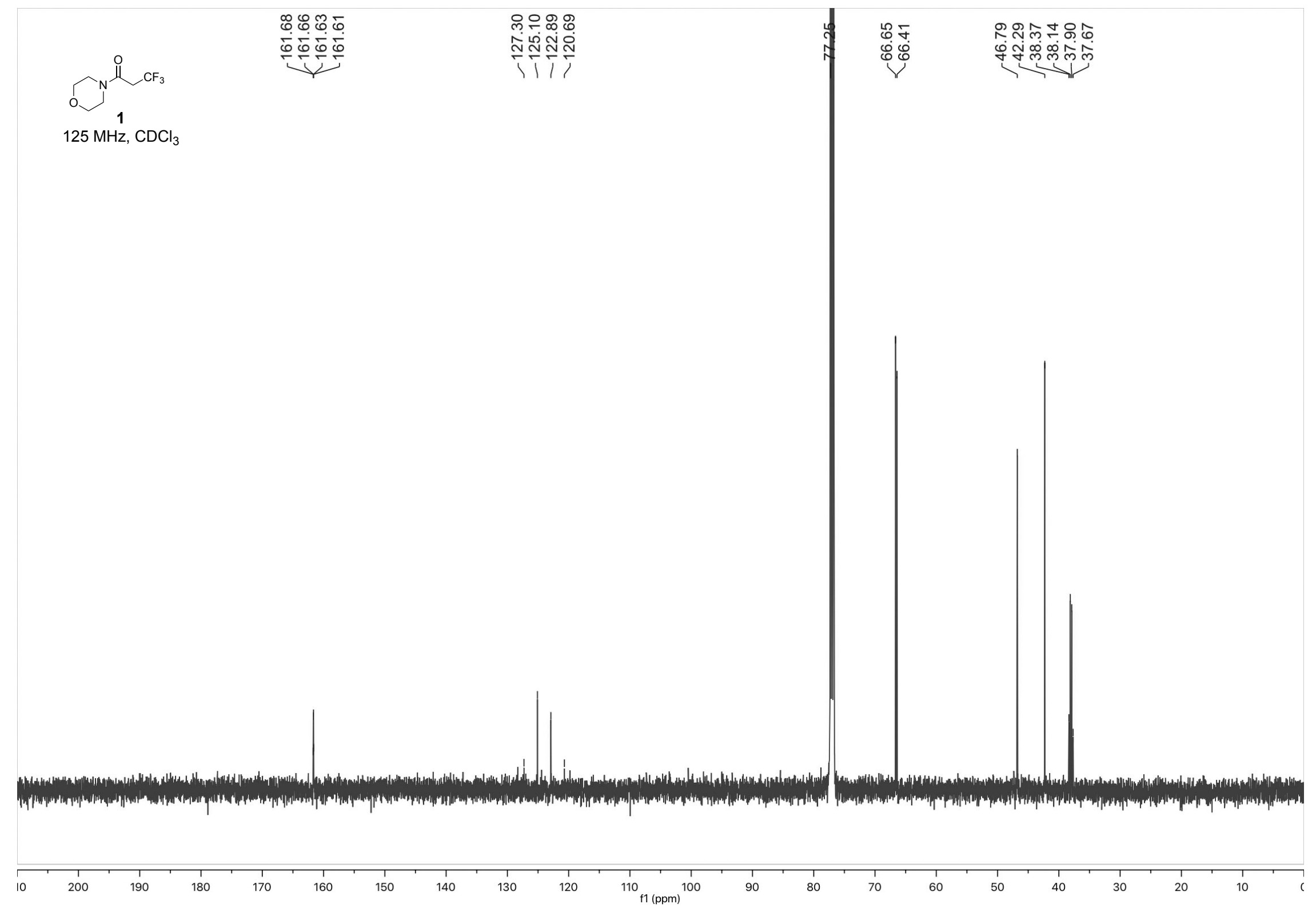


$\overbrace{}^{N} \stackrel{\text { 少 }}{C^{2} F_{3}}$

$376 \mathrm{MHz}, \mathrm{CDCl}_{3}$

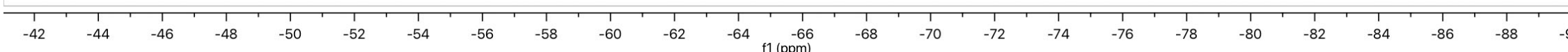




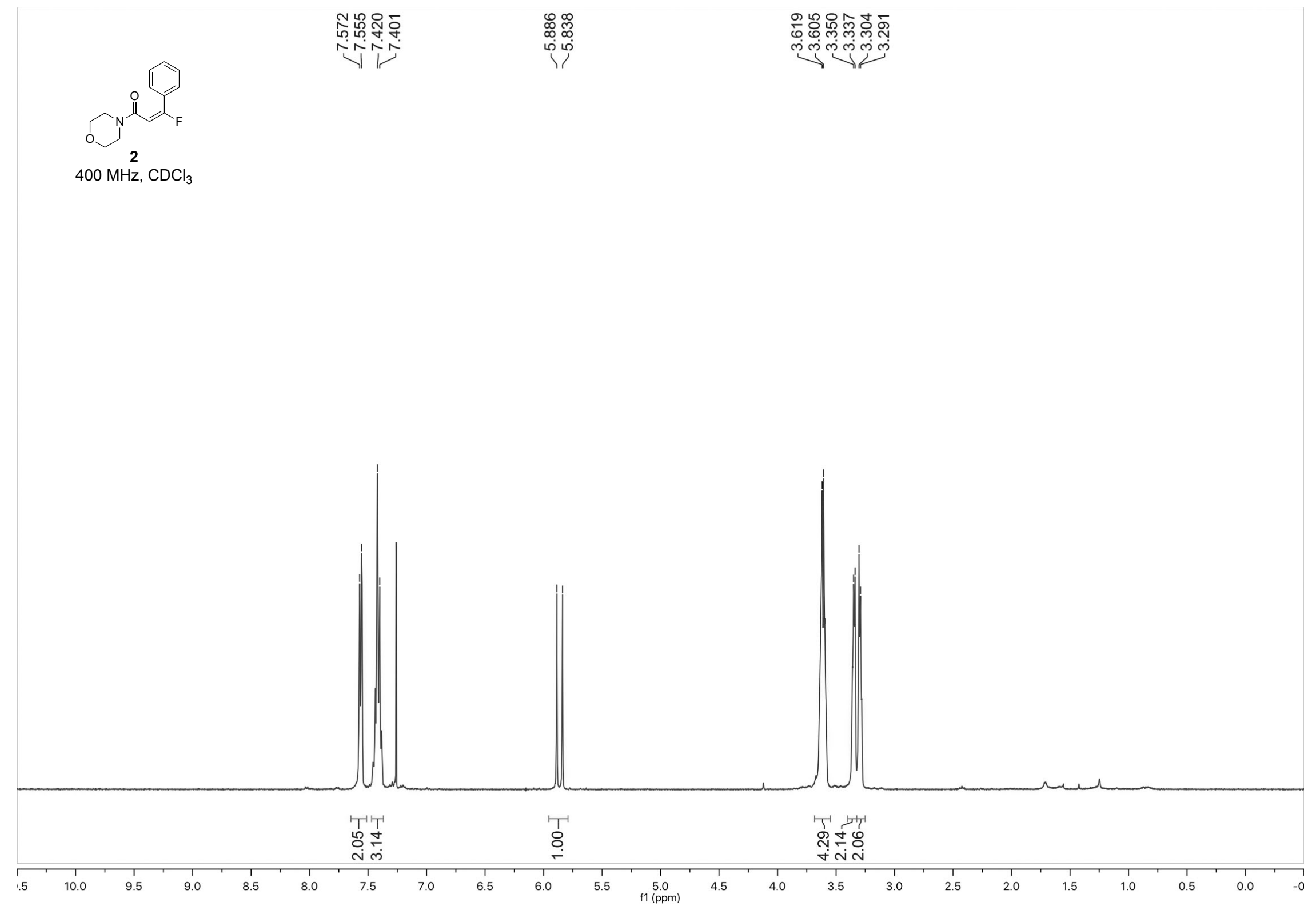




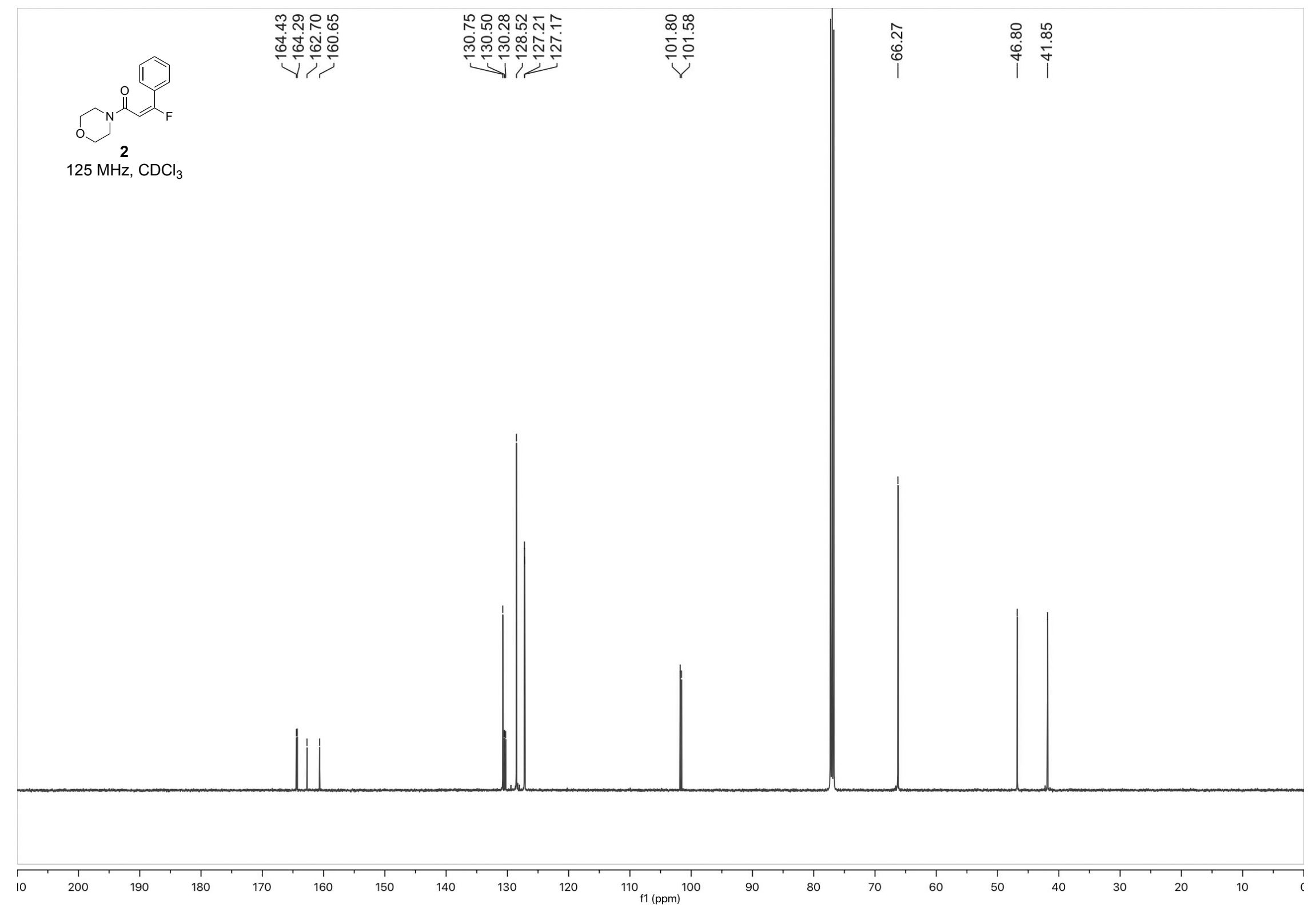




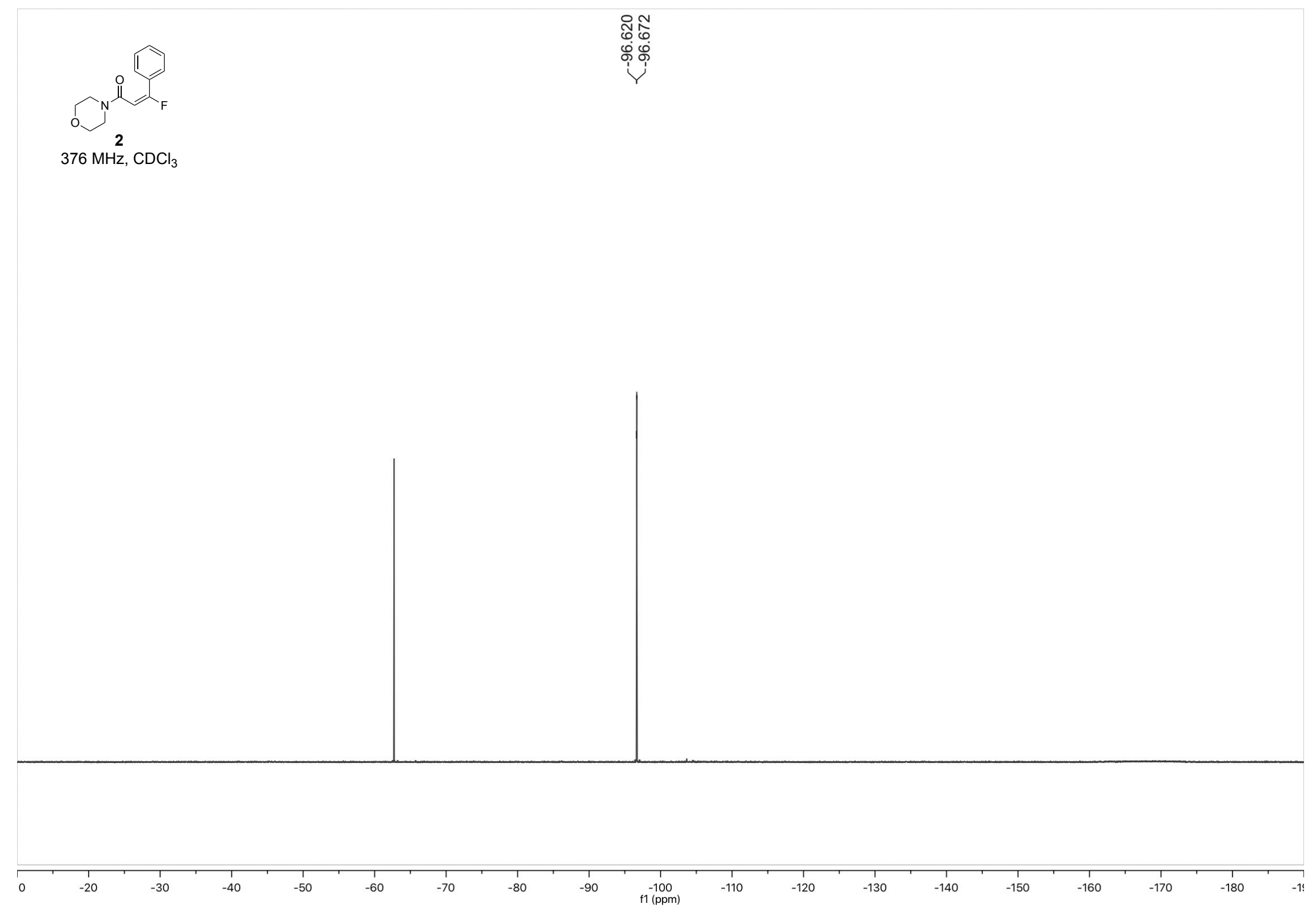




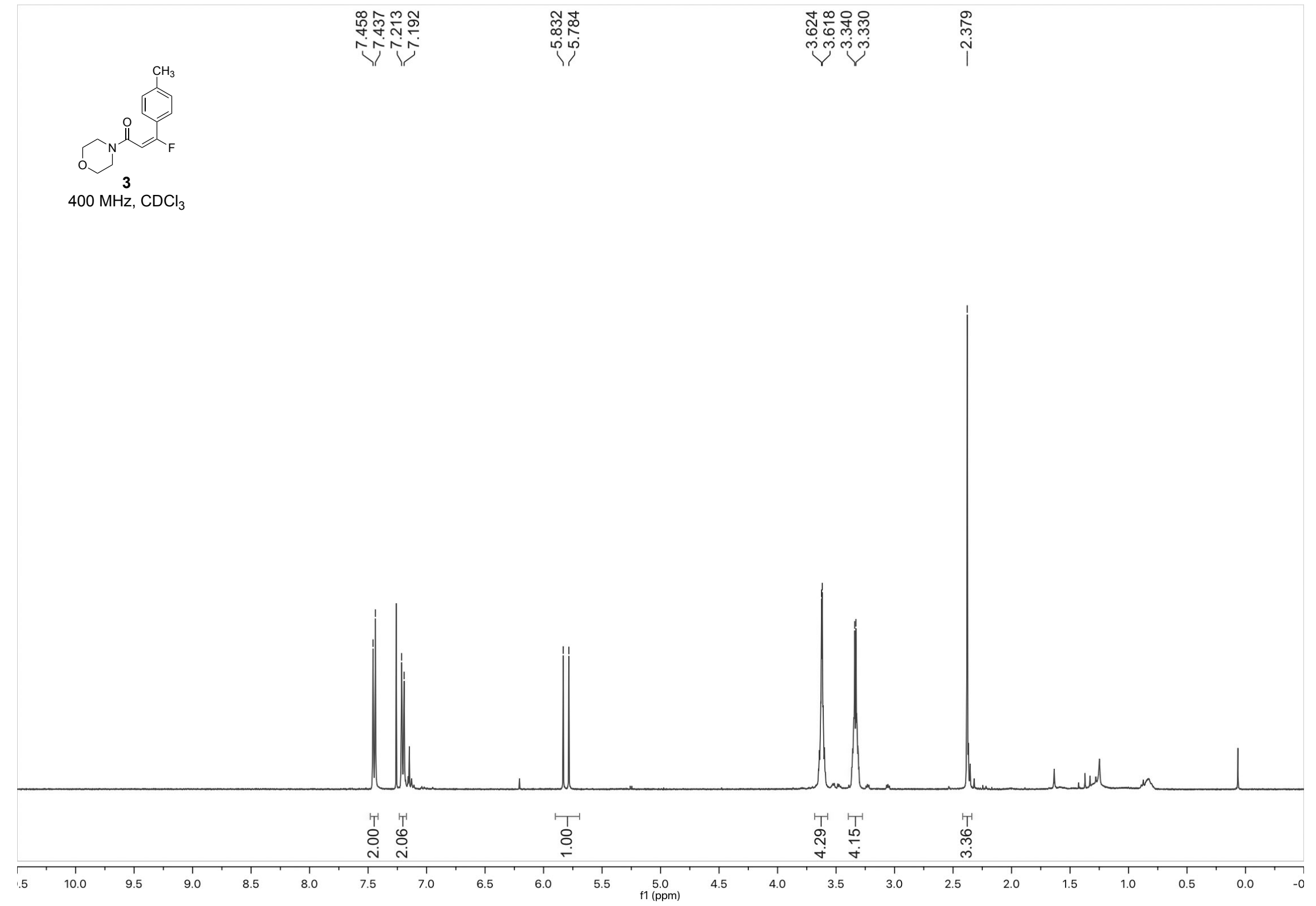



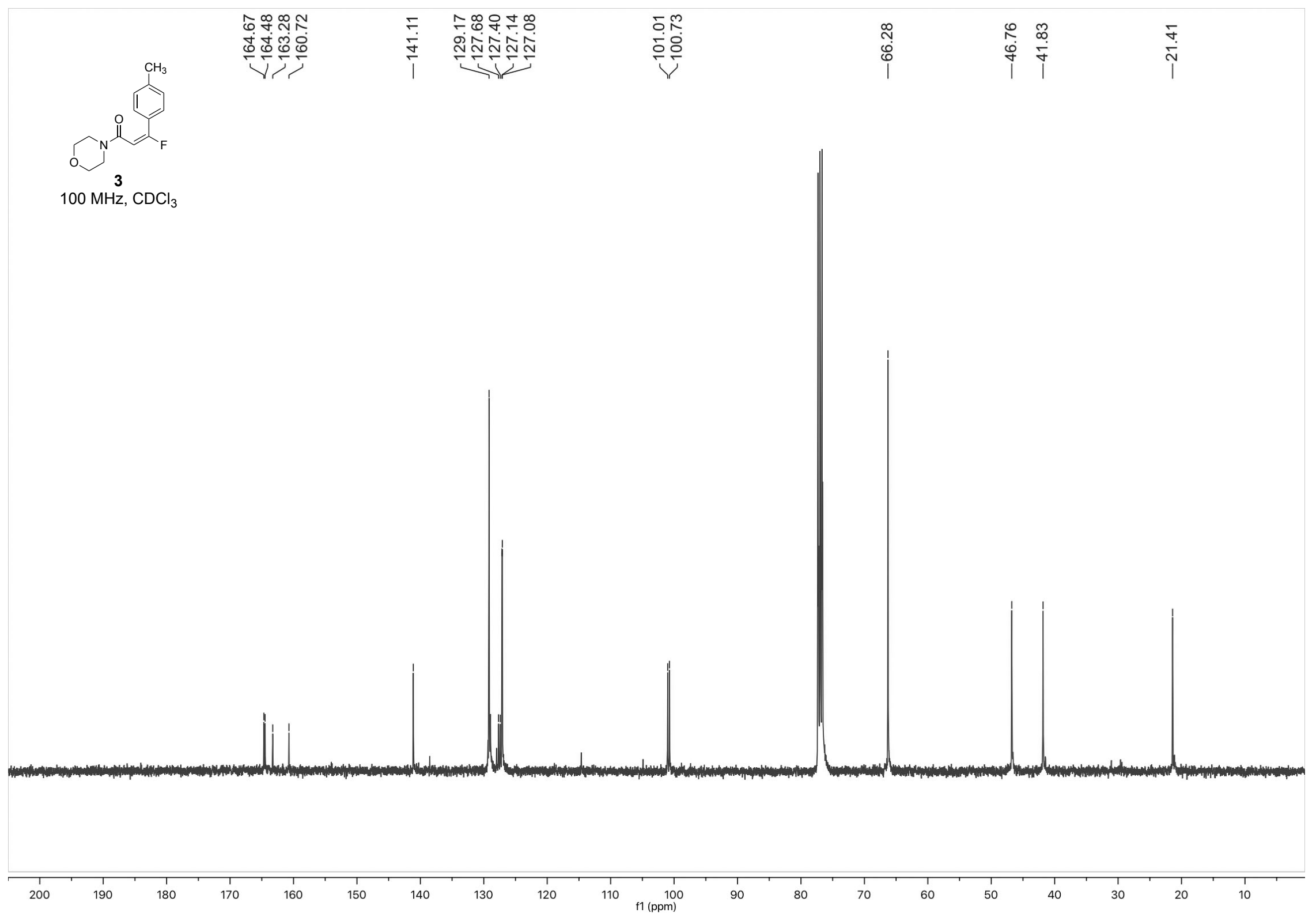


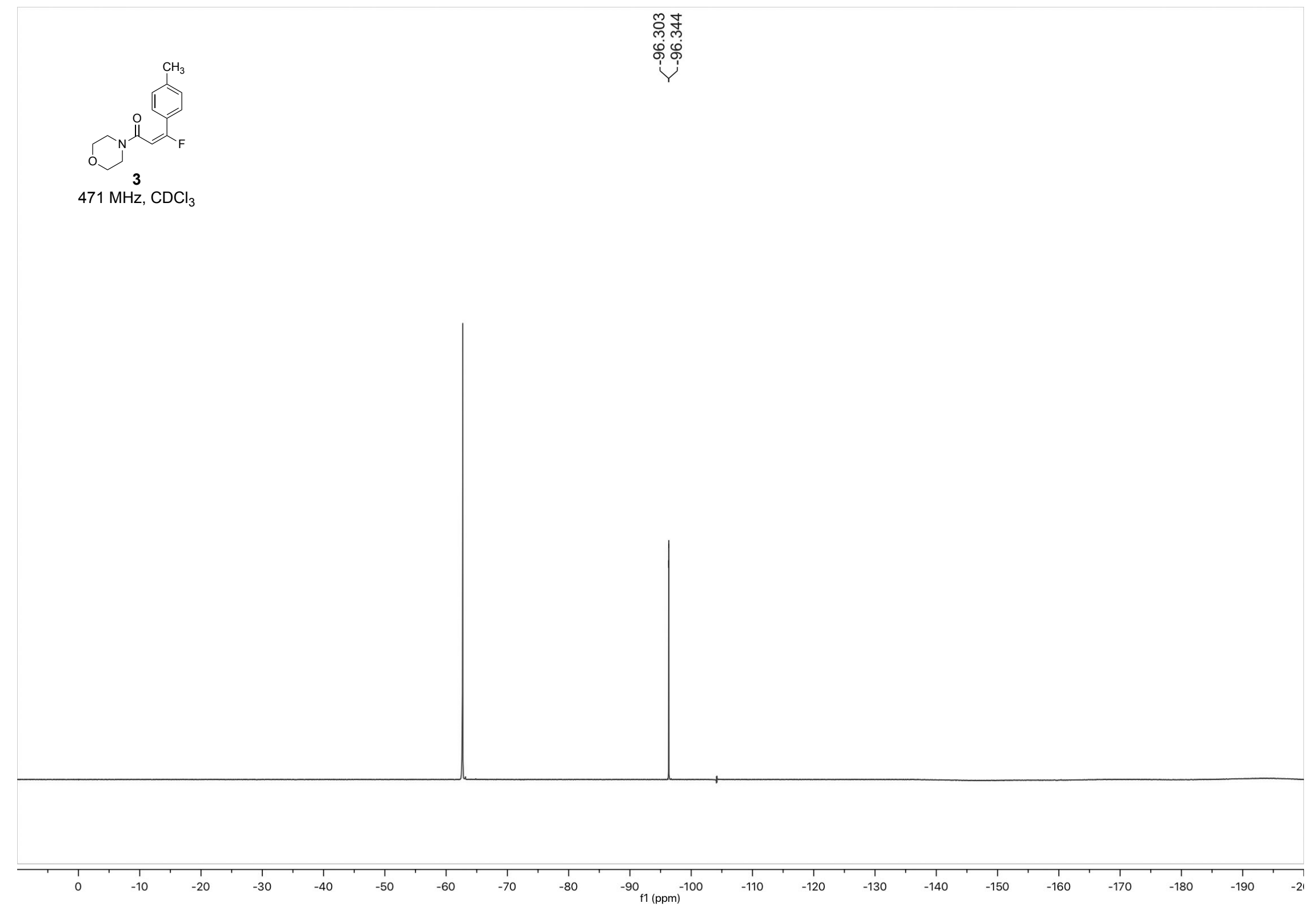




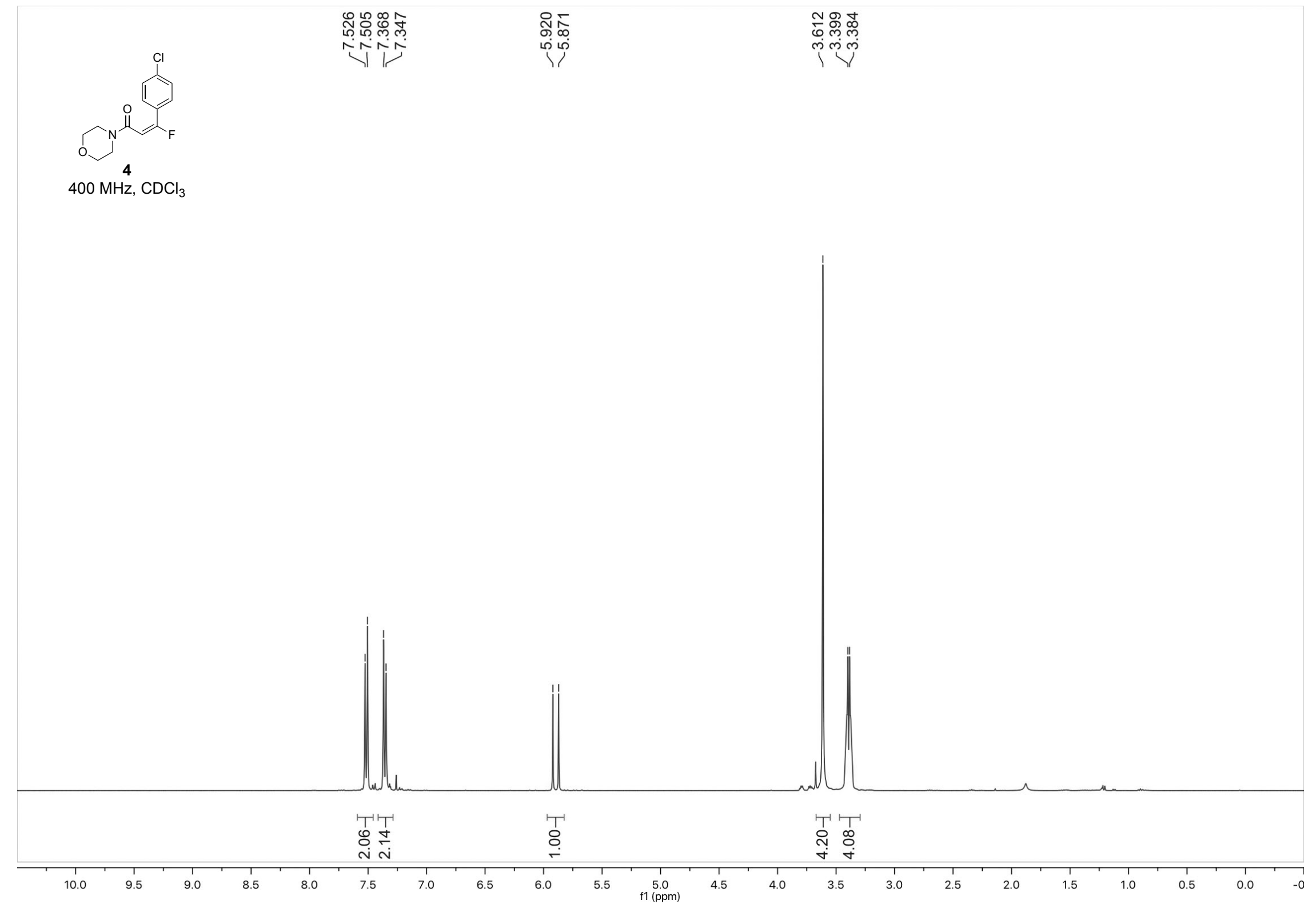




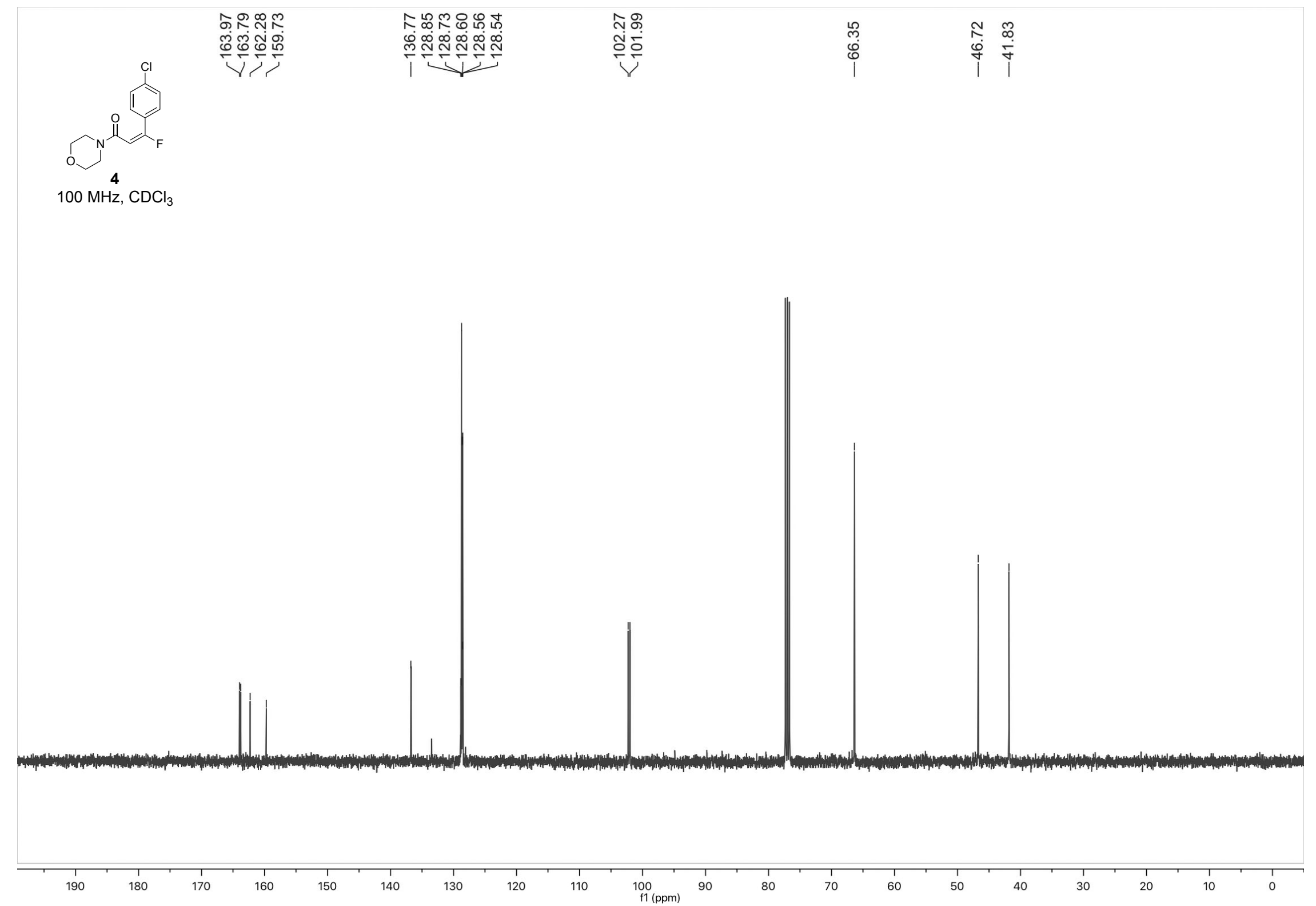




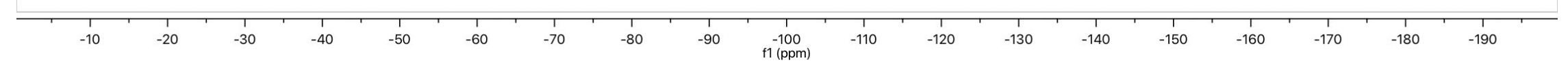




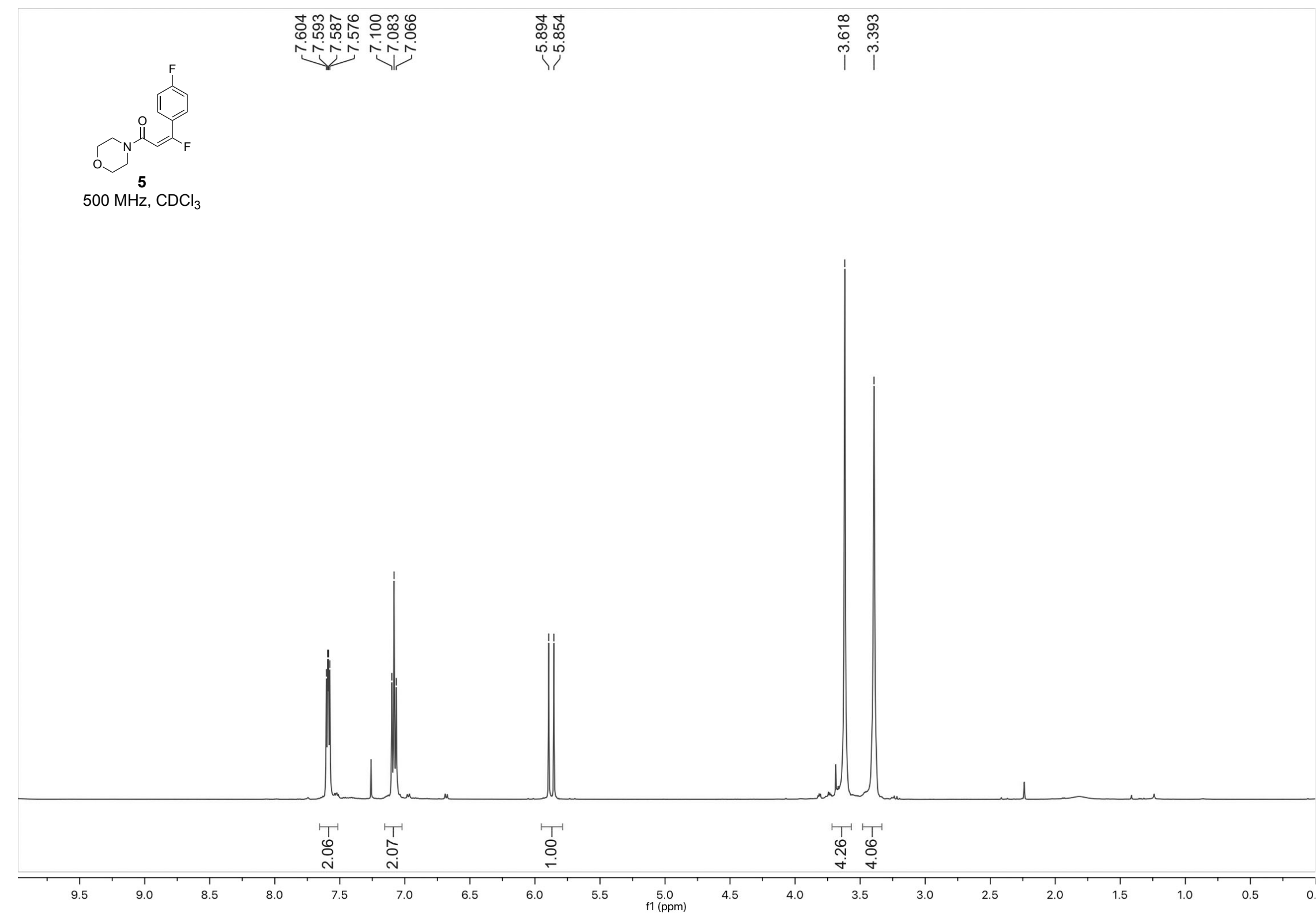




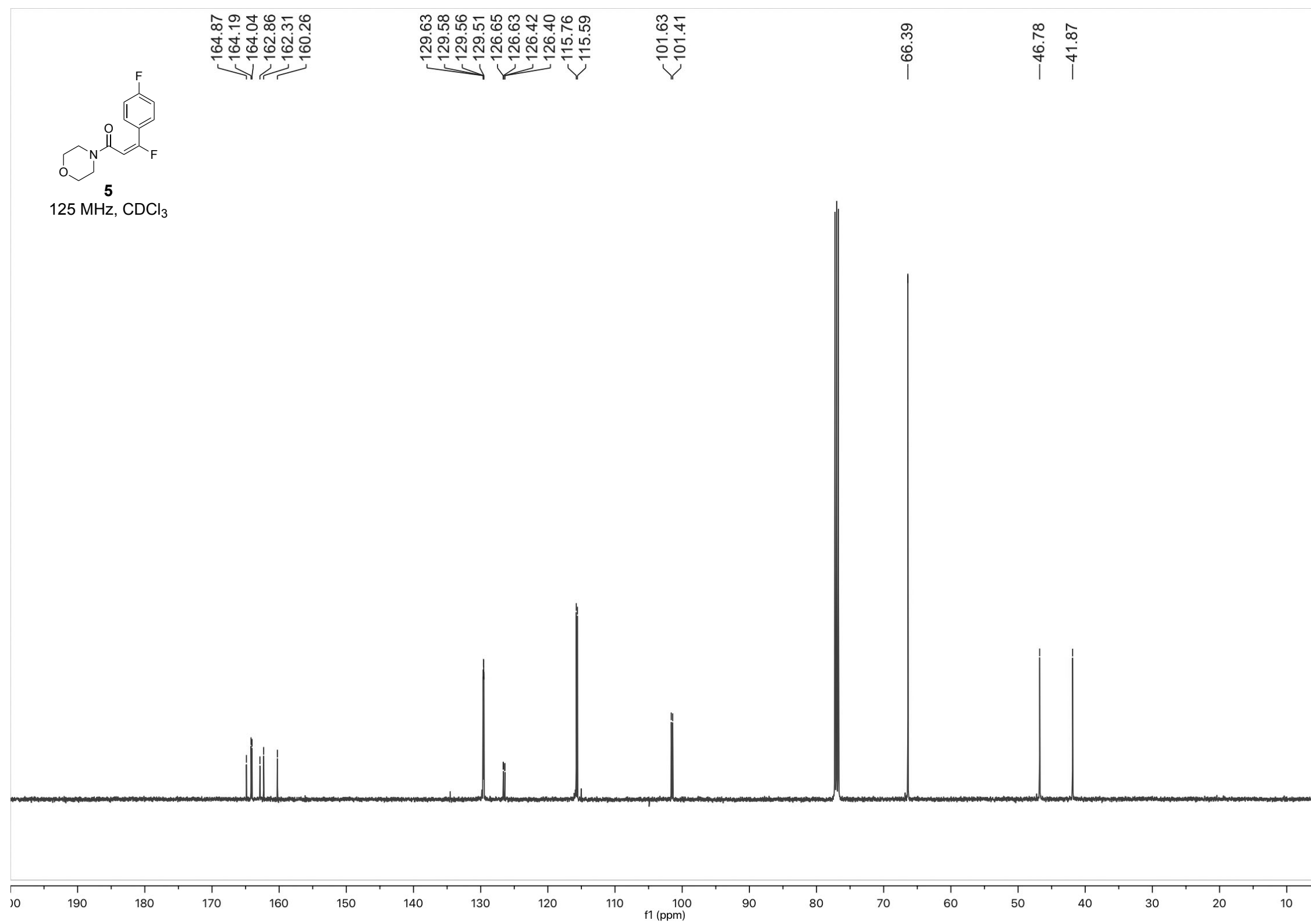

ปั 


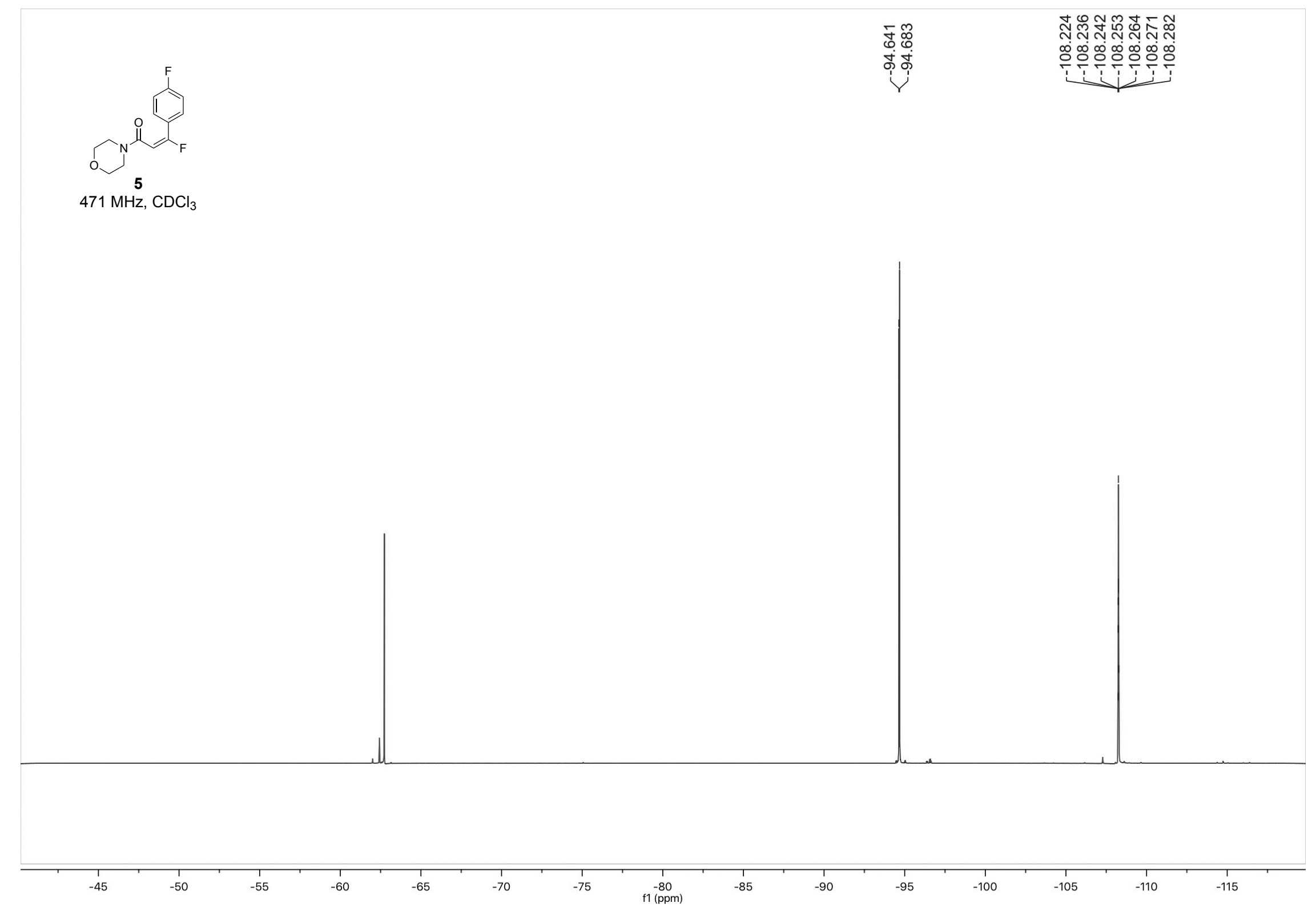




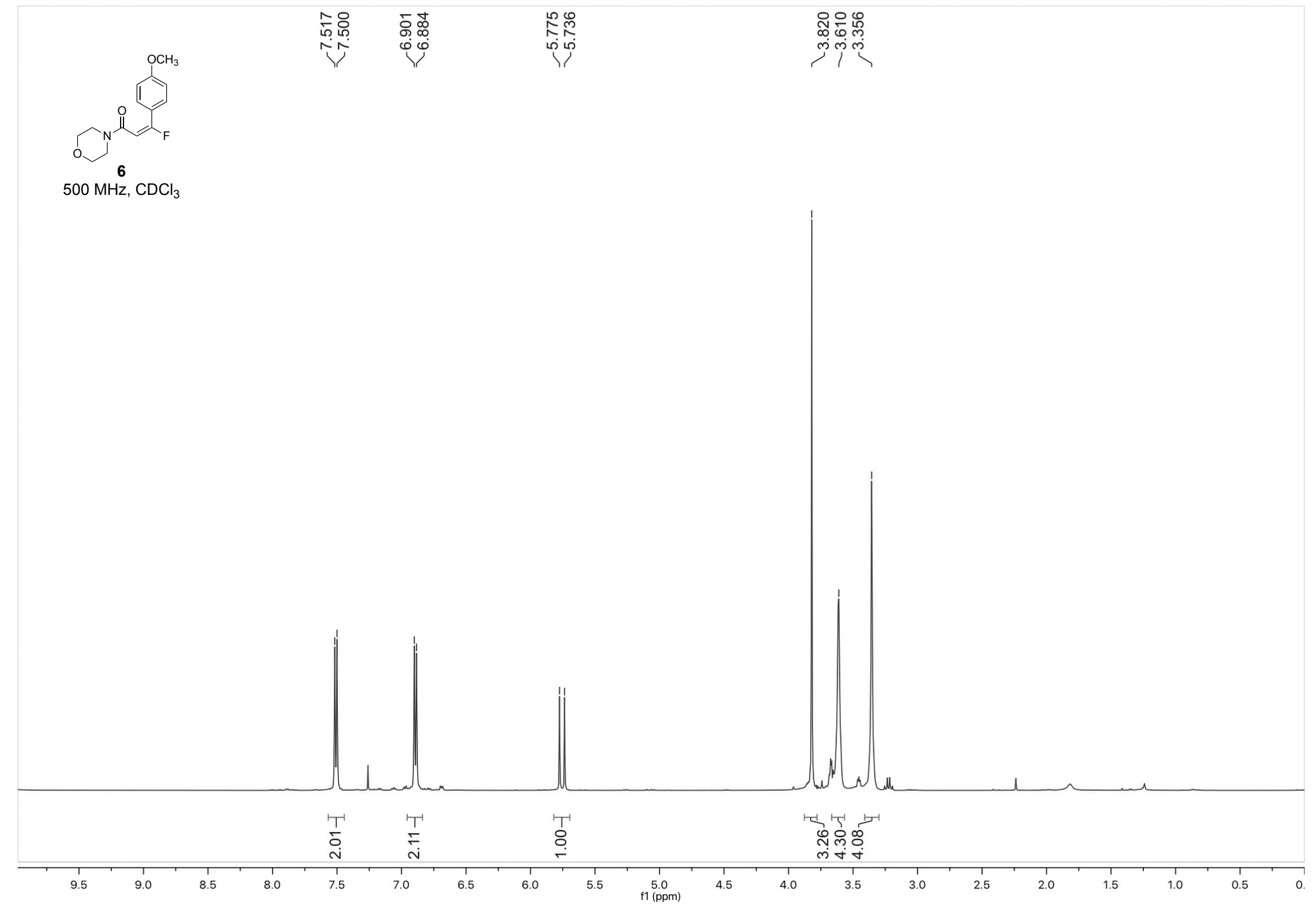




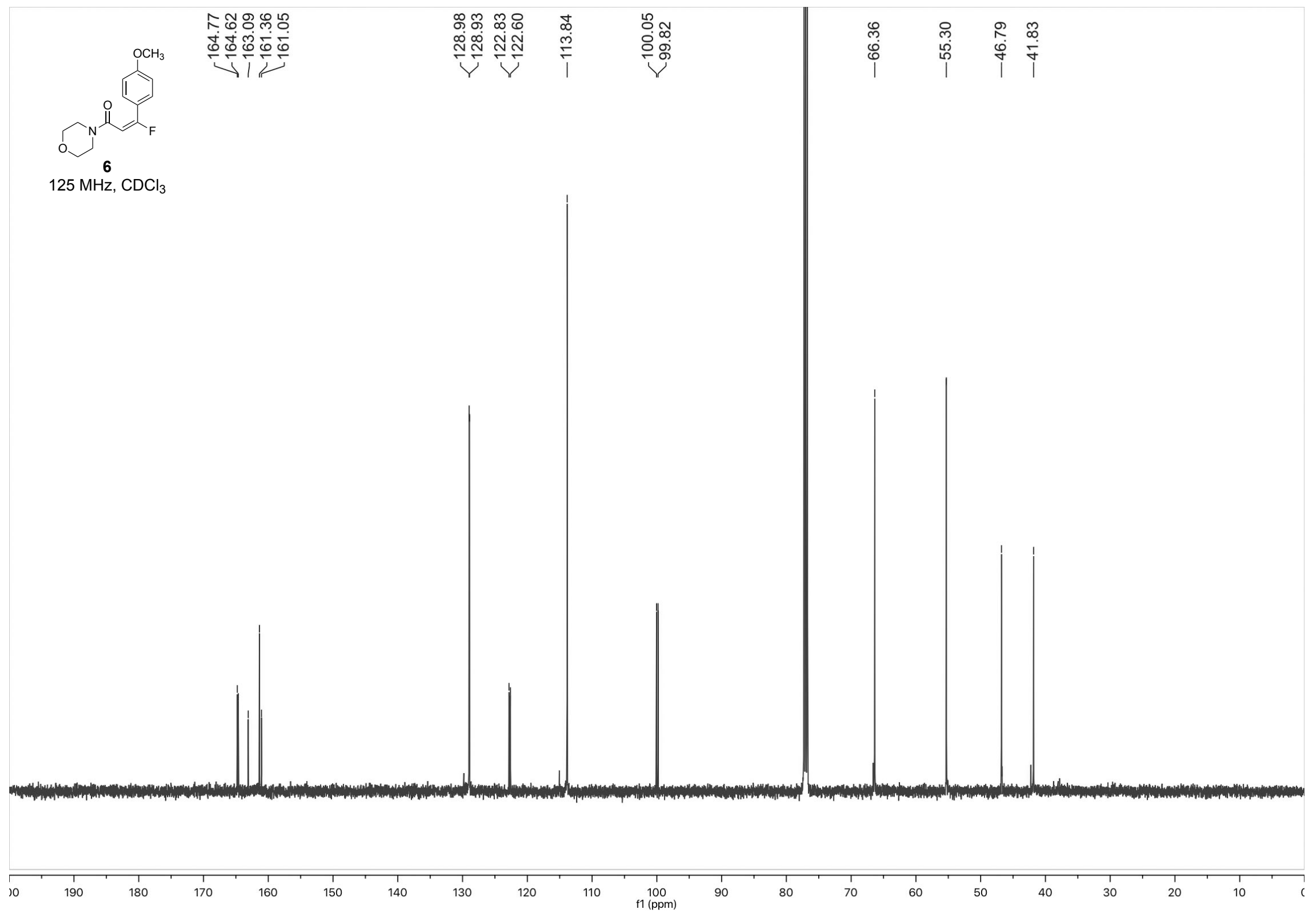




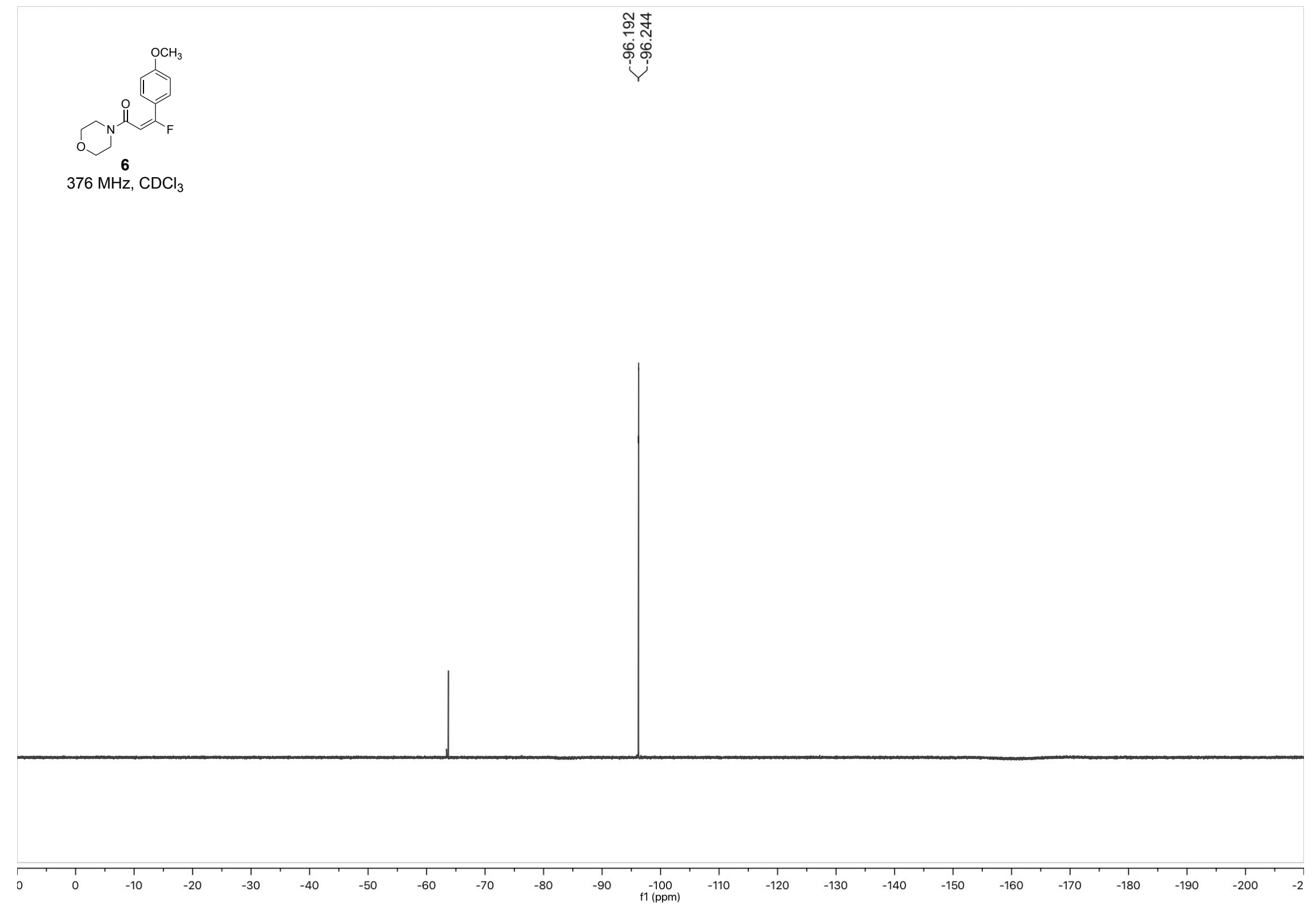




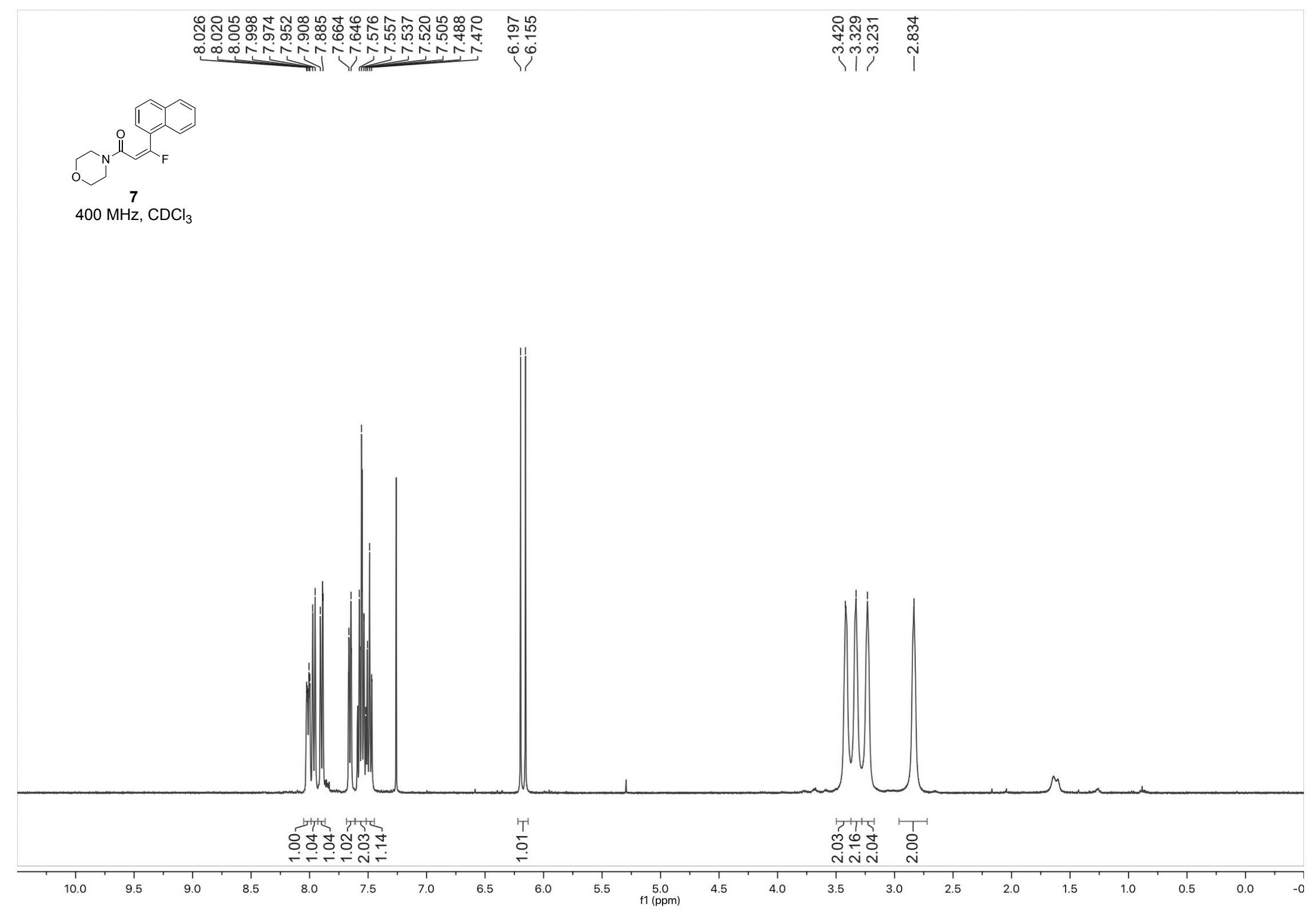




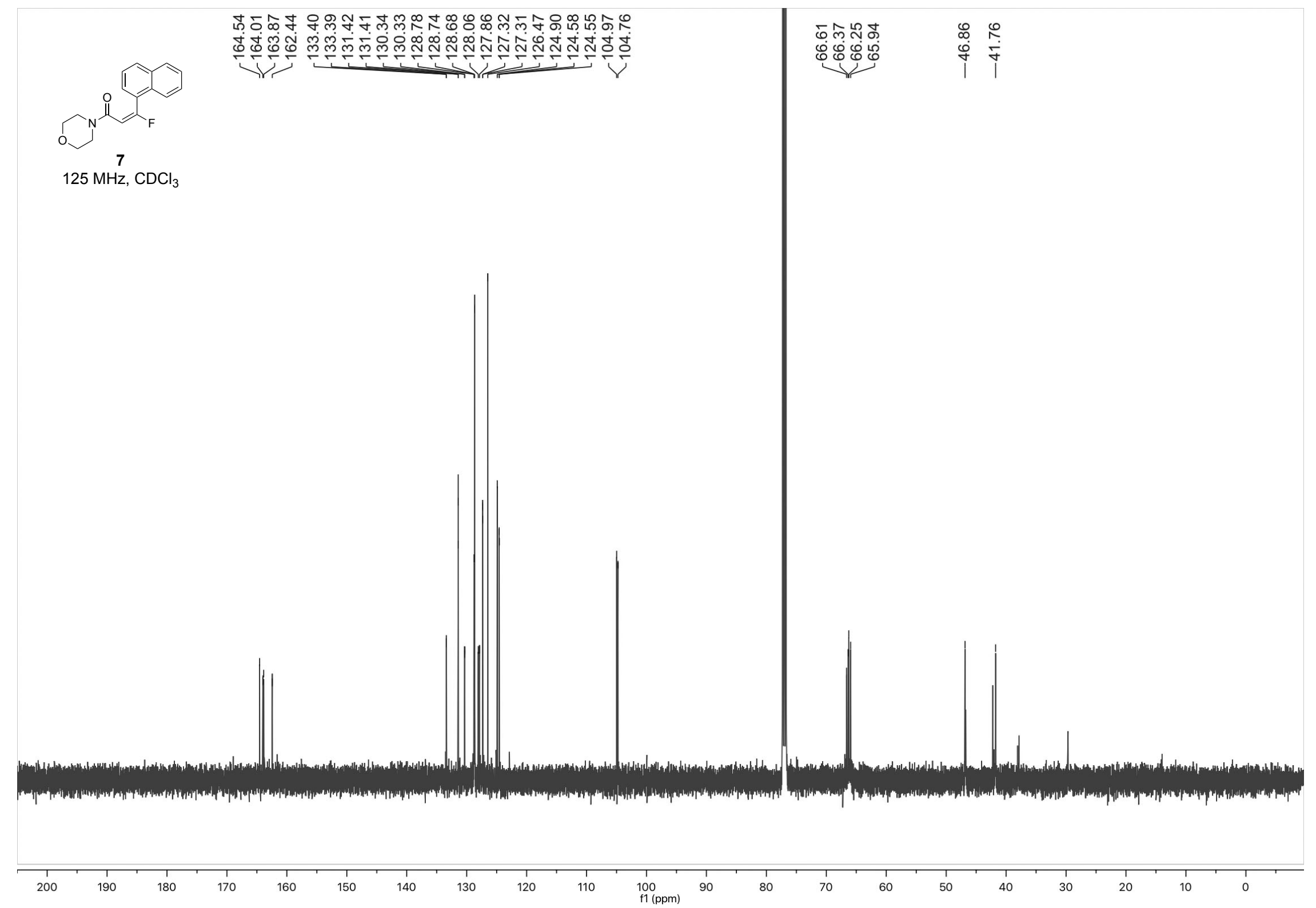




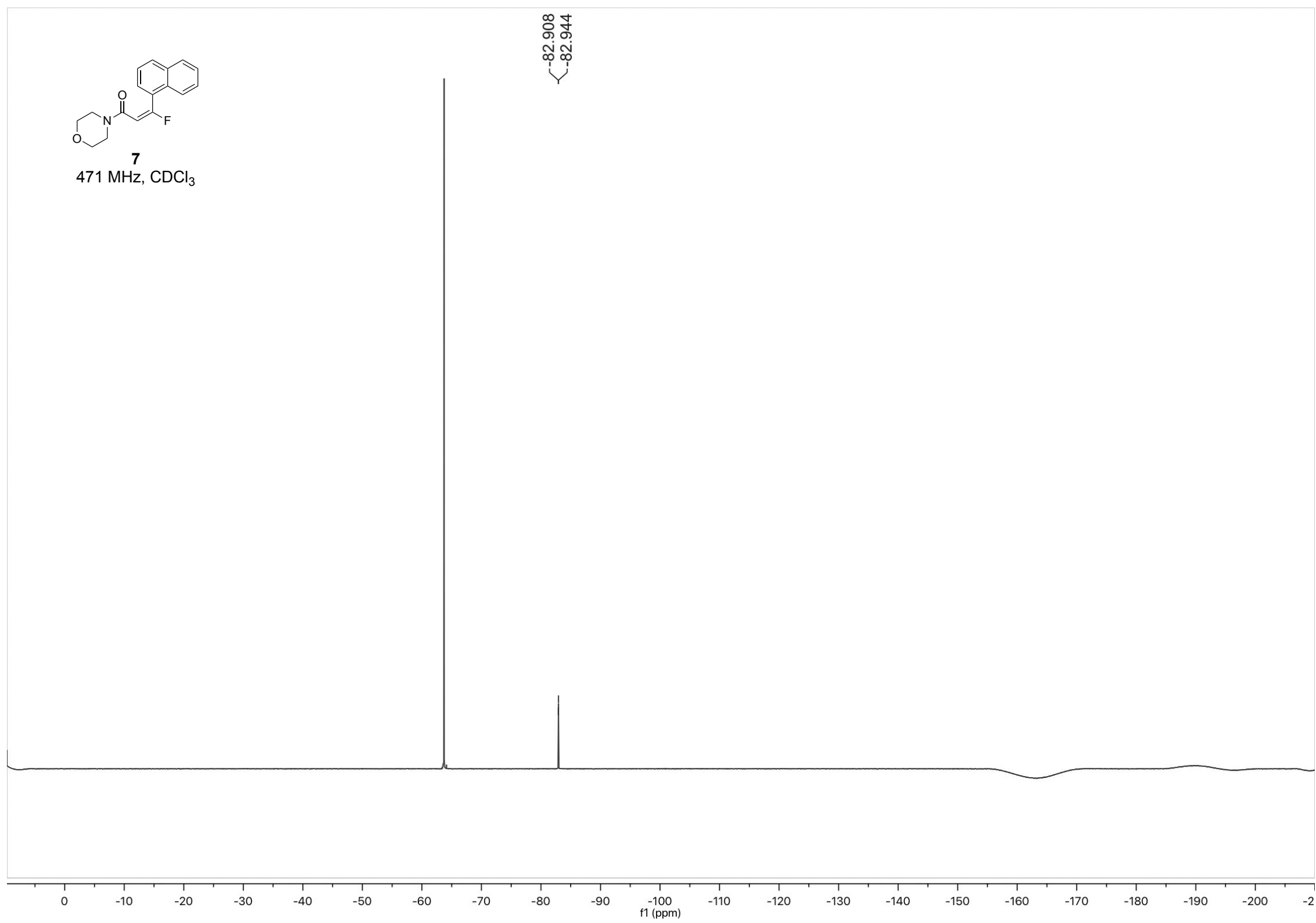

$\bar{n}$ 


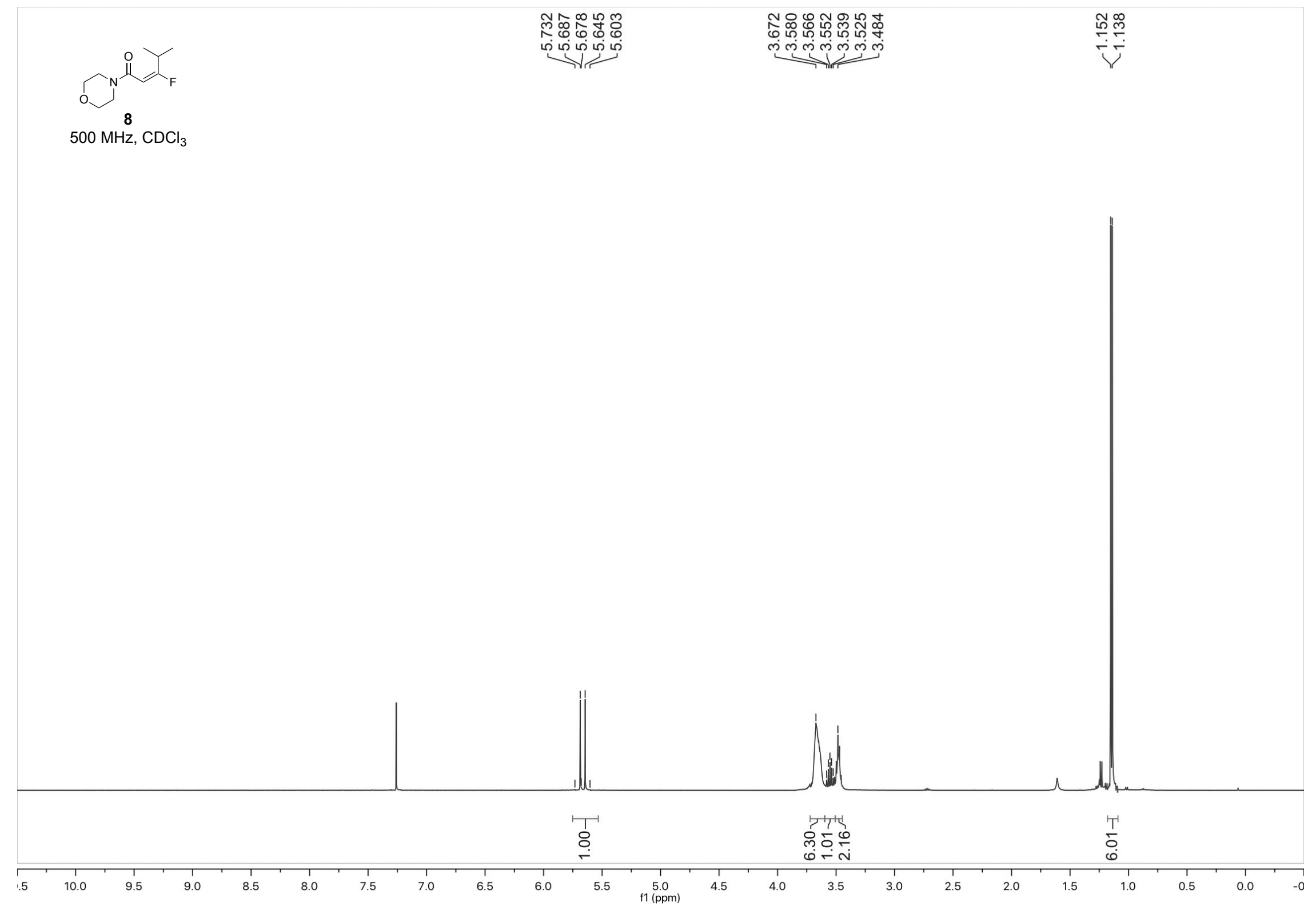




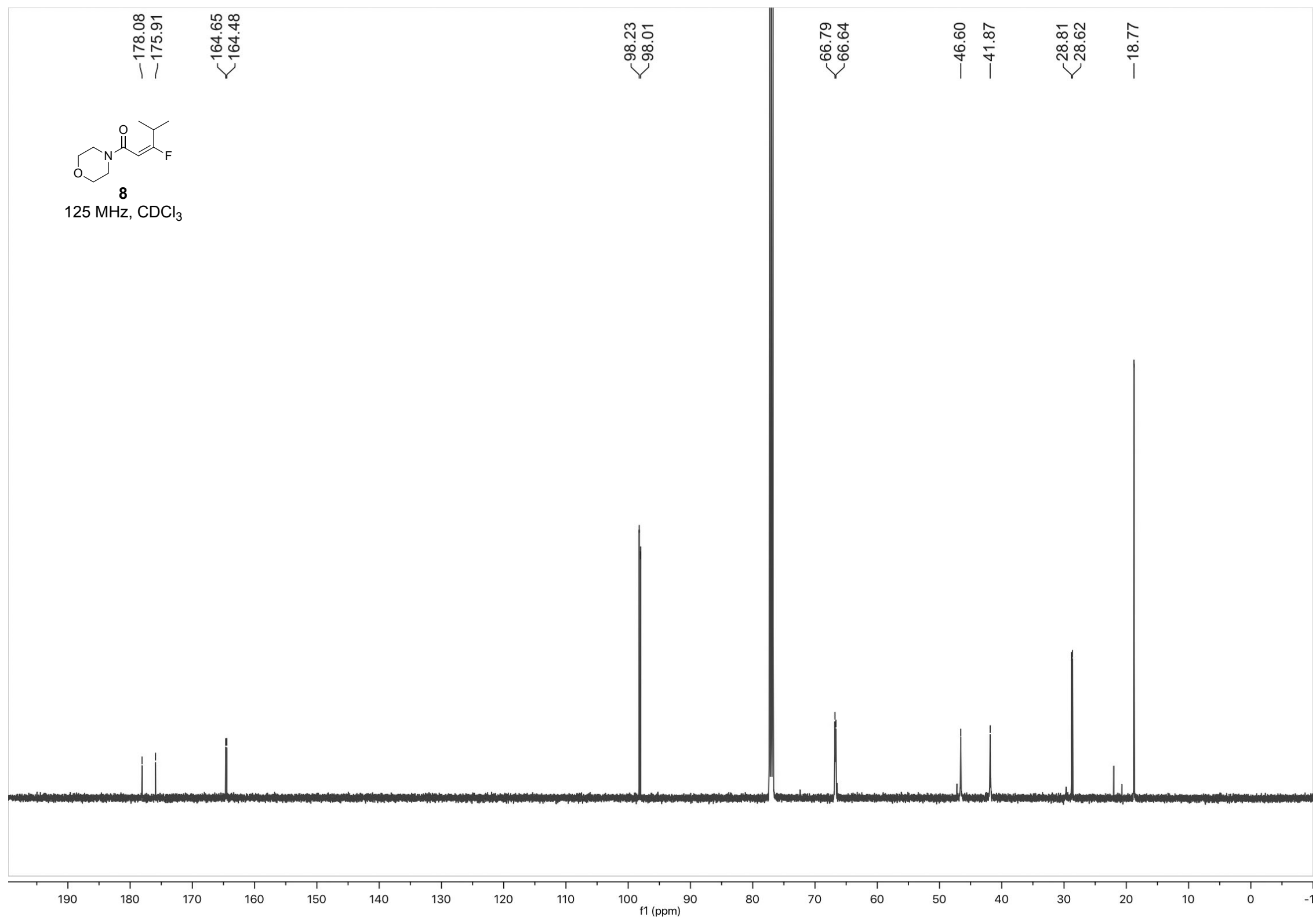




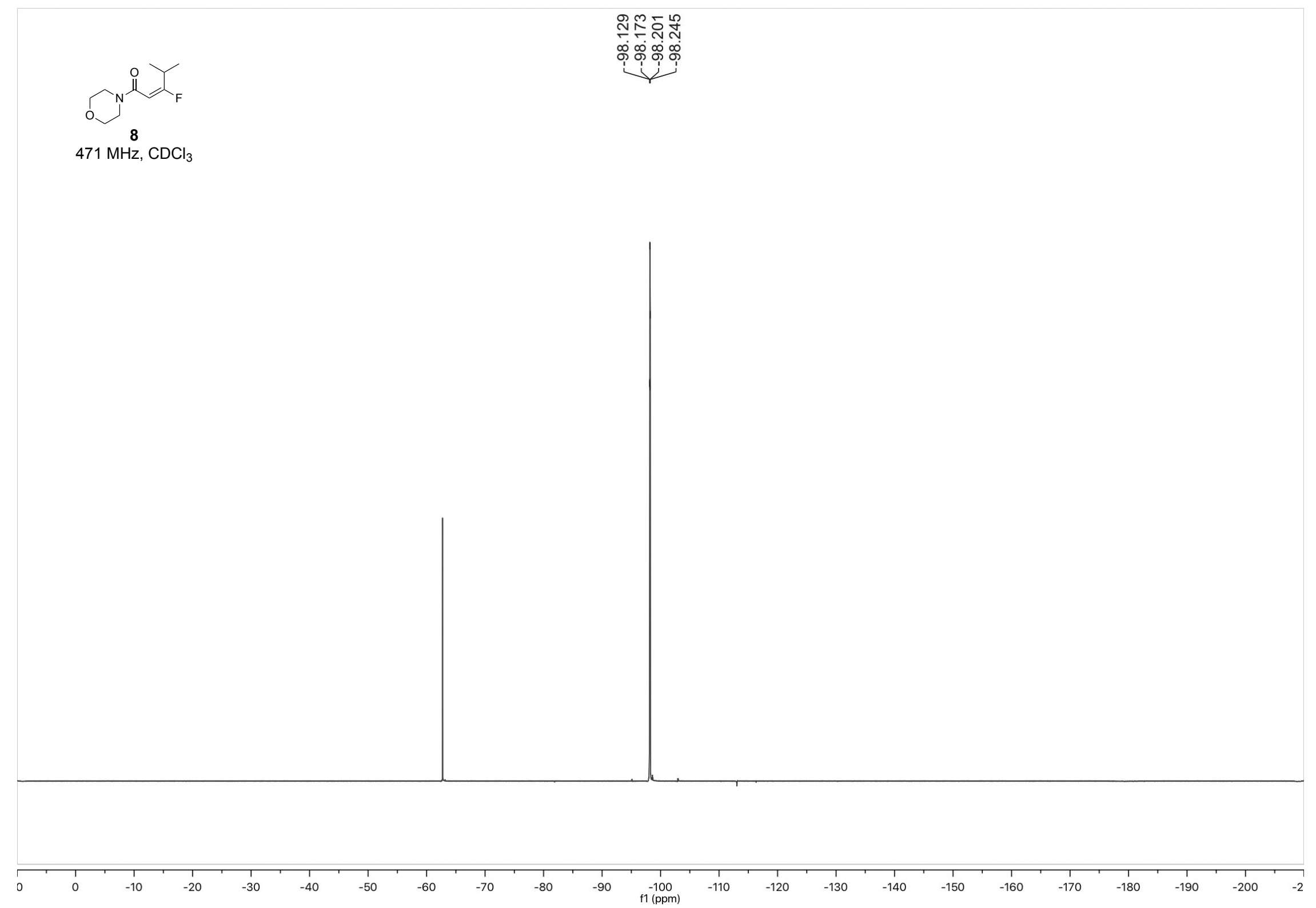




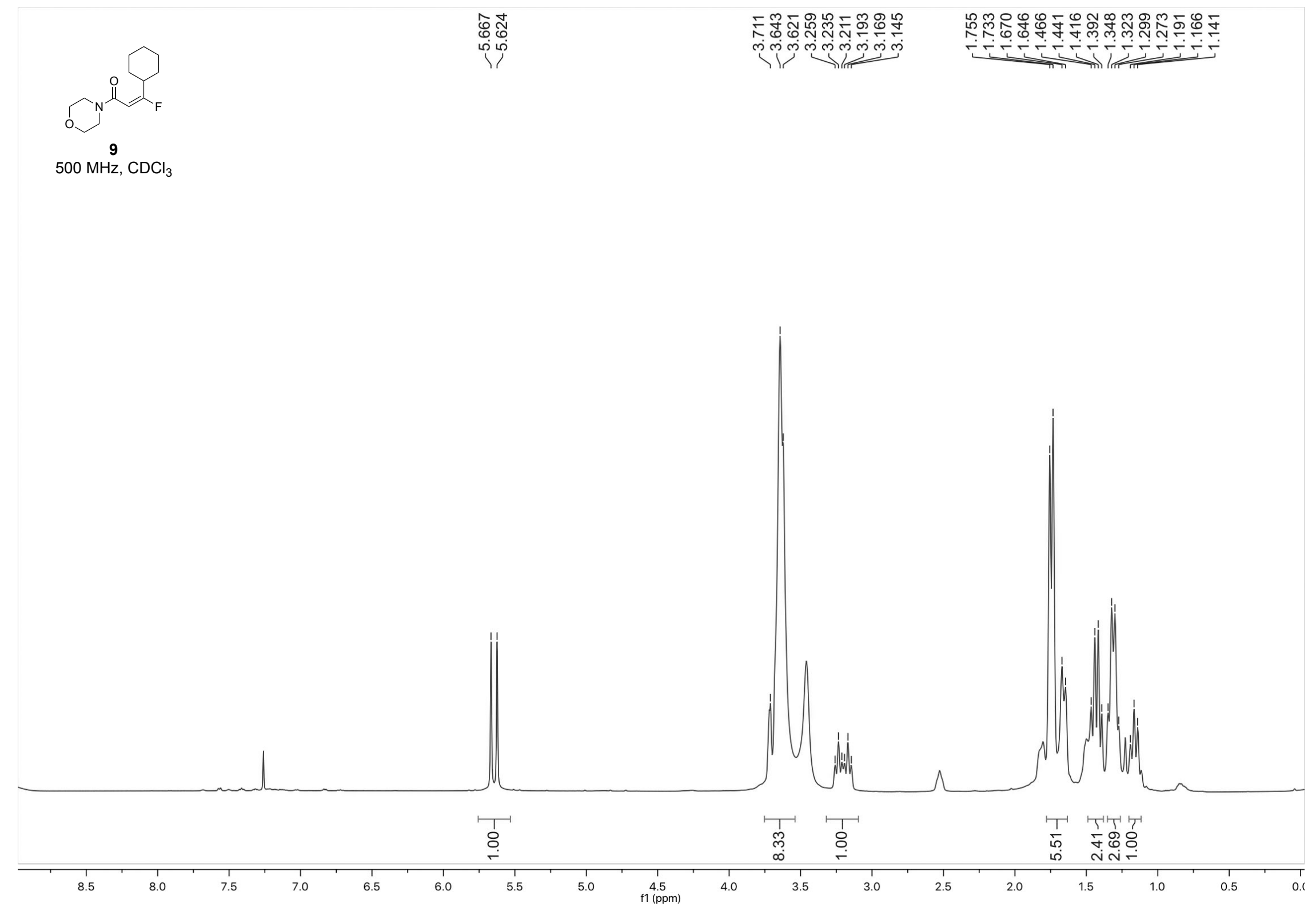




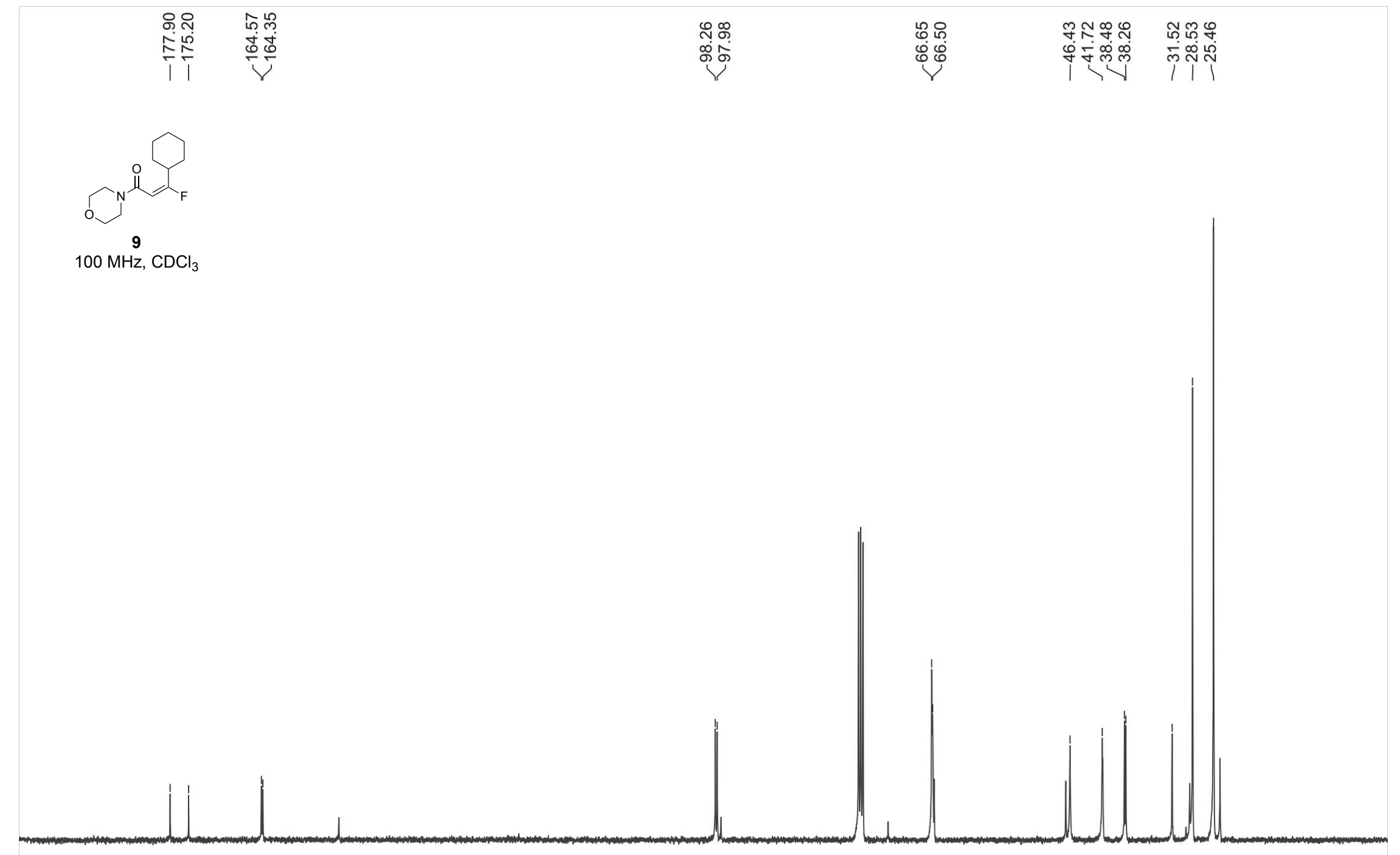

ñ$$
10
$$ 


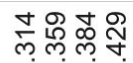

$\overbrace{}^{9}$

象

$471 \mathrm{MHz}, \mathrm{CDCl}_{3}$

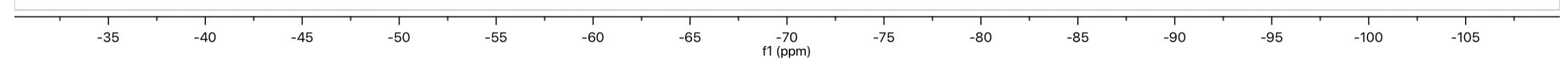




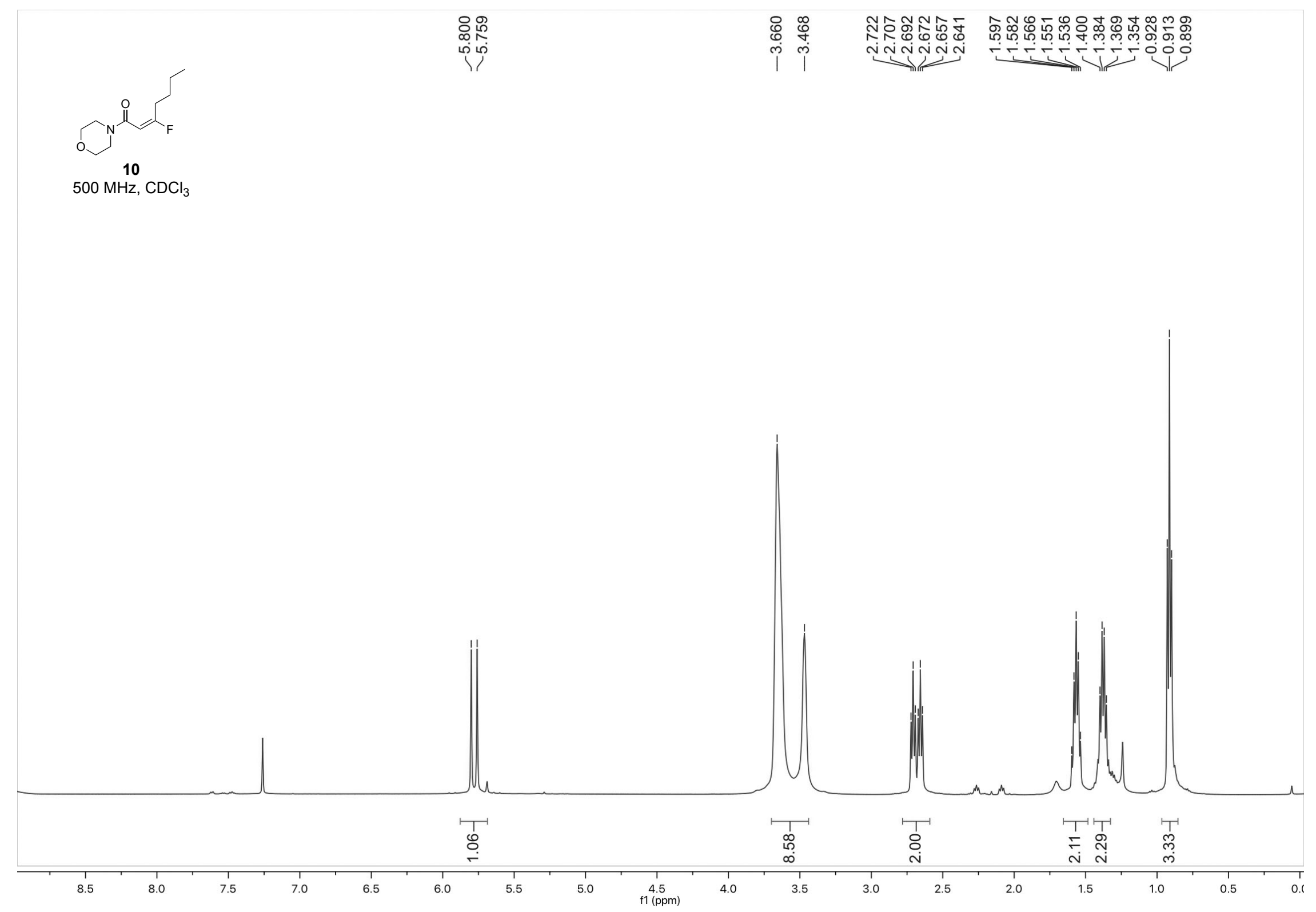




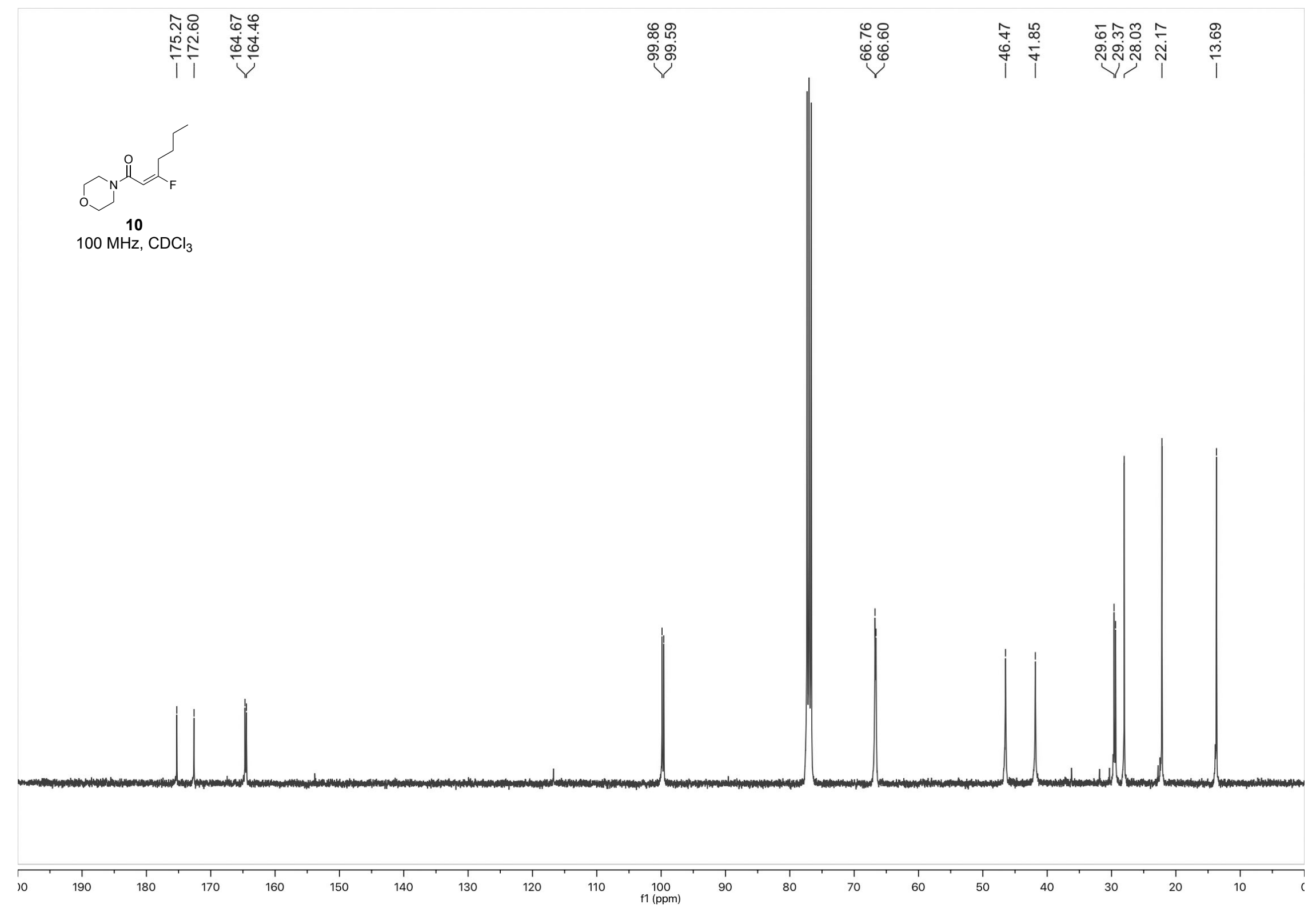

ले 

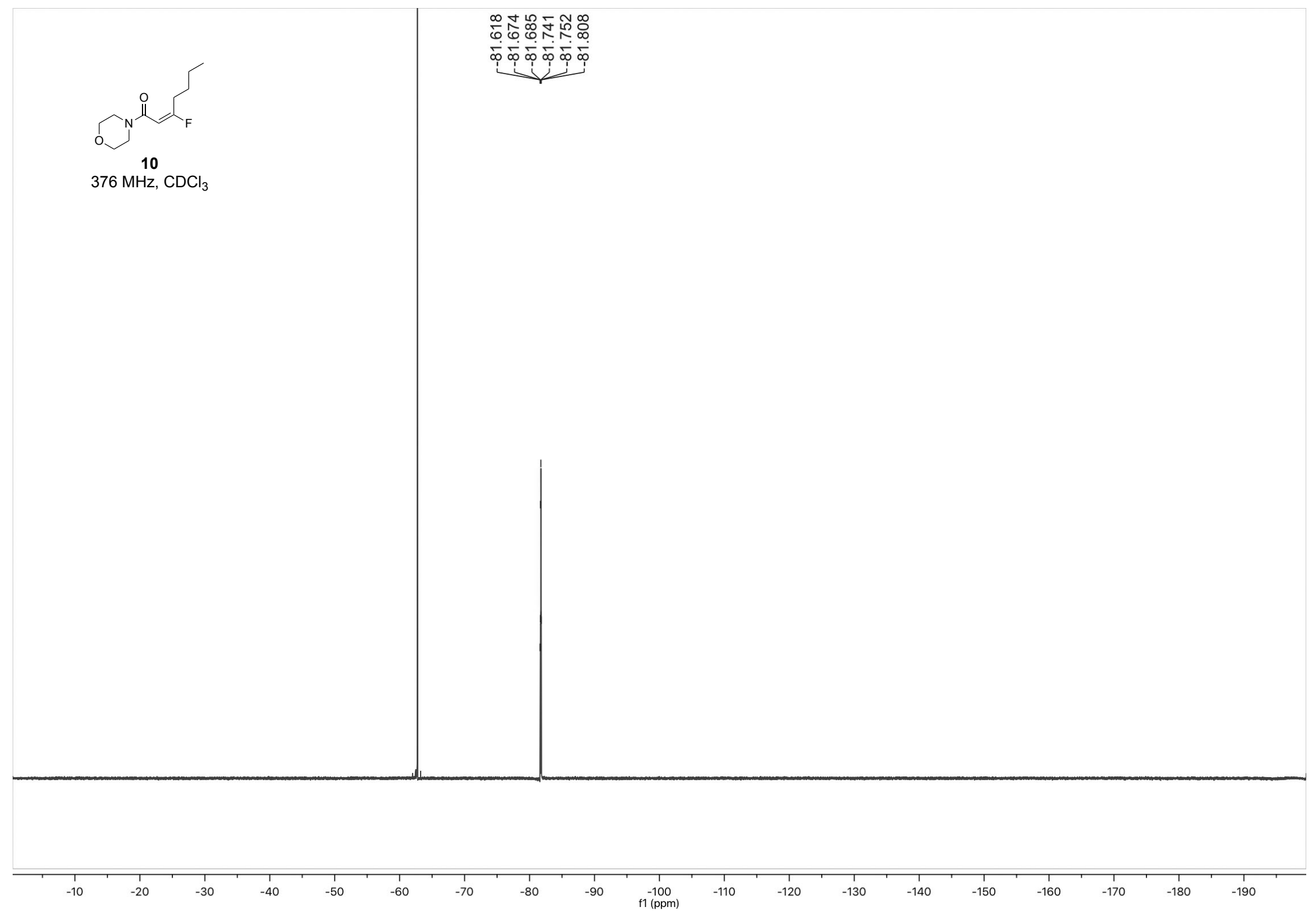


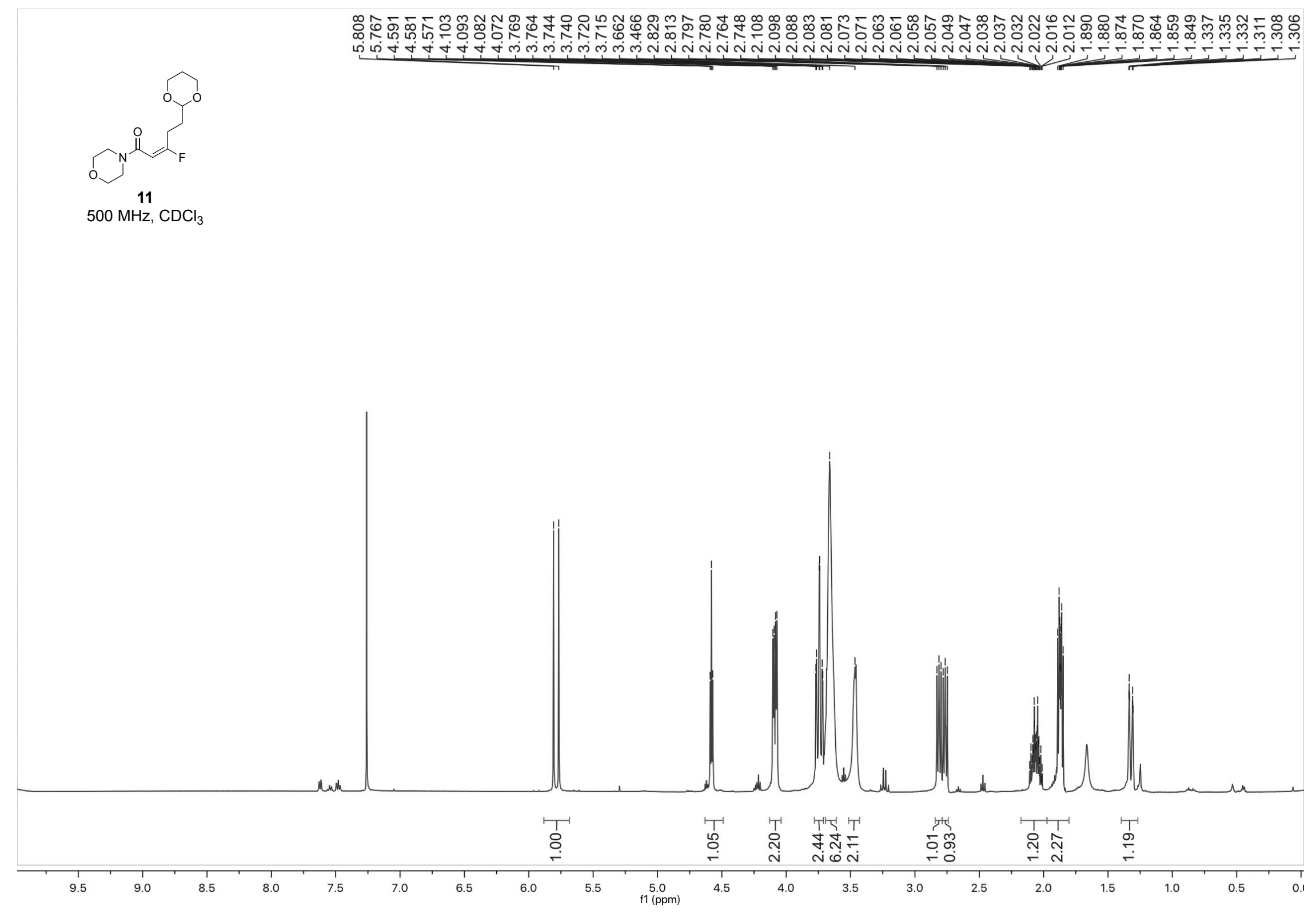




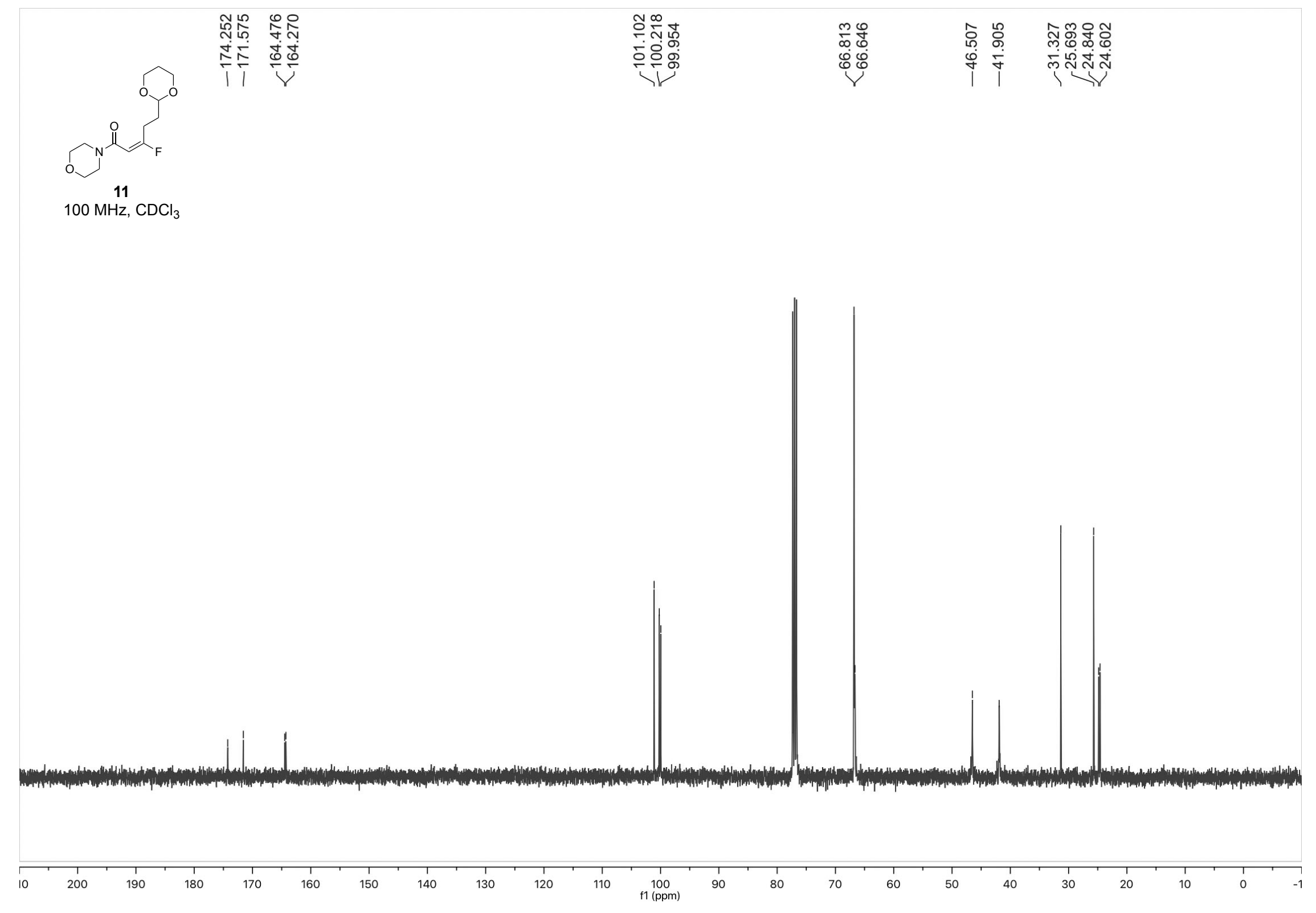




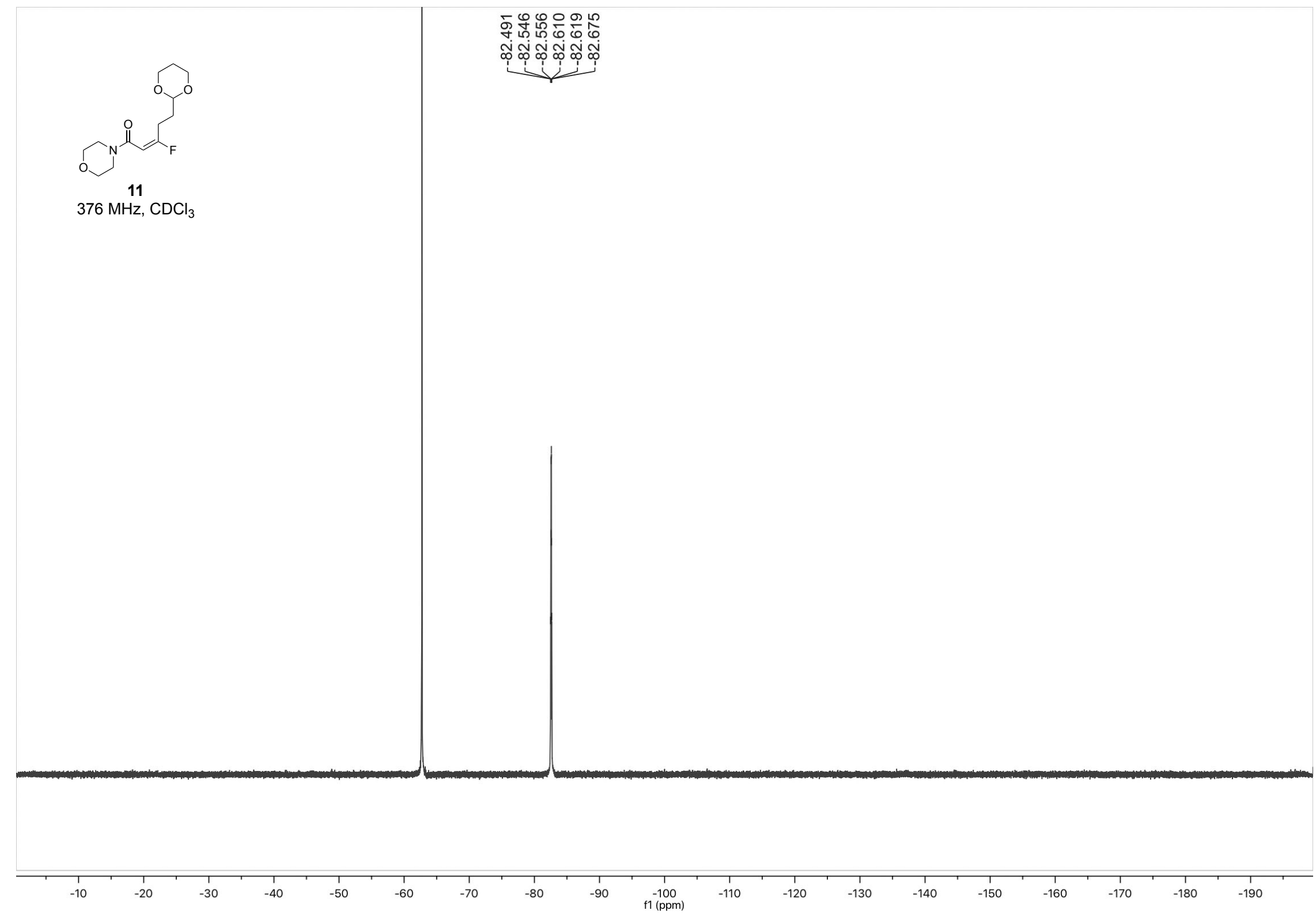




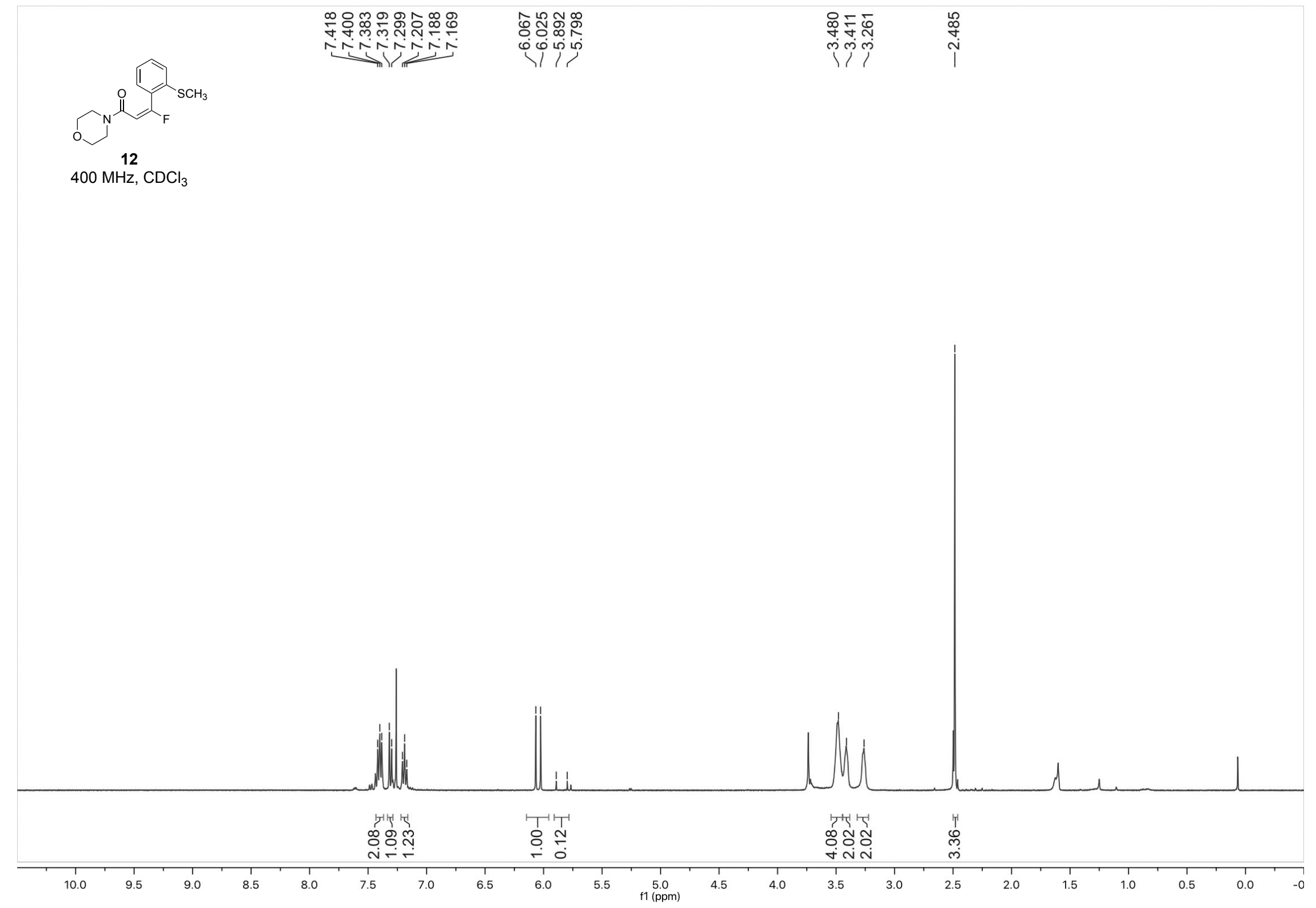



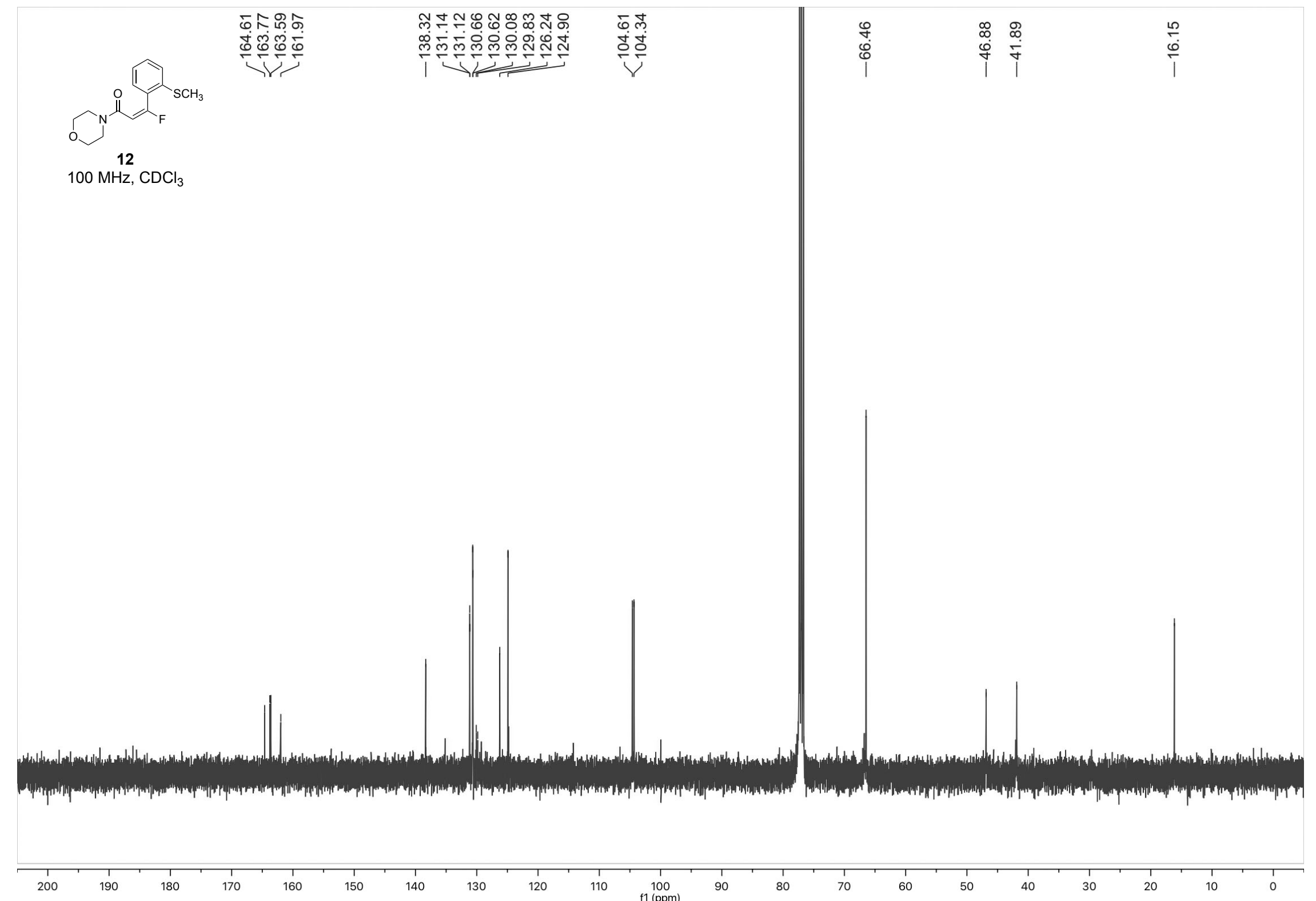


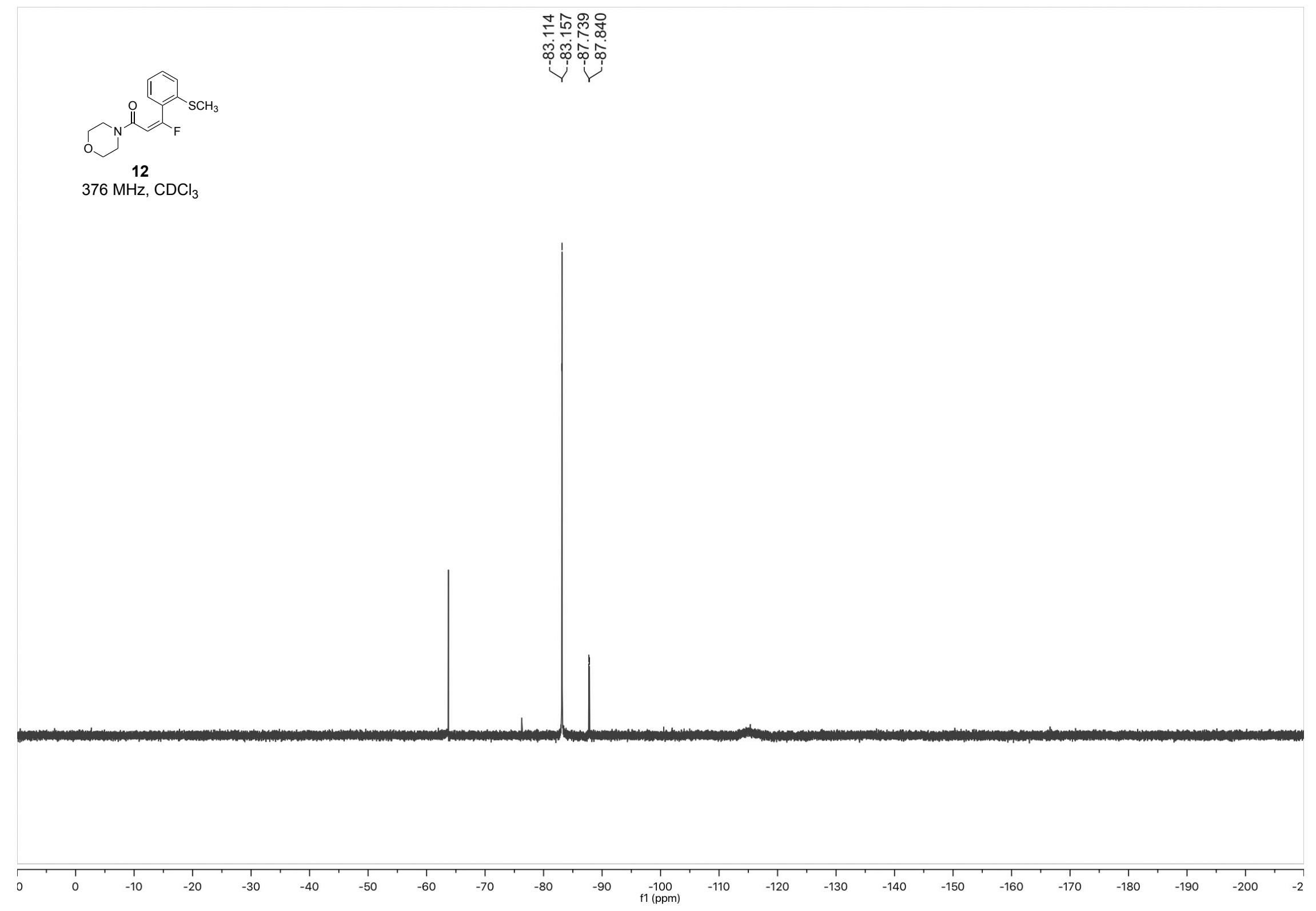




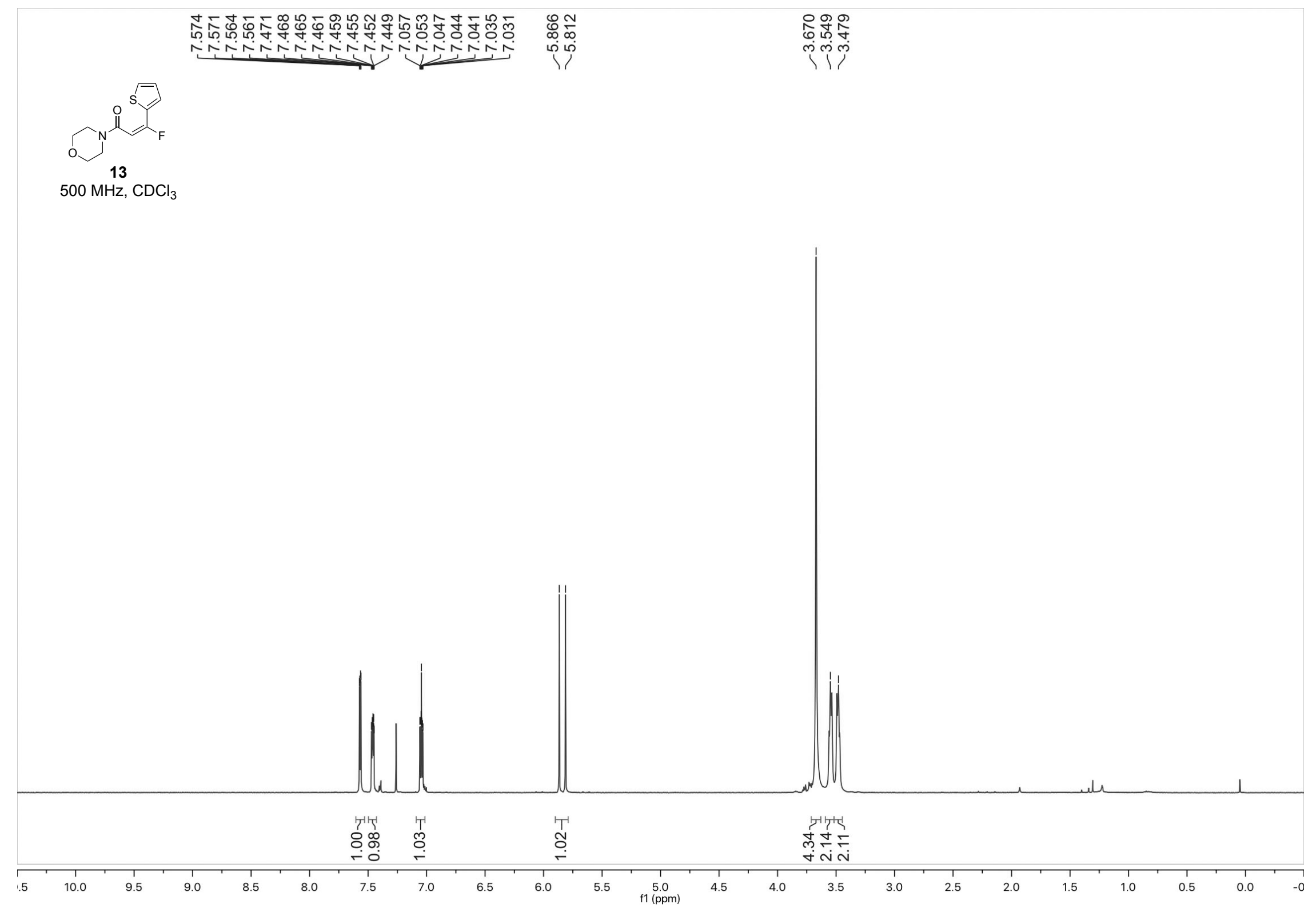




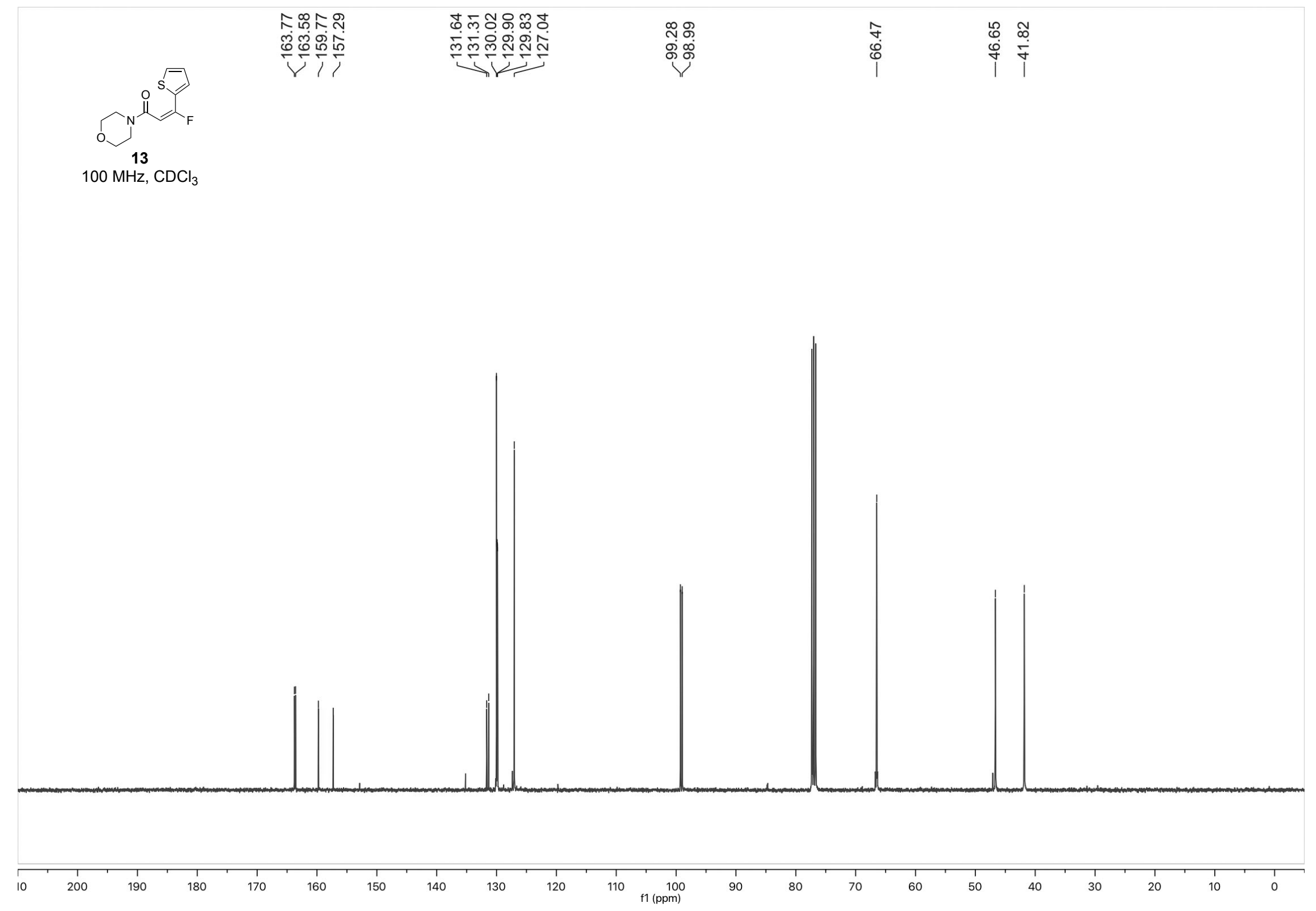




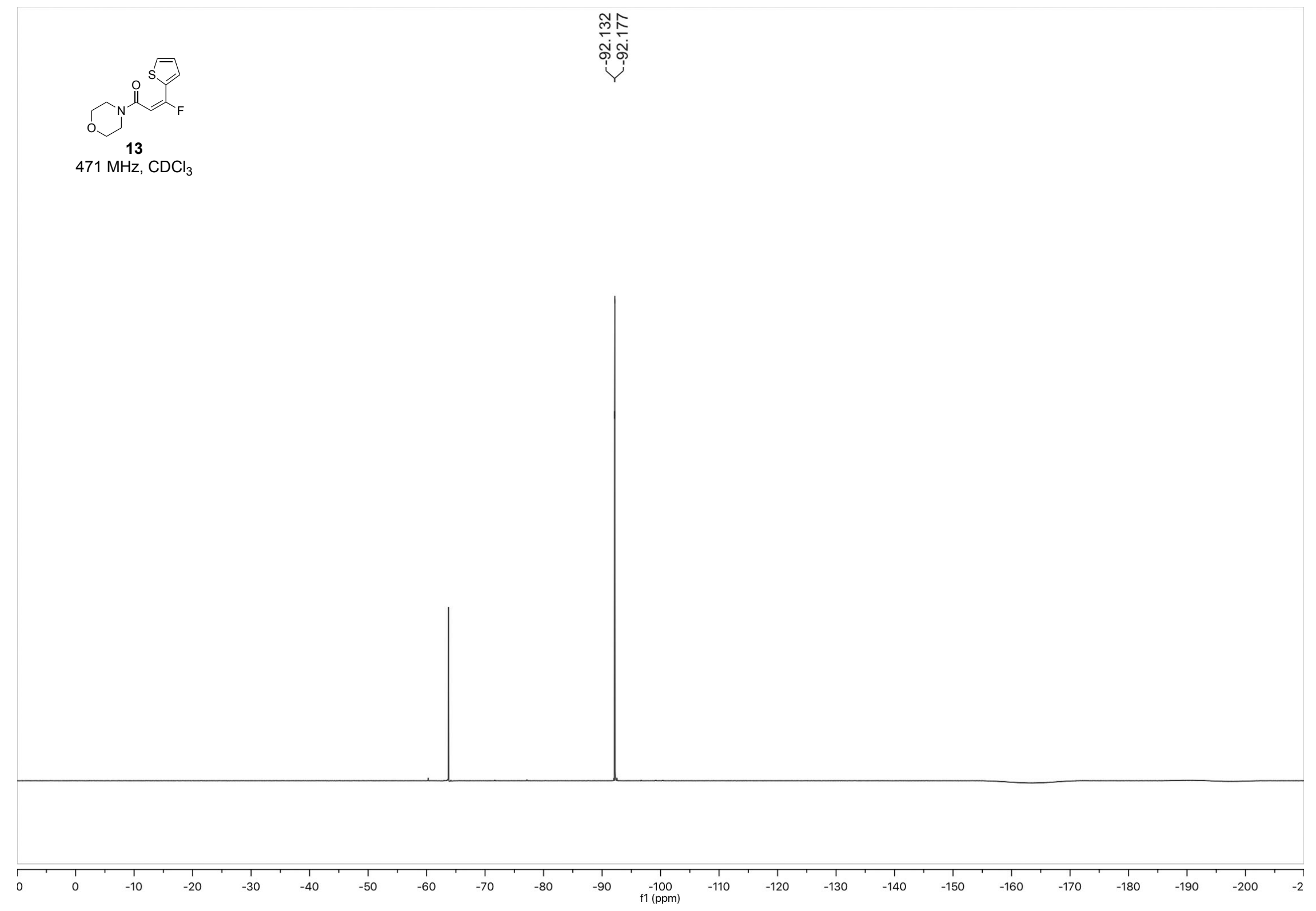




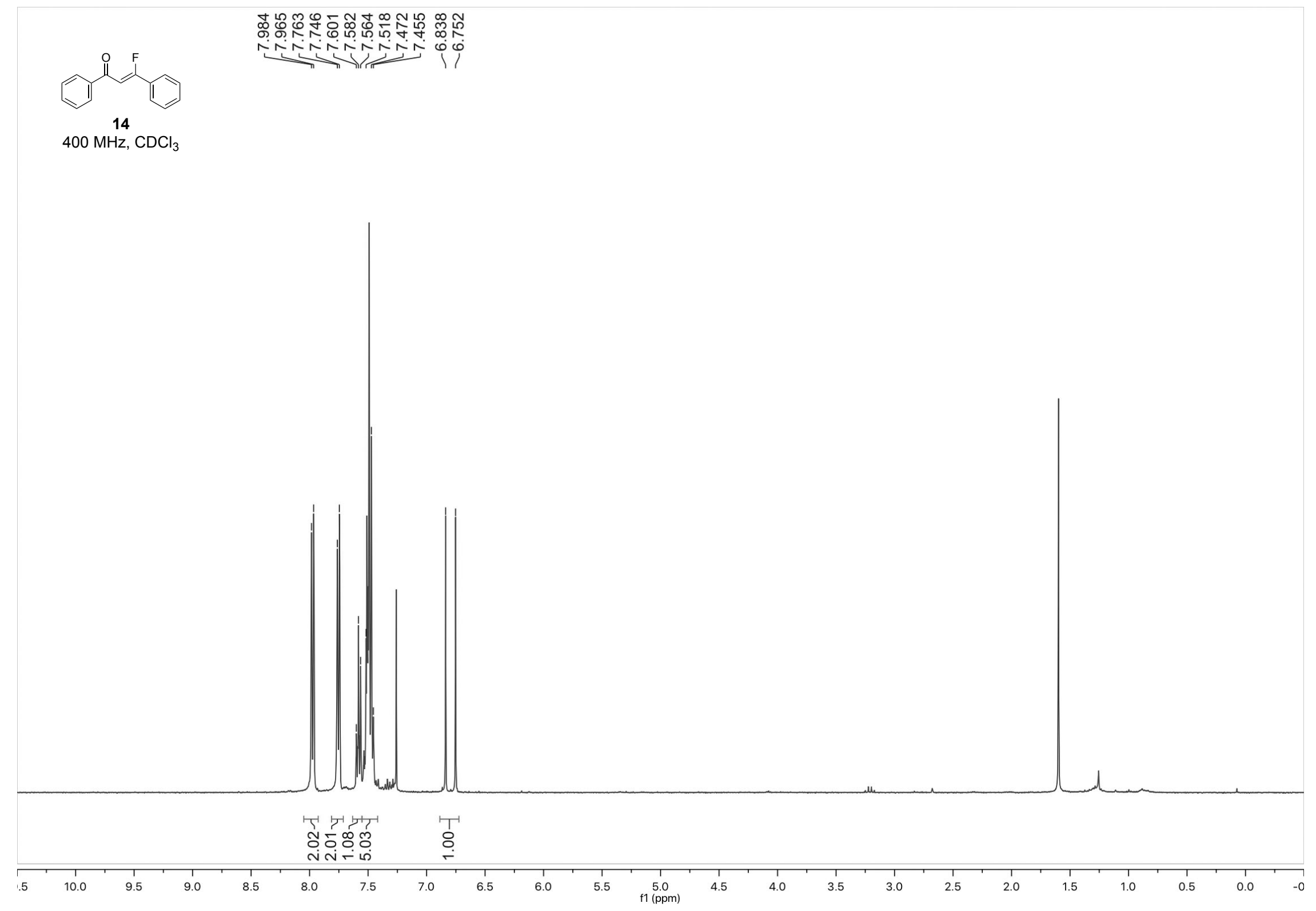




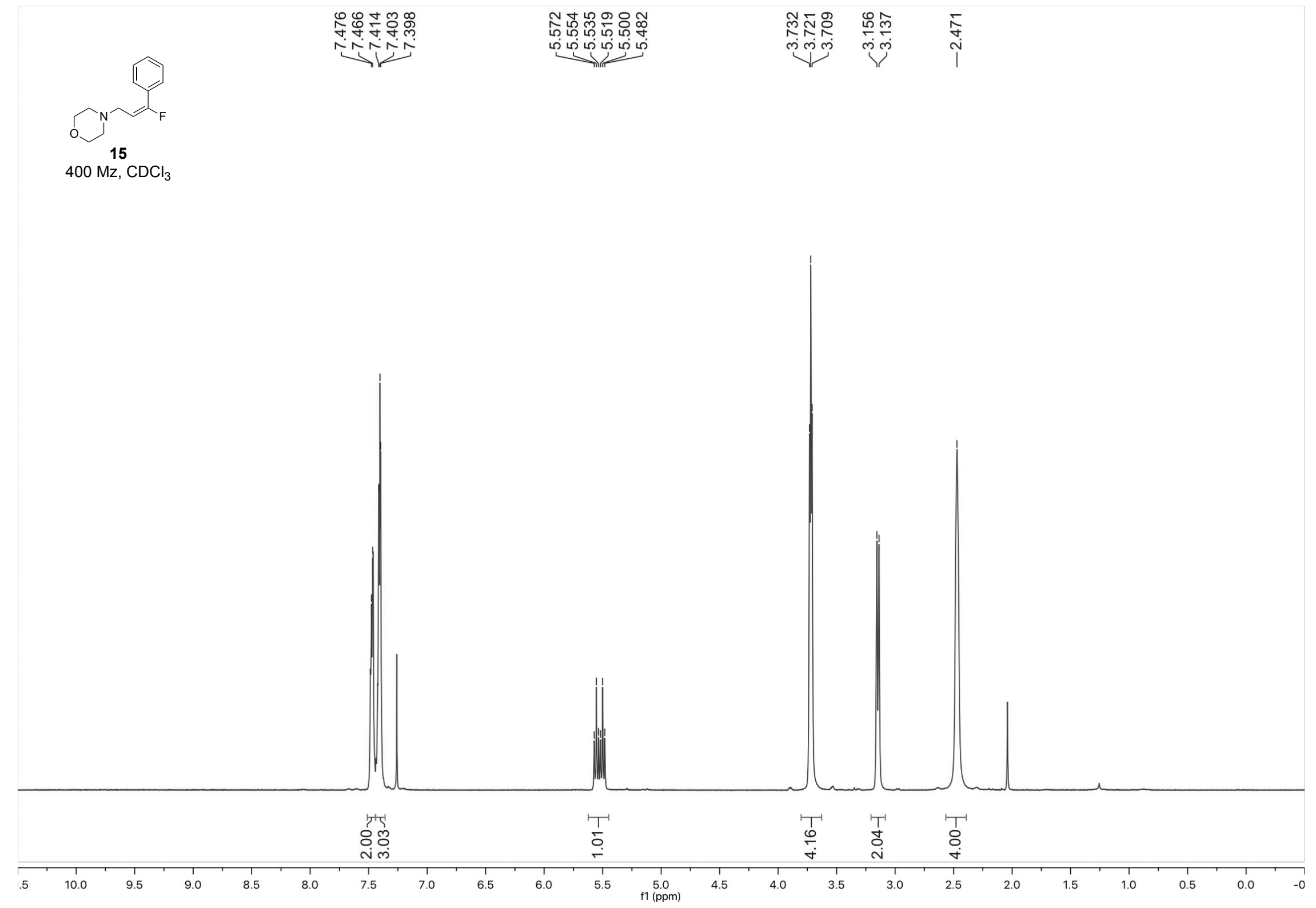



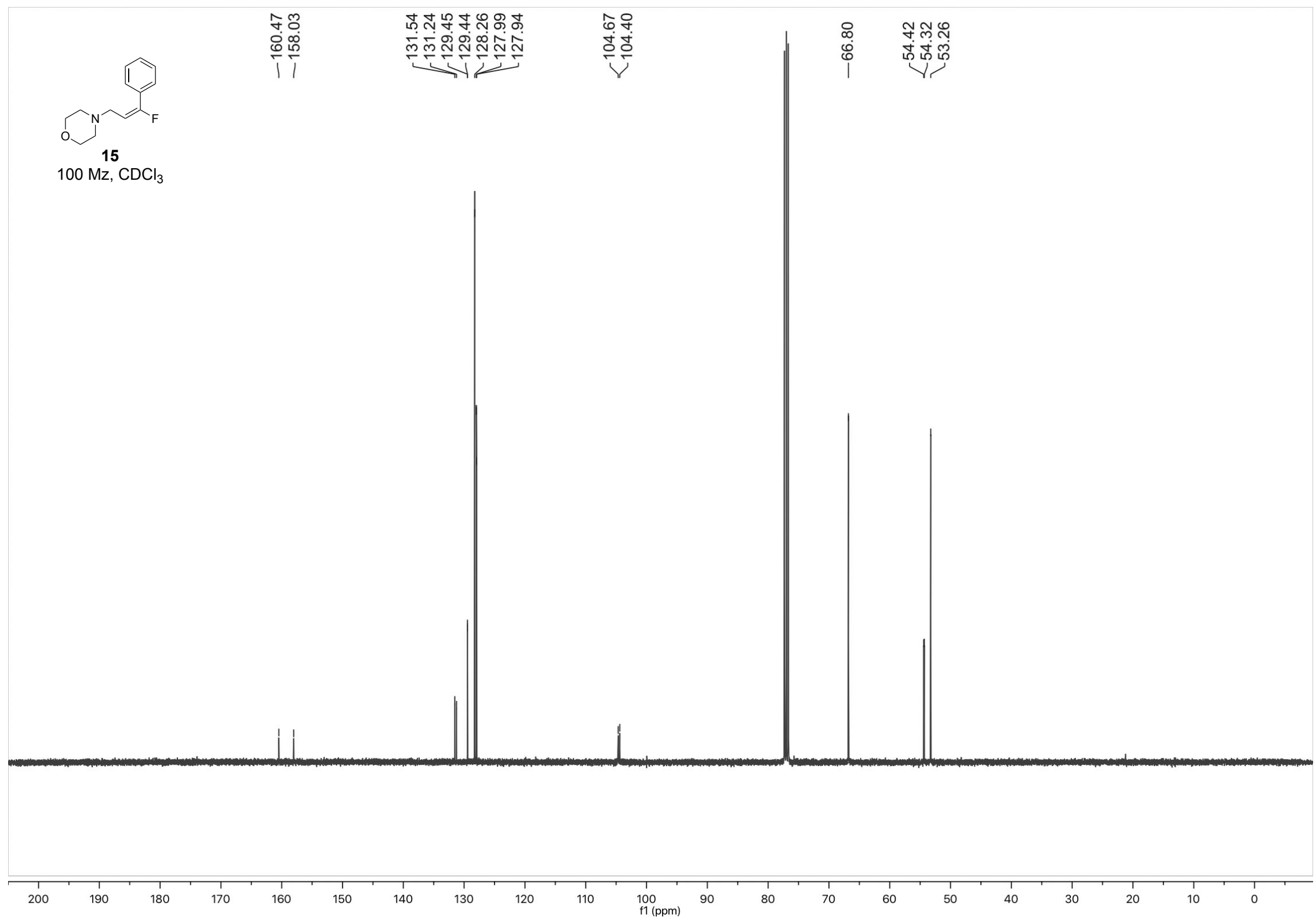


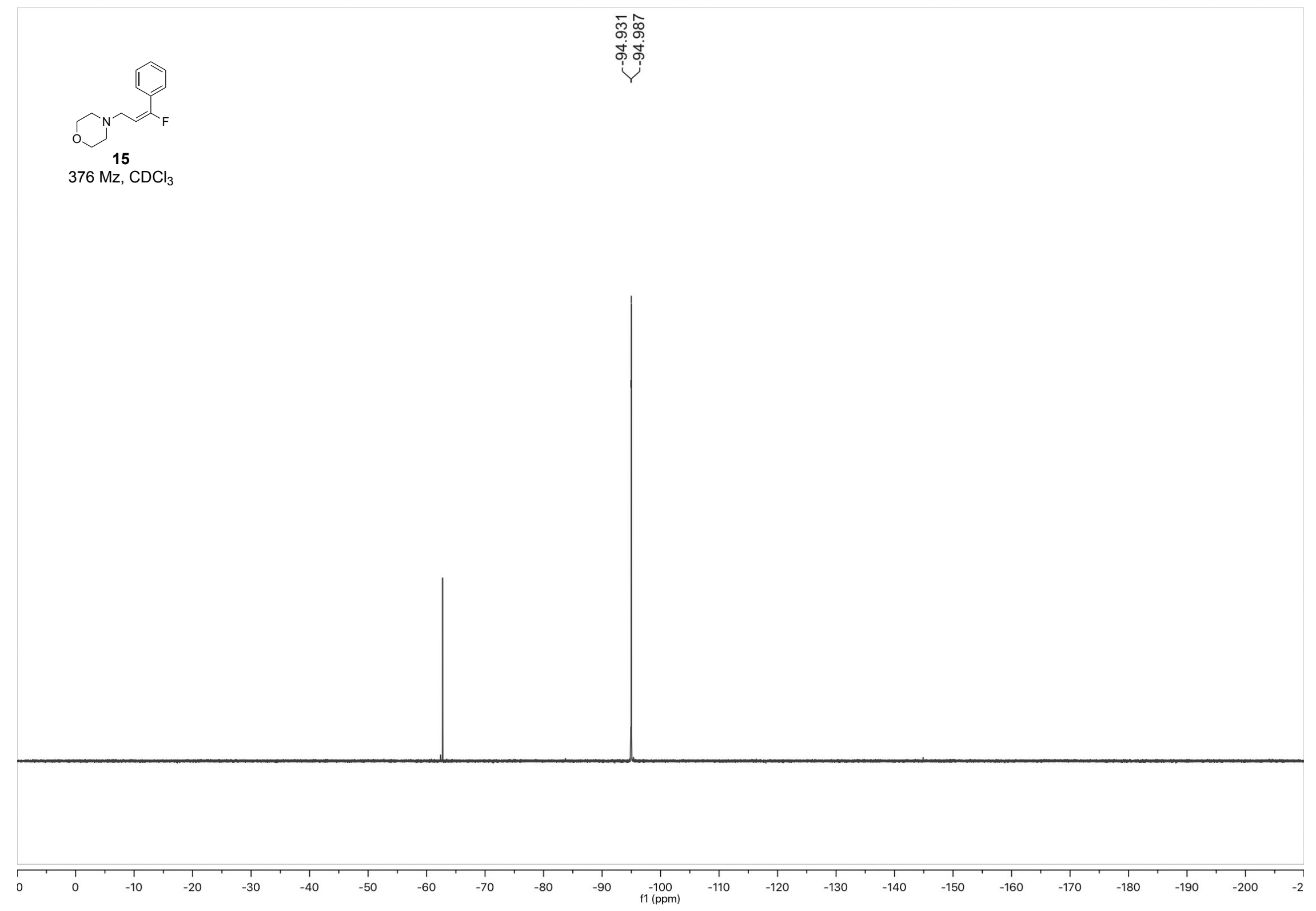




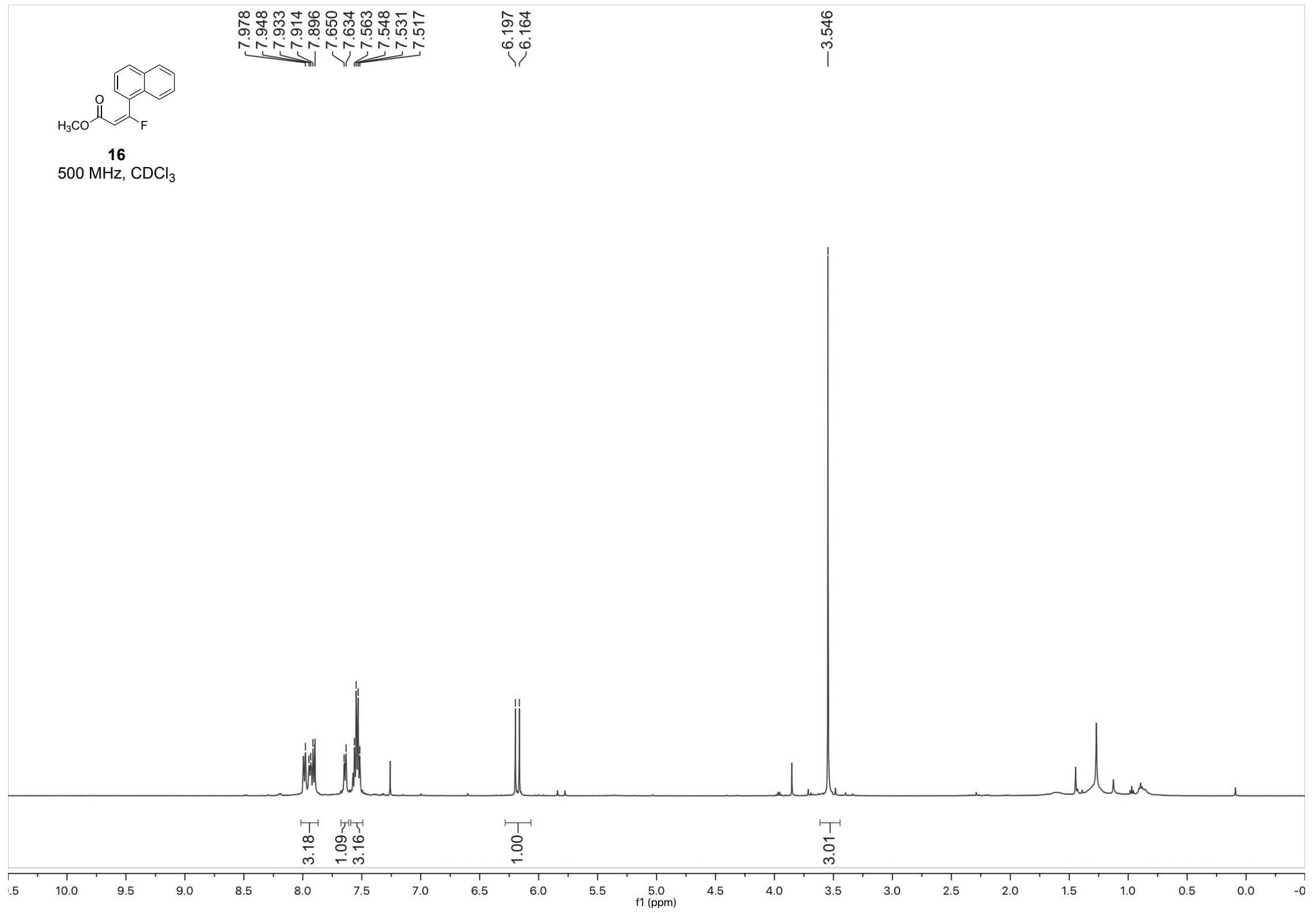




$$
11
$$




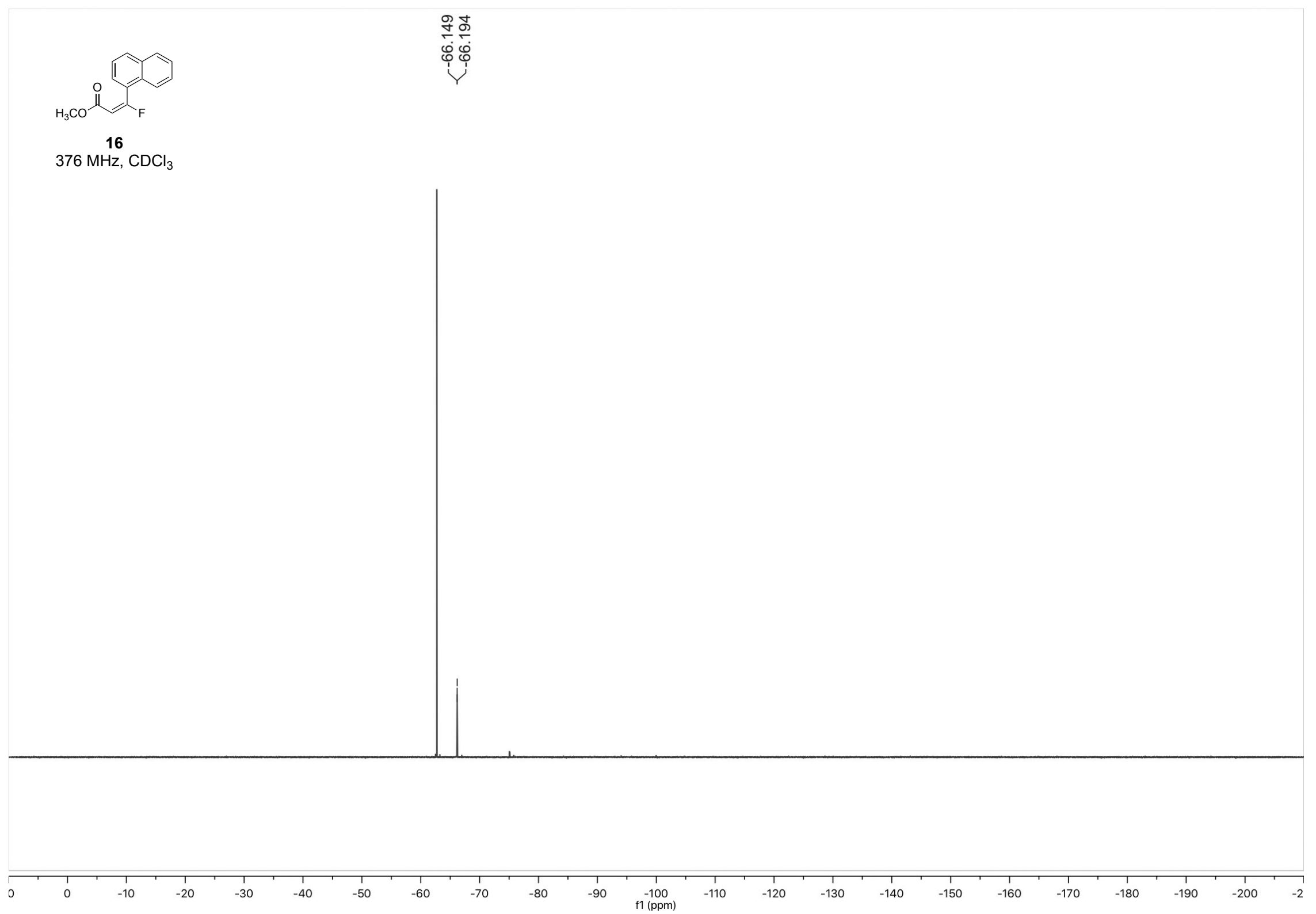

